\title{
Dynamic Zero-Covid strategy curtails mutagenesis and emergence of new variants of the SARS-CoV-2
}

Lunbiao Cui ( $\square$ lbcui@jscdc.cn )

Jiangsu Provincial Center for Disease Control and Prevention, Nanjing, P.R China

\section{Liguo Zhu}

Jiangsu Provincial Center for Disease Control and Prevention, Nanjing, P.R China

Jun Zhang

Yangzhou Center for Disease Control and Prevention, Yangzhou, P.R China

Huafeng Fan

Nanjing Municipal Center for Disease Control and Prevention, Nanjing, P.R China

Yongxiang Yi

Jun Zhao

The Third People's Hospital of Yangzhou, Yangzhou, P.R China

\section{Yiyue Ge}

Jiangsu Provincial Center for Disease Control and Prevention, Nanjing, P.R China

Shenjiao Wang

Jiangsu Provincial Center for Disease Control and Prevention, Nanjing, P.R China

\section{Xu Han}

Nanjing Medical University, Nanjing, China

\section{Xingsu Gao}

Nanjing Medical University, Nanjing, China

\section{Xian Qi}

Jiangsu Provincial Center for Disease Control and Prevention, Nanjing, P.R China

Jianguang Fu

Jiangsu Provincial Center for Disease Control and Prevention, Nanjing, P.R China

Zhifeng Li

Huan Fan

Jiangsu Provincial Center for Disease Control and Prevention, Nanjing, P.R China Huiyan Yu

Jiangsu Provincial Center for Disease Control and Prevention, Nanjing, P.R China

Fei Deng

Jiangsu Provincial Center for Disease Control and Prevention, Nanjing, P.R China

Kangchen Zhao

Jiangsu Provincial Center for Disease Control and Prevention, Nanjing, P.R China

Xiaojuan Zhu 
Jiangsu Provincial Center for Disease Control and Prevention, Nanjing, P.R China

Jianli Hu

Jiangsu Provincial Center for Disease Control and Prevention, Nanjing, P.R China

Jianjun Li

Jiangsu Provincial Center for Disease Control and Prevention, Nanjing, P.R China

\section{Jing Ai}

Jiangsu Provincial Center for Disease Control and Prevention, Nanjing, P.R China

\section{Guodong Kang}

Jiangsu Provincial Center for Disease Control and Prevention, Nanjing, P.R China

\section{Ya Shen}

Jiangsu Provincial Center for Disease Control and Prevention, Nanjing, P.R China

\section{Guangjie Jin}

Jiangsu Provincial Center for Disease Control and Prevention, Nanjing, P.R China

\section{Furu Wang}

Jiangsu Provincial Center for Disease Control and Prevention, Nanjing, P.R China

\section{Zhong Zhang}

Nanjing Municipal Center for Disease Control and Prevention, Nanjing, P.R China

\section{Min $\mathrm{He}$}

Nanjing Municipal Center for Disease Control and Prevention, Nanjing, P.R China

\section{Songning Ding}

Nanjing Municipal Center for Disease Control and Prevention, Nanjing, P.R China

\section{Yin Wang}

Yangzhou Center for Disease Control and Prevention, Yangzhou, P.R China

\section{Yuying Dong}

Yangzhou Center for Disease Control and Prevention, Yangzhou, P.R China

\section{Yao Huang}

Yangzhou Center for Disease Control and Prevention, Yangzhou, P.R China

\section{Changhua Yi}

College of Veterinary Medicine, Qingdao Agricultural University

\section{Longyu Wang}

The Second Hospital of Nanjing, Nanjing, P.R China

\section{Yudong Jiao}

The Third People's Hospital of Yangzhou, Yangzhou, P.R China

\section{Jinfu Wang}

The Third People's Hospital of Yangzhou, Yangzhou, P.R China

\section{Jian Li}

Southeast University, Nanjing, P.R China

\section{Hongde Liu}

Southeast University 


\section{Jingxin Li}

Jiangsu Provincial Center for Disease Control and Prevention, Nanjing, P.R China

Changjun Bao

Jiangsu Provincial Center for Disease Control and Prevention

Ming Wu

Jiangsu Provincial Center for Disease Control and Prevention, Nanjing, P.R China

Fengcai Zhu

Jiangsu Provincial Center for Diseases Control and Prevention

\section{Biological Sciences - Article}

\section{Keywords:}

Posted Date: December 28th, 2021

DOI: https://doi.org/10.21203/rs.3.rs-1195945/v1

License: (c) (1) This work is licensed under a Creative Commons Attribution 4.0 International License.

Read Full License 


\title{
Dynamic Zero-Covid strategy curtails mutagenesis and emergence of new variants of the SARS-CoV-2
}

Lunbiao Cui ${ }^{16,7 * \dagger}$, Liguo $\mathrm{Zhu}^{1 \dagger}$, Jun Zhang ${ }^{2 \dagger}$, Huafeng $\mathrm{Fan}^{3 \dagger}$, Yongxiang $\mathrm{Yi}^{4 \dagger}$, Jun $\mathrm{Zhao}^{5 \dagger}$, Yiyue $\mathrm{Ge}^{1,6,7 \dagger}$, Shenjiao Wang ${ }^{1 \dagger}$, Xu Han ${ }^{7}$, Xingsu $\mathrm{Gao}^{7}$, Xian $\mathrm{Qi}^{1}$, Jianguang $\mathrm{Fu}^{1}$, Zhifeng $\mathrm{Li}^{1}$, Huan $\mathrm{Fan}^{1}$, Huiyan $\mathrm{Yu}^{1}$, Fei Deng ${ }^{1}$, Kangchen $\mathrm{Zhao}^{1}$, Xiaojuan $\mathrm{Zhu}^{1}$, Jianli $\mathrm{Hu}^{1}$, Jianjun $\mathrm{Li}^{1}$, Jing $\mathrm{Ai}^{1}$, Guodong Kang ${ }^{1}$, Ya Shen ${ }^{1}$, Guangjie Jin ${ }^{1}$, Furu Wang ${ }^{1}$, Zhong Zhang ${ }^{3}$, Min $\mathrm{He}^{3}$, Songning Ding ${ }^{3}$, Yin Wang ${ }^{2}$, Yuying Dong ${ }^{2}$, Yao Huang ${ }^{2}$, Changhua $\mathrm{Yi}^{4}$, Longyu Wang ${ }^{4}$, Yudong Jiao ${ }^{5}$, Jinfu Wang ${ }^{5}$, Jian $\mathrm{Li}^{8}$, Hongde Liu ${ }^{8}$, Jingxin $\mathrm{Li}^{1,6,7}$, Changjun $\mathrm{Bao}^{1}$, Ming Wu ${ }^{1 *}$, Fengcai Zhu ${ }^{1,6,7,9^{*}}$
Affiliations:
1. NHC Key laboratory of Enteric Pathogenic Microbiology, Jiangsu Provincial Center for Disease Control and Prevention, Nanjing, P.R China
2. Yangzhou Center for Disease Control and Prevention, Yangzhou, P.R China
3. Nanjing Municipal Center for Disease Control and Prevention, Nanjing, P.R China
4. Nanjing Infectious Diseases Clinical Medical Center (The Second Hospital of Nanjing, Nanjing University of Chinese Medicine), Nanjing, P.R China
5. The Third People's Hospital of Yangzhou, Yangzhou, P.R China
6. Institute of Global Health and Emergency Pharmacy, China Pharmaceutical University, Nanjing, P.R China
7. Center for Global Health, School of Public Health, Nanjing Medical University, Nanjing, China
8. Southeast University, Nanjing, P.R China
9. Shanghai Institute of Infectious Disease and Biosecurity, Shanghai, P.R China.

$\dagger$ These authors contributed equally: Lunbiao Cui, Liguo Zhu, Jun Zhang, Huafeng Fan, Yongxiang Yi, Jun Zhao, Yiyue Ge, Shenjiao Wang.

* A list of corresponding authors. Email: lbcui@jscdc.cn, jswuming@ @ip.sina.com. jszfc@vip.sina.com.

\begin{abstract}
Within the local outbreak period of SARS-CoV-2 Delta variant in Nanjing and Yangzhou, China, we analyzed the mutation process of the Delta variants in 520 cases, as well as the production, spread and elimination of new mutant strains under the non-pharmaceutical interventions (NPI) strategy. The investigation on distribution of COVID-19 cases and phylogenetic analysis of SARS-CoV-2 genome sequences attributed to tracking the transmission chains, transmission chains were terminated by the isolation of the COVID-19 patients and quarantine of close-contracts, suggesting the importance of NPI in prompting some mutations to disappear and stopping the transmission of new variants. Dynamic zero-Covid strategy has been implemented successfully to against the second-largest local epidemic caused by an imported COVID-19 case in China.
\end{abstract}


After almost two years, the coronavirus disease 2019 (COVID-19) pandemic continues to sweep across the globe. With the Delta variants dominating, the world is facing challenges to address the public health emergency. The severe acute respiratory syndrome coronavirus 2 ( SARS-CoV-2) is prone to mutations like other RNA viruses. Genome sequencing of SARS-CoV-2 has shown a nucleotide substitution rate of $\sim 1 \times 10^{-3}$ per year ${ }^{1}$. As of 3 December 2021, 3,245,521 variants have been identified from 5,893,087 SARS-CoV-2 sequences, where mutations have been mainly observed in the open reading frame (ORF) and spike (S) protein regions ${ }^{2}$. Such changes in the genome may affect virus' properties such as pathogenicity, infectivity, and alter the effectiveness of vaccines, therapeutic medicines, and diagnostic tools 3. The World Health Organization (WHO) has defined Variants of Concern (VOCs) and Variants of Interest (VOIs) based on their characteristics. A total of 5 VOCs (Alpha, Beta, Gamma, Delta and Omicron) and 2 VOIs (Lambda and Mu) have been described ${ }^{4}$. Currently, out of 839,119 genomes sequenced from the virus samples collected in the last 60 days, $837,253(99.8 \%)$ belong to the Delta variant, $314(<0.1 \%)$ to Gamma, $160(<0.1 \%)$ to Alpha, $159(<0.1 \%)$ to Omicron, $14(<0.1 \%)$ to Beta, and $<0.1 \%$ comprised other circulating variants (including VOIs Mu and Lambda). These statistics suggest that the Delta variants have become the dominant variants ${ }^{5}$. Concerningly, several studies have shown that the infectivity and viral loading caused by the Delta variants are increased when compared to wild type strains and other VOCs. ${ }^{6-8}$

The evolution of viruses is usually influenced by random mutations and the selective pressure exerted by drugs or immune responses elicited against the virus ${ }^{9}$. However, social control policies, such as measures of eradication and elimination, have profound influence on the course of epidemics by stopping or retarding the pace of viral natural evolution ${ }^{10}$. The zeroCovid strategy means eliminating all cases of SARS-CoV-2 from a country or region, which is different from eradication that means completely eradicating the source of the infectious disease. The eradication of infectious diseases has been achieved only once in history, with smallpox, a momentous achievement for global public health. Following intensive vaccination campaigns, in 1980, the 33rd World Health Assembly officially declared the world free from smallpox. Similarly, since 1988, significant effort has been put into the elimination of poliomyelitis viruses. Consequently, infections caused by the wild type (WT) poliovirus have decreased by over $99 \%$. Of the 3 strains of WT poliovirus (type 1, type 2, and type 3), WT poliovirus type 2 was eradicated in 1999 and no cases of WT poliovirus type 3 have been reported since the last reported case in Nigeria in November 2012. This success has been largely accomplished by strengthening routine immunization, catch-up immunization and high-quality of AFP surveillance ${ }^{11,12}$. Despite this success, complete eradication polio has been out of reach ${ }^{13-17}$. On 2021, WHO officially certified China as malaria-free after the country reported no indigenous cases of the disease for 4 consecutive years, following application of artemisinin, indoor residual spraying, roll-out insecticide-treated bed-nets, coupled with a 1:3:7 surveillance and response system ${ }^{18}$. Leprosy was eliminated through the non-pharmaceutical interventions (NPI) and pharmaceutical interventions (PI). Plague and Ebola have achieved elimination through international quarantine policies. Among the emerging infectious diseases, Nipah virus, Henry virus and SARS virus have been basically eradicated through strict NPI measures. These experiences in eradication and elimination of diseases provide effective and referential ideas for the prevention and control of COVID-19.

NPI (to discover and isolate the source of infection) strategy was implemented during the epidemic prevention and control process in Wuhan, China. Remarkably, this strategy yielded 
zero-covid cases in 76 days. In fact, while China has implemented the zero-Covid strategy successfully, attracting global attention, South Korea, New Zealand, Taiwan, Hong Kong, Macao, Australia along with other countries or regions are also working hard to implement the zero-Covid strategy. In the second-largest local epidemic caused by an imported COVID-19 case in Nanjing and Yangzhou, China, following the first-largest local epidemic in Wuhan, we systematically dissected the mutation process of the Delta variants in different individuals, as well as the production, spread and elimination of new mutant strains under the intervention of the NPI strategy.

\section{Source and local spread of SARS-CoV-2 Variants}

On July 10, 2021, a total of 301 passengers flying on the Boeing 777-300ER flight from Moscow, Russia arrived at Nanjing Lukou International Airport (NKG). When tested for COVID-19 at the time of entry using the SARS-CoV-2 RT-PCR test, five COVID-19 positive cases were detected amongst the arrivals. Subsequent genome analysis revealed that the passengers were infected with the Delta variants and one of the cases (Nanjing/Yu) carried very high loadings of the Delta variants $(\mathrm{Ct}=14.5)$. Two COVID-19 cases were detected on the 5th and 7th day after arrival of the flight 777-300ER at NKG. However, the two cases are unlikely to be the source of infection that subsequently spread locally because they tested negative in the cabin. According to China's quarantine policy, all the arrivers are lodged in a hotel far from the NKG for quarantine and observed for 14 days immediately after disembarking from the plane. Therefore, the newly detected COVID-19 cases did not directly contact the infected COVID-19 cases among the arrivers. One hour after all the passengers and aircrew left the cabin and after prophylactic disinfection, 16 cleaning staff boarded the cabin to clean. Among them, 6 cases of COVID-19 were found during the weekly nucleic acid testing on July 20; five of them had a history of direct contact with the excreta or pollutants of the case-Nanjing/Yu, including two who cleaned the toilets which case Nanjing/Yu used, two cleaned the aisles where he walked through, and one cleaned up the garbage from Nanjing/Yu's seat. Although the cleaning staffs were wearing masks and gloves, the gloves did not cover the cuffs of the protective clothing. This may have caused their wrists to be exposed. Their hands were not disinfected during the process of cleaning, handling garbage and removing protective clothing after cleaning, and gloves were slipped off, which may have caused hand contamination. Through whole-genome sequencing analysis, it was found that they were infected with the Delta variants. The genomes of the virus isolated from the cleaning staff were highly homologous to those detected in case Nanjing/Yu, showing complete identity, including the spike H146Y mutation of SARS-CoV-2. No other COVID-19 cases from that flight had the spike H146Y mutation and showed low viral loadings. In addition, since July 1 to July 20, a total of six international flights arrived in Nanjing and some passengers have been found infected with SARS-CoV-2, but none of them carried the Delta variants. The outbreak among cleaning staff was not spatially related to the Guangdong and Yunnan outbreak, where local cases of Delta variants have been previously reported. Based on the epidemiological and genomic data, it can be inferred that the cleaning staff probably contracted COVID-19 through indirect contact with the case-Nanjing/Yu.

\section{Indigenous spread of SARS-CoV-2}


Nanjing is located on the East coast of China, with 9,314,685 permanent residents. From the onset of the outbreak of the epidemic at NKG on July 20th to September 9th, a total of 780 COVID-19 cases were reported in Nanjing and Yangzhou. In the Nanjing outbreak, the 10-day period between the cabin exposure and the discovery of the COVID-19 cases, the epidemic ran its natural course and was defined as "natural epidemic period, NE". From July 21 st to 26 th, nucleic acid tests were carried out for close contacts and high-risk populations. Furthermore, hotel quarantine and treatment in designated hospitals for COVID-19 cases, closure and control of the communities where the cases were located were carried out. These measures could not yet achieve complete hotel quarantine for close contacts, so this 6-day period was termed as "incompletely intervened period, II". Starting from July 27th and 9 days thereafter, nucleic acid tests for close contacts and high-risk populations were performed. Hotel quarantine and treatment in designated hospitals for cases, closure of the communities where the cases were located, and hotel quarantine of all close contacts were achieved. Therefore, this period was called "completely intervened period, CI". The benefits of implementation of vigorous intervention started becoming visible after CI, with the number of COVID-19 cases dropping from over 10 COVID-19 cases per day to less, and the last case was detected on August 11. This phase was called as "epidemic regression period, ER". During the NE, II, CI and ER period, the number of COVID-19 cases or nucleic acid positives found in Nanjing were 41, 109, 64 and 7, respectively (Fig. 1A).

Yangzhou is located about $100 \mathrm{kms}$ to the Northeast of Nanjing. The epidemic in Yangzhou started from accidental exposure to a COVID-19 case of Nanjing on July 19. The patient travelled to Yangzhou on July 21. She was confirmed as COVID-19 positive by RT-PCR on July 27 in Yangzhou and intervention measures were put in place on July 28. The NE period was 9 days and a total of 48 COVID-19 cases occurred during this time. Hereafter, during the 10-day II period, 8-day CI period and 10-day ER period, 358, 141, and 12 COVID-19 cases were detected, respectively (Fig. 1B).

Phylogenetic analysis revealed that all the genomes of viruses isolated from COVID-19 patients of Nanjing and Yangzhou fell into one clade. The viruses from Yangzhou COVID-19 cases belonged to the branch of those from Nanjing COVID-19 cases, which was consistent with the fact that the COVID-19 local outbreak in Yangzhou originated from the old lady case (YZ001) who travelled from Nanjing to Yangzhou. None of the genomes from Yangzhou COVID-19 cases fell into the majority of genomes from Nanjing COVID-19 cases may due to the timely lockdown measure of Yangzhou. The genomes from Nanjing and Yangzhou COVID-19 cases were not clustered with those of early genomes of imported COVID-19 cases detected before July 10, 2021 in Mainland, China and the early Delta variants DL-ILBS-22053 isolated in New Delhi, India. (Fig. 1C).

\section{Characteristics of mutations of the virus causing local epidemic}

Compared with Wuhan-Hu-1 strain, the Delta variants isolated from case Nanjing/Yu and the early infected local cases in Nanjing had 35 mutations in the nucleotide sequence and three deletion sites (Extended data Table 1). The mutations seem to have changed many amino acids. Among them, there were four amino acid (aa) substitutions in the nucleocapsid protein $(\mathrm{N})$, namely D63G, R203M, G215C and D377Y. There was one amino acid substitution, I82T, in the membrane protein $(\mathrm{M})$. There were 16 amino acid substitutions in other ORF regions, including 11 substitutions in ORF1ab (K261N, A1306S, P2046L, P2287S, V2930L, T3255I, 
T3646A, P4715L, G5063S, P5401L, A6319V), one substitution in ORF3a (S26L), three substitutions in ORF7a (P45L, V82A, T120I) and one substitution in ORF8 (ERF119-120DF) (Fig. 2A). G5063S (nucleotide G15451A) substitution in RdRp resulted in the mismatch of Charite primer, which may affect its detection efficiency because it mapped to the $3^{\prime}$ primer region. Another R203M (nucleotide acid G28881T) substitution in N gene may have no influence on efficiency of the primer designed by China center for Disease Control and Prevention (China CDC) since its location maps to the first nucleotide of the $5^{\prime}$ primer region (Fig. 2C). No amino acid substitution was observed in the envelope (E) protein, thus there is no effect on the diagnostic reagents targeting $E$ protein. In addition to the impact of mutations on testing, previous studies have shown that mutations in the ORF1ab region may increase the viral load and cell mortality ${ }^{19}$, and probably be related to the decrease of lymphocytes (CD3 ${ }^{+}$ $\mathrm{T}, \mathrm{CD} 4^{+} \mathrm{T}, \mathrm{CD} 8^{+} \mathrm{T}, \mathrm{CD} 19^{+} \mathrm{B}$ cells and $\mathrm{CD} 16^{+} \mathrm{CD} 56^{+} \mathrm{NK}$ cells) and cytokine release (IL-6 and IL-8) ${ }^{20}$. ORF3a mutations are probably likely to impact the viral life cycle, virulence, infectivity, ion channel formation, and virus release ${ }^{21}$. Furthermore, the deletion in ORF8 may reduce the virulence of SARS-CoV-2, resulting in harmless mild infections ${ }^{22}$. Moreover, SARS-CoV-2 hijacks the host ubiquitin system to enhance ORF7a's ability to antagonize IFNI responses. Therefore, mutations in ORF7a would change the IFN-I signaling and affect viral mechanisms responsible for suppressing the immune response ${ }^{23,24}$. Another study suggested that I82T substitution in M protein could affect the putative glucose transport transmembrane helices, leading to immune evasion ${ }^{25}$.

The S protein of SARS-CoV-2 plays an important role in the infection process and therefore has received extensive attention. Among the 38 variations at different sites identified by comparing the genomes, eight amino acid substitution sites and one amino acid deletion are located in the S protein (Fig. 2B, Extended data Table 1). These include T19R, G142D, H146Y, L452R, T478K, D614G, P681R, D950N substitutions and the deletion of 156-157DEL. Within these, five amino acid substitutions (G142D, H146Y, D614G, L452R and T478K) and one amino acid deletion (157DEL) may be related to the reduced binding ability of existing neutralizing antibodies (Extended data Table 2) ${ }^{26-34}$. The P681R is located at the cleavage site of the furin protease, which makes the cleavage more efficient and the virus enters the host cell faster. It is also known to enhance the replication of virus, resulting in higher levels of viral loading and increased transmission ${ }^{35-37}$. It is worth mentioning that, the H146Y mutation in the S protein may further exacerbate the effects of vaccines and antibody-based interventions as predicted by modeling and results observed in the real world. According to the results of our previously published studies, the H146 site of NTD in SARS-CoV-2 S protein is an important neutralization site, which has strong binding activity with neutralizing antibodies like $\mathrm{FC} 0{ }^{28}$. The neutralizing antibody (4A8) reported by Wei Chen et. al. also showed similar properties ${ }^{29}$. According to the GISAID database, Delta variants containing the $\mathrm{S}$ protein H146Y mutation were reported from 8 countries, including Russia, before July 10th ${ }^{38}$.

\section{Mutations of SARS-CoV-2 under dynamic zero-Covid strategy}

Several transmission chains were formed in the local epidemic caused by the Delta variants where a wide range of single nucleotide variations (SNV) were observed. These included the 35 basic mutation (nucleotide mutation, including H146Y mutation), 35 basic \& $\mathrm{n}$ iSNV (rare) mutation ( $\mathrm{n} \geq 1$ ), 35 basic + C346T mutation, 35 basic + C346T \& $\mathrm{n}$ iSNV (rare) mutation ( $\mathrm{n} \geq 1$ ), 35 basic $+\mathrm{T} 2803 \mathrm{C}$ mutation, 35 basic $+\mathrm{T} 2803 \mathrm{C} \& \mathrm{n}$ iSNV (rare) mutation $(\mathrm{n} \geq 1), 35$ 

$(\mathrm{n} \geq 1$ ), respectively. (Fig. 3)

Among the 221 COVID-19 cases in Nanjing, whole-genomic sequences of 167 cases were successfully compiled. Sequence analysis revealed that a total of $48 \mathrm{SNV}$ combinations were formed, mainly including C346T or T2803C homozygous mutations, and other rare homozygous mutations of the C346T or T2803C type (Fig. 3A, Extended data Table 3 and Table 5). The number of SNV combinations formed in NE, II, CI, and ER periods were 16, 17, 13, and 2, respectively (Fig. 3C). Among these 48 SNV combinations, 39 were terminated in complete hotel quarantine, and 9 were spontaneously terminated. The spontaneously terminated cases had the opportunity to infect others. Thus, about $81.25 \%$ (39/48) of mutations were terminated by hotel quarantine measures. Specifically, 3, 17, 15 and 4 SNV combinations were terminated with NPIs in NE, II, CI, and ER periods, while 2, 5 and 2 SNV combinations were self-terminated in NE, II and CI periods, respectively. In addition, several SNV combinations emerged in single cases. As shown in Fig.3C, 5, 14, 8 and 1 SNV combinations terminated in NE, II, CI, and ER periods, of which 3, 8, 7 and 1 were terminated by hotel quarantine measures, and others were self-terminated in the four periods, respectively.

Among the 559 COVID-19 cases in Yangzhou, 353 whole-genome sequences were obtained and 89 SNV combinations were identified (Extended data Table 4 and Table 6). Based on epidemiological evidence, the unique original case in Yangzhou was an old lady YZ001, with T2803C mutation add to the 38 basic nucleotide mutations in Nanjing, secondary transmission from NJ047, who was firstly observed with the T2803C substitution during the early II' period in Nanjing, so all cases had 36 basic mutations in Yangzhou. Sequence analysis revealed that T7513C homozygous mutations were added, and other rare homozygous mutations with T7513C or T2803C type (Fig. 3B). The number of SNV combinations formed in NE, II, CI and ER periods were 15, 44, 28, and 2, respectively. Of the 89 SNV combinations, 53 were terminated in complete hotel quarantine, and 36 were self-terminated. The self-terminated cases had the chance to infect others. The mutations terminated due to hotel quarantine accounted for 59.55\% (53/89) (Fig. 3D) of the total cases, which was lower than that of Nanjing. This discrepancy could be attributed to the longer II period. Specifically, 2, 21 and 30 SNV combinations were terminated with NPIs in NE, II and CI periods, while 6, 15, 12 and 3 were self-terminated in NE, II, CI and ER periods, respectively. Several SNV combinations were also found in single cases in Yangzhou (Fig.3D), 7, 28, 22 and 2 SNV combinations terminated in NE, II, CI, and ER periods, of which 2, 16, 13 and 0 were terminated by hotel quarantine measures, and others were self-terminated in the four periods, respectively.

In this epidemic, the elimination of the Delta variants included two aspects. On one hand, the self-elimination of the Delta variants could terminate the transmission chain in the absence of NPI, the percentage of mutants eliminated in self-termination accounted for $32.85 \%$ of the total SNV combinations. On the other hand, the number of the SNV combinations decreased gradually from II to ER periods, and eventually the transmission chains were fully terminated, suggesting that hotel quarantine could effectively prompt some mutations to disappear and was important to stop the SNV combination of Delta variants in local epidemic.

Respiratory samples were collected from three COVID-19 cases at different time points to analyze the dynamics of the allele frequency in intra-host single nucleotide variations (iSNVs). Changes in the profiles of alternative allele frequencies (AAFs) were noted for samples collected at three different dates. Four iSNVs including T12175A, T16392C, G24410A (YZ409), and G28916T (YZ404) showed an increase in frequency and remained stable during 
second and third sampling. Two iSNVs including C23604G and C27527T (YZ409 and YZ404) showed high AAFs during first two sampling dates which decreased in third sampling date. Three iSNVs including G21987A, C27527T and G28916T (YZ018) displayed decreased AAFs during the second sampling date while having high AAFs in first and third sampling dates. Noteworthily, we observed some minor AAFs such as C7764T spontaneously disappear within the host, while T7513C (YZ018) continuously evolve and become homozygous at third sampling date (Fig. S1). T7513C substitution was a common SNP that emerged in more than 89 cases of Yangzhou epidemic. Quarantine of COVID-19 patients with YZ018 profile further confirmed zero-Covid strategy would help to stop the spread of the mutation and terminate the further evolution of the virus.

\section{Impact of the mutations on the epidemiology of COVID-19}

We analyzed the whole-genome sequence data obtained from 520 cases, which included 371 non-terminated cases and 149 terminated cases by multivariate logistic regression model. Only onset period of cases in the epidemic, COVID-19 vaccination state (Vaccined $\geq 30$ days) and clinical symptoms (Severe+Critical) were associated with termination of SNV combinations, while age, sex, Ct Value (viral loading), clustering and termination codon were not associated with termination of SNV combinations (Extended data Table 7). The termination rates of the last cases were higher in II and CI periods compared to NE and ER periods. Vaccinated cases ( $\geq 30$ days) were 2.33 times more likely to have their SNV combinations terminated than those who were not vaccinated, which could be related to the presence and known consequences of E484K/Q substitution, in spite of H146Y substitution in S protein region in the local Delta variants ${ }^{36}$.

In the local epidemic, based on whole-genome sequence analysis, G28209T located in ORF8b region results in a stop codon, terminating the synthesis of the peptide chain. The G28209T mutation was noted in several transmission chains among 8 cases in Nanjing, including 2 cases in 35 basic + C346T \& 1 iSNV mutation chain and 6 cases in 35 basic + C346T \& niSNV (rare) mutation chain. Four of them were the last COVID-19 cases, of which three were terminated by NPI and only one was stopped by self-termination, indicating the important role of NPI in stopping the transmission of variants with G28209T mutation. In previous studies, ORF8b protein of SARS-CoV-2 could trigger the activation of the NLRP3 inflammasome in macrophages in vitro and mediate release of interleukin-1 family cytokines. Therefore, an intense inflammasome formation characterizes the lungs of patients with fatal COVID-19 disease due to pneumonia and acute respiratory distress syndrome (ARDS) ${ }^{39}$. Published studies have indicated that the deletion of 382 nucleotides in the ORF8 region of the SARSCoV-2 variants could result in less systemic release of pro-inflammatory cytokines and milder infections, while the patient's symptoms last longer ${ }^{22}$. Another study found a mutation (Q27stop) that truncated the ORF8 region in the VOC-202012/01 variant, the deletion in ORF8 changed the clinical onset of the disease, which may mediate immune escape and affect the transmission of SARS-CoV-2 variants ${ }^{40}$.

Throughout this local epidemic, 81 amino acid mutations were found in different regions of SARS-CoV-2 among all cases, including 1, 3, 5, 11, 53, 2, 3 and 1 in E, M, N, S, ORF1ab, 
ORF3a, ORF7a and ORF10 domains respectively. Another 2 additional mutations (nucleotide A27010C and A5289T) have not been defined in previous sequences. Eleven novel mutations in the S protein like L5F, P384S, N542K, D574Y, A647S, H655Y, G769V, V951L, Q1005H, T1117I, and Q1208H are worthy of attention. Among these, L5F has been found in several variants around the world, especially in B.1.526, which may be related to the increased transmissibility of the variant strain. This probably resulted in the second wave of COVID-19 cases to extend its duration in New York in early $2021^{41}$. H655Y mutation emerging in B.1.1.529 variants and P.1 variants has been proven to evade neutralization by human monoclonal antibodies, and can spread rapidly in cats and persists at intermediate frequencies 4,42. In relation to the local epidemic, H655Y mutation was found among 6 sporadic cases in Yangzhou during the II and CI periods (from Aug 2nd-10th) and none subsequently cases, suggesting that the transmission of variants with this mutation may be terminated by the NPI measures. The G769V mutation was found in the R.1 lineage of SARS-CoV-2 in Japan. This mutation is not considered to be related to immune escape variants like the simultaneous E484K and W152L mutations, and its role needs to be further investigated ${ }^{43}$. T1117I mutation in the spike was found during the pandemic wave beginning in May 2020 in Costa Rica, whose frequency reached $29.2 \%$ in full genome analyses. Structural modeling of the T1117I mutation suggests a potential effect on the viral oligomerization needed for cell infection, but no differences with other genomes on transmissibility, severity nor vaccine effectiveness are predicted for this mutation ${ }^{44}$. In terms of the other 7 mutations, which were firstly identified and their information uploaded in GISAID database, the information of mutated locus and the corresponding biological changes in the function have not been reported. As can be analyzed from the chemical structure, the Q1005H and Q1208H substitution may abrogate intraprotomer hydrogen bond (H-bond) between the T716 side-chain and main-chain carbonyls of Q1005 and Q1208, but could form a symmetric histidine triad near the base of the spike. In addition, water-mediated interactions could promote the formation of hydrogen bonding between the histidines ${ }^{45}$. In terms of A647S mutation, after the hydrophobic and non-polar alanine converted to hydrophilic and polar serine, the hydroxyl group contained in serine may bind to the oligosaccharide chain of T645 and would lead to the changes of spatial structure between antigens and cells. Furthermore, the D574Y mutation would also be affected by glycan chains of T572 and T573 and may mediate immune escape, although its specific effects have not been confirmed in predicted models and the real world.

\section{Discussion}

At present, the Delta variants have become dominant worldwide. Countries that have significantly reduced the COVID-19 cases through vaccination have once again experienced an increase in cases; the rate of increase in cases being much faster than before. Immune escape of variants, leading to the immune protection obtained from vaccines and natural infections becoming ineffective, remains the biggest nightmare of mankind. From this perspective, the highly infectious Delta variants squeezing out the Beta variants with strong immune escape activity seem to help humans escape a catastrophe. Lessons learned from the successful control of local epidemics in Nanjing and Yangzhou, suggesting a key role for the analysis of mutations for case tracing and epidemic control, which prevented the formation of a dominant mutation due to the success of NPI measures that cleared COVID-19 cases. Many global teams are working to prevent and control the continuous variation observed in viruses. In fact, some of the models established based on bioinformatics and structural prediction could predict the 
location and nature of possible mutations in future, and the prediction results were confirmed later in the strains that emerged later in the course of the epidemic ${ }^{46-48}$. Finally, admittedly, there are some limitations in this research. The impact of the amino acid mutations on the spike conformation have not been verified by cryo- electron microscopy (EM) in the current study, and the impact of the mutations on glycosylation remains to be analyzed. However, as these mutations have not been discovered in previous studies and the variants had been wiped out, and the local epidemic is not significantly different from previous reports. In addition, glycosylation analysis requires a large number of viruses and individual differences in glycosylation between hosts are the limitations of the method itself. Therefore, the mechanism of these mutation sites is planned to be investigated in our future researches and will be shown in another complete report.

As long as the virus continues to spread, it will replicate and produce new mutation sites. Only extensive vaccination and strict public health management are the best means to reduce risks of contracting COVID-19. The local mutation patterns discovered in Nanjing and Yangzhou, as well as the effective preventive and control measures adopted, have greatly reduced the development of global mutations and made outstanding contributions to the development of global public health and the reduction of disease burden. It would also provide reference methods and ideas for the prevention and control of new outbreaks in the future.

\section{Online content}

Any methods, statements of data and code availability, acknowledgements, details of author contributions, competing interests and References, and extended data are available at supplementary materials.

\section{References and Notes}

1 Duchene, S. et al. Temporal signal and the phylodynamic threshold of SARS-CoV-2. Virus evolution 6, veaa061, doi:10.1093/ve/veaa061 (2020).

2 Center., N. G. D. Released Genome Sequences. https://ngdc.cncb.ac.cn/ncov/release genome?lang=en (2021).

3 Administration, U. S. F. a. D. Removal Lists of Tests That Should No Longer Be Used and/or Distributed for COVID-19: FAQs on Testing for SARS-CoV-2. https://www.fda.gov/medical-devices/coronavirus-covid-19-and-medicaldevices/removal-lists-tests-should-no-longer-be-used-andor-distributed-covid-19-faqstesting-sars-cov-2 (2021).

4 WHO. Tracking SARS-CoV-2 variants. https://www.who.int/en/activities/tracking-SARSCoV-2-variants/ (2021).

5 WHO. Weekly epidemiological update on COVID-19 - 30 November 2021. https://www.who.int/publications/m/item/weekly-epidemiological-update-on-covid-19--30-november-2021 (2021).

6 Prevention, C. f. D. C. a. What You Need to Know about Variants. https://www.cdc.gov/coronavirus/2019-ncov/variants/variant.html (2021).

7 Normile, D. Can 'zero COVID' countries continue to keep the virus at bay once they reopen? Science doi: 10.1126/science.acx9109 (2021).

8 Brown, C. M. et al. Outbreak of SARS-CoV-2 Infections, Including COVID-19 Vaccine 
Breakthrough Infections, Associated with Large Public Gatherings - Barnstable County, Massachusetts, July 2021. MMWR. Morbidity and mortality weekly report 70, 1059-1062, doi:10.15585/mmwr.mm7031e2 (2021).

9 Lauring, A. S. \& Andino, R. Quasispecies theory and the behavior of RNA viruses. PLoS pathogens 6, e1001005, doi:10.1371/journal.ppat.1001005 (2010).

10 GISAID. Genomic epidemiology of novel coronavirus - Global subsampling. https://nextstrain.org/ncov/gisaid/global (2021).

11 Yu, W. Z. et al. Poliomyelitis eradication in China: 1953-2012. The Journal of infectious diseases 210 Suppl 1, S268-274, doi:10.1093/infdis/jit332 (2014).

12 Wiysonge, C. S., Njamnshi, A. K., Nomo, E. \& Shey, M. S. Eradication of poliomyelitis. Lancet (London, England) 366, 1163-1164, doi:10.1016/s0140-6736(05)67477-6 (2005).

13 Okwo-Bele, J. M. \& Cherian, T. The expanded programme on immunization: a lasting legacy of smallpox eradication. Vaccine 29 Suppl 4, D74-79, doi:10.1016/j.vaccine.2012.01.080 (2011).

14 Henderson, D. A. Lessons from the eradication campaigns. Vaccine 17 Suppl 3, S53-55, doi:10.1016/s0264-410x(99)00293-5 (1999).

15 Global eradication of smallpox: WHO Global Commission for the Certification of Smallpox Eradication. Journal of the Medical Association of Thailand $=$ Chotmaihet thangphaet 62, 461 (1979).

16 Omata, K., Hashimoto, M., Watanabe, Y., Umegaki, T. \& Yamada, M. J. S. A. E. Report of the global commission for the certification of smallpox eradication. 62, 245-246 (1980).

17 Hall \& A. \%J Geneva, S., WHO,. The Global Eradication of Smallpox. Final Report of the Global Commission for the Certification of Smallpox Eradication. 58, 1-1 (1982).

18 Burki, T. Triumph in China as it is certified malaria-free by WHO. The Lancet. Infectious diseases 21, 1220-1221, doi:10.1016/s1473-3099(21)00491-6 (2021).

19 Yao, H. et al. Patient-derived SARS-CoV-2 mutations impact viral replication dynamics and infectivity in vitro and with clinical implications in vivo. Cell discovery 6, 76, doi:10.1038/s41421-020-00226-1 (2020).

20 Zhang, X. et al. Viral and host factors related to the clinical outcome of COVID-19. Nature 583, 437-440, doi:10.1038/s41586-020-2355-0 (2020).

21 Issa, E., Merhi, G., Panossian, B., Salloum, T. \& Tokajian, S. SARS-CoV-2 and ORF3a: Nonsynonymous Mutations, Functional Domains, and Viral Pathogenesis. mSystems 5, doi:10.1128/mSystems.00266-20 (2020).

22 Young, B. E. et al. Effects of a major deletion in the SARS-CoV-2 genome on the severity of infection and the inflammatory response: an observational cohort study. Lancet (London, England) 396, 603-611, doi:10.1016/s0140-6736(20)31757-8 (2020).

23 Cao, Z. et al. Ubiquitination of SARS-CoV-2 ORF7a promotes antagonism of interferon response. Cellular \& molecular immunology 18, 746-748, doi:10.1038/s41423-020-00603$6(2021)$.

24 Nemudryi, A. et al. SARS-CoV-2 genomic surveillance identifies naturally occurring truncation of ORF7a that limits immune suppression. Cell reports 35, 109197, doi:10.1016/j.celrep.2021.109197 (2021).

25 Shen, L. et al. Emerging variants of concern in SARS-CoV-2 membrane protein: a highly conserved target with potential pathological and therapeutic implications. Emerging microbes \& infections 10, 885-893, doi:10.1080/22221751.2021.1922097 (2021).

26 Liu, J. et al. BNT162b2-elicited neutralization of B.1.617 and other SARS-CoV-2 variants. Nature 596, 273-275, doi:10.1038/s41586-021-03693-y (2021). 
27 Chen, R. E. et al. Resistance of SARS-CoV-2 variants to neutralization by monoclonal and serum-derived polyclonal antibodies. Nature medicine 27, 717-726, doi:10.1038/s41591021-01294-w (2021).

28 Zhang, L. et al. A proof of concept for neutralizing antibody-guided vaccine design against SARS-CoV-2. National Science Review 8, doi:10.1093/nsr/nwab053 (2021).

29 Chi, X. et al. A neutralizing human antibody binds to the N-terminal domain of the Spike protein of SARS-CoV-2. Science 369, 650-655, doi:10.1126/science.abc6952 (2020).

30 Shi, A. C. \& Xie, X. Making sense of spike D614G in SARS-CoV-2 transmission. Science China. Life sciences 64, 1062-1067, doi:10.1007/s11427-020-1893-9 (2021).

31 Wang, Z. et al. mRNA vaccine-elicited antibodies to SARS-CoV-2 and circulating variants. Nature 592, 616-622, doi:10.1038/s41586-021-03324-6 (2021).

$32 \mathrm{Li}, \mathrm{Q}$. et al. The Impact of Mutations in SARS-CoV-2 Spike on Viral Infectivity and Antigenicity. Cell 182, 1284-1294.e1289, doi:10.1016/j.cell.2020.07.012 (2020).

33 Liu, Z. et al. Identification of SARS-CoV-2 spike mutations that attenuate monoclonal and serum antibody neutralization. Cell host \& microbe 29, 477-488.e474, doi:10.1016/j.chom.2021.01.014 (2021).

34 Suryadevara, N. et al. Neutralizing and protective human monoclonal antibodies recognizing the N-terminal domain of the SARS-CoV-2 spike protein. Cell 184, 23162331.e2315, doi:10.1016/j.cell.2021.03.029 (2021).

35 Lopez Bernal, J. et al. Effectiveness of Covid-19 Vaccines against the B.1.617.2 (Delta) Variant. The New England journal of medicine 385, 585-594, doi:10.1056/NEJMoa2108891 (2021).

36 Cherian, S. et al. SARS-CoV-2 Spike Mutations, L452R, T478K, E484Q and P681R, in the Second Wave of COVID-19 in Maharashtra, India. Microorganisms 9, doi:10.3390/microorganisms9071542 (2021).

37 Zhang, Z. et al. SARS-CoV-2 spike protein dictates syncytium-mediated lymphocyte elimination. Cell death and differentiation 28, 2765-2777, doi:10.1038/s41418-021-007823 (2021).

38 GISAID.

Lineage

Details. https://covid19dashboard.regeneron.com/?tab=Lineage Details (2021).

39 Toldo, S. et al. Inflammasome formation in the lungs of patients with fatal COVID-19. Inflammation research : official journal of the European Histamine Research Society ... [et al.] 70, 7-10, doi:10.1007/s00011-020-01413-2 (2021).

40 Pereira, F. SARS-CoV-2 variants combining spike mutations and the absence of ORF8 may be more transmissible and require close monitoring. Biochemical and biophysical research communications 550, 8-14, doi:10.1016/j.bbrc.2021.02.080 (2021).

41 West, A. P., Jr. et al. Detection and characterization of the SARS-CoV-2 lineage B.1.526 in New York. Nature communications 12, 4886, doi:10.1038/s41467-021-25168-4 (2021).

42 Braun, K. M. et al. Transmission of SARS-CoV-2 in domestic cats imposes a narrow bottleneck. PLoS pathogens 17, e1009373, doi:10.1371/journal.ppat.1009373 (2021).

43 Hirotsu, Y. \& Omata, M. Detection of R.1 lineage severe acute respiratory syndrome coronavirus 2 (SARS-CoV-2) with spike protein W152L/E484K/G769V mutations in Japan. PLoS pathogens 17, e1009619, doi:10.1371/journal.ppat.1009619 (2021).

44 Molina-Mora, J. A. et al. SARS-CoV-2 genomic surveillance in Costa Rica: Evidence of a divergent population and an increased detection of a spike T1117I mutation. Infection, genetics and evolution : journal of molecular epidemiology and evolutionary genetics in infectious diseases 92, 104872, doi:10.1016/j.meegid.2021.104872 (2021). 
508

45 Gobeil, S. M. et al. Effect of natural mutations of SARS-CoV-2 on spike structure, conformation, and antigenicity. Science 373, doi:10.1126/science.abi6226 (2021).

46 Chen, J., Wang, R., Wang, M. \& Wei, G. W. Mutations Strengthened SARS-CoV-2 Infectivity. Journal of molecular biology 432, 5212-5226, doi:10.1016/j.jmb.2020.07.009 (2020).

47 Luo, R., Delaunay-Moisan, A., Timmis, K. \& Danchin, A. SARS-CoV-2 biology and variants: anticipation of viral evolution and what needs to be done. Environmental microbiology 23, 2339-2363, doi:10.1111/1462-2920.15487 (2021).

48 Yin, R. et al. Structural and energetic profiling of SARS-CoV-2 receptor binding domain antibody recognition and the impact of circulating variants. PLoS Comput Biol 17, e1009380, doi:10.1371/journal.pcbi.1009380 (2021).

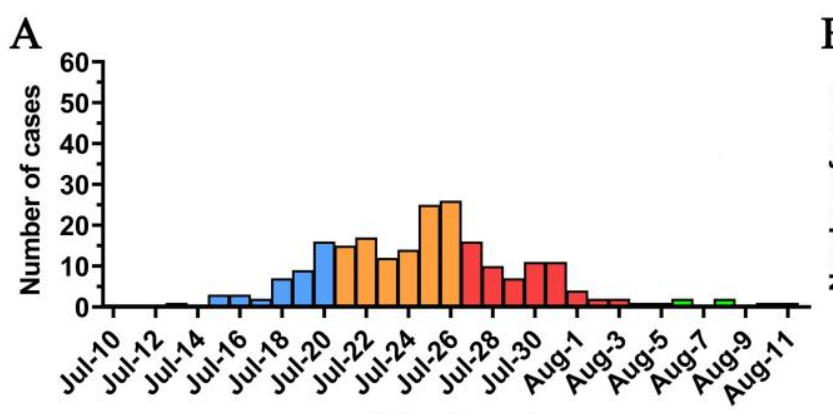

Date of onset

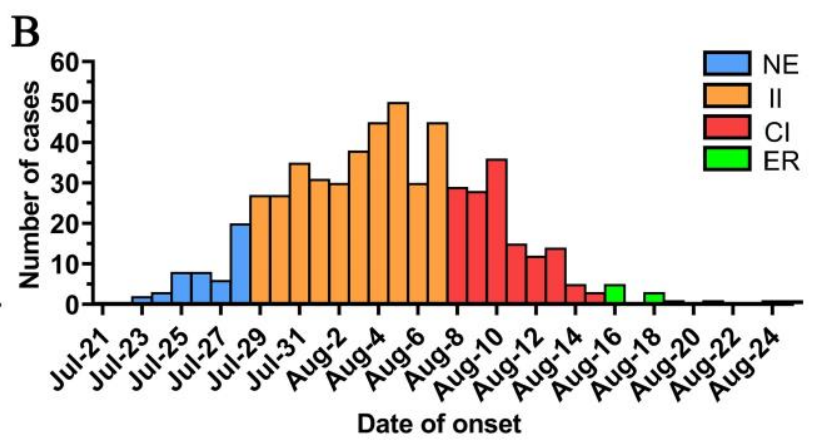

C
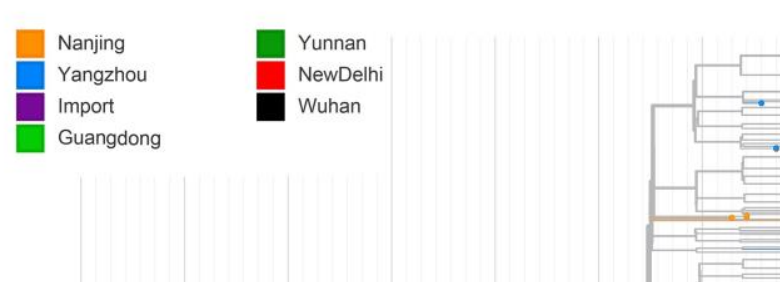

Date of onset

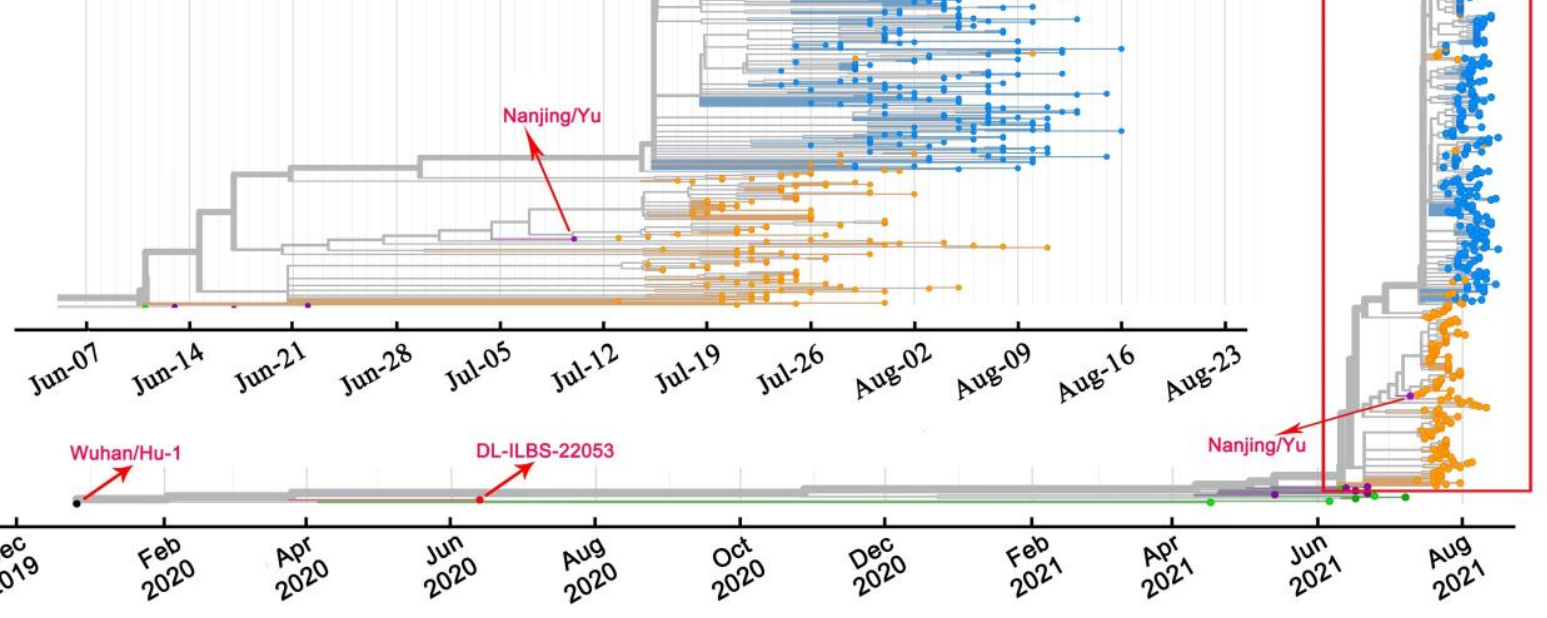

Fig.1. Distribution of COVID-19 cases and phylogenetic analysis of SARS-CoV-2 genome sequences in Nanjing (A) and Yangzhou (B), 2021. (A, B) Time series and laboratoryconfirmed COVID-19 cases in Nanjing and Yangzhou, respectively. Daily number of cases in natural epidemic period (NE), incompletely intervened period (II), completely intervened period (Cl), and epidemic regression period (ER) are shown in blue, yellow, red, and green, respectively. (C) The time resolved phylogenetic tree was constructed with the Nextstrain pipeline by including early imported COVID-19 cases detected before July 10, 2021 in Jiangsu province, delta variants of the local cases in Guangdong and Yunnan province detected in the period between March to July, 2021, and the early Delta variants DLILBS-22053 isolated in New Delhi, India. The sequences from the local COVID-19 cases of Nanjing (orange) and Yangzhou (blue) are highlighted with a red box and shown in panel $\mathrm{C}$ (inset). 
A

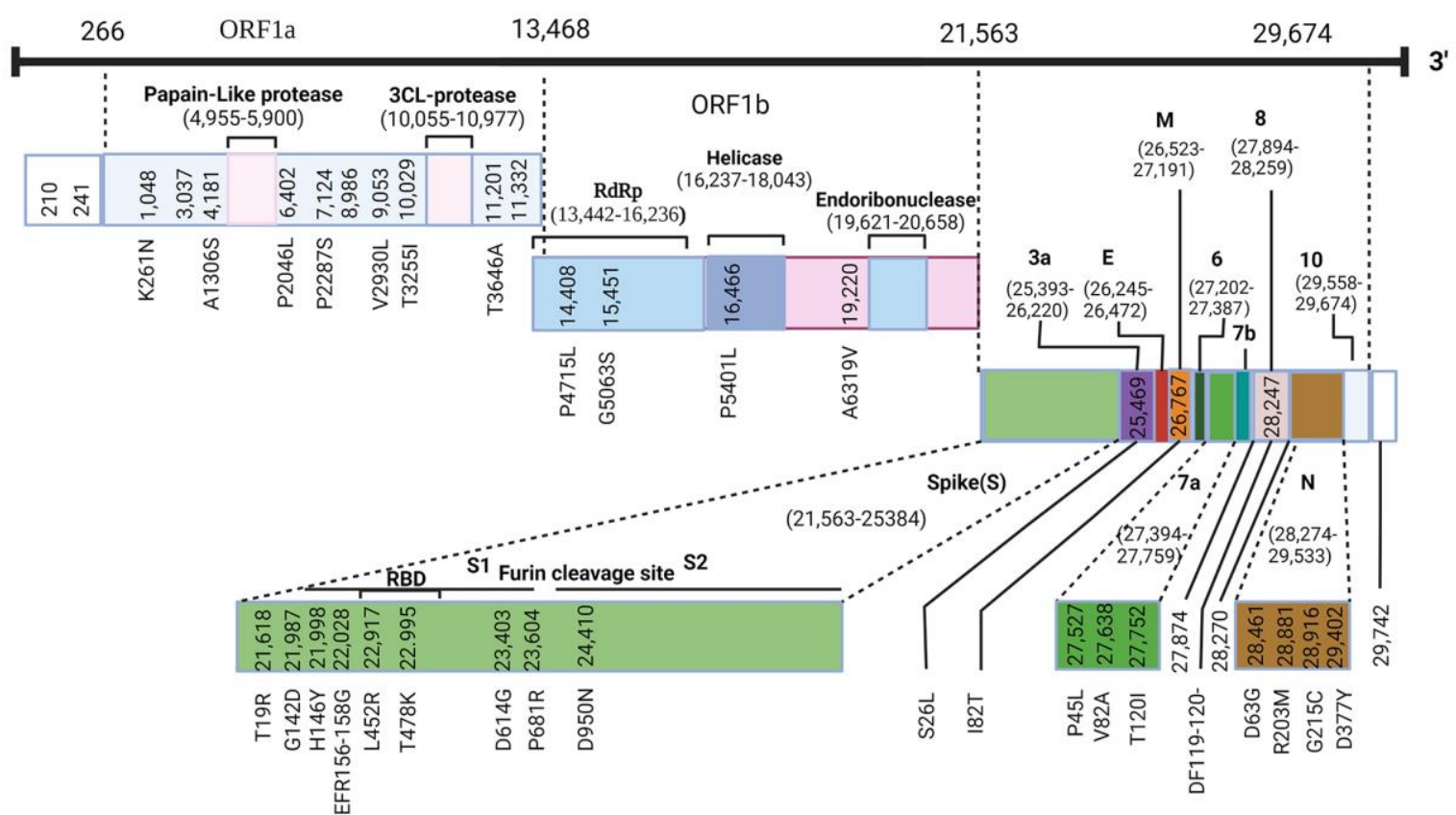

B

Receptor-binding Domain

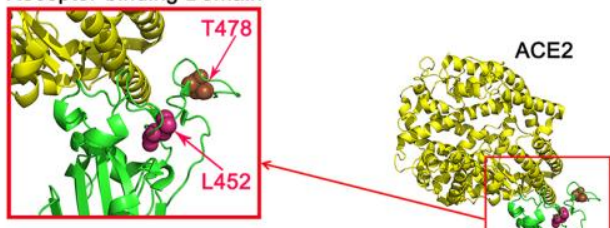

$\mathrm{N}$-terminal Domain

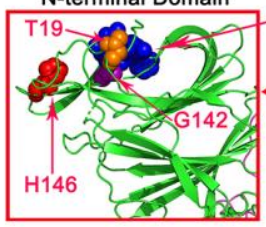

S2 Domain

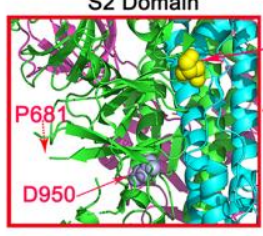

Fig. 2. Genomic variance of the case Nanjing/Yu and its impact on diagnosis. (A) The position of 38 variations in the genome. (B) Variations in the spike mapped to the spike trimer. Structure made by PyMOL using PDBID 7A94. (C) Mutations in the real time PCR detection primer region. G15451A mutation in RdRp resulted in the mismatch of Charite primer which may affect it's
C

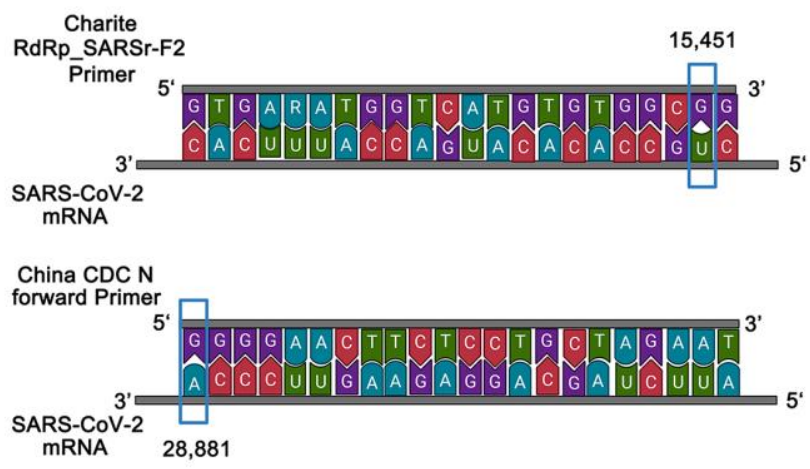

detection efficiency because it mapped to the $3^{\prime}$ primer region. Another G28881T mutation in $\mathrm{N}$ gene may have no effect on the efficiency of China CDC primer since it maps to the first nucleotide of the $5^{\prime}$ primer region. Fig. $2 \mathrm{~B}$ and Fig. $2 \mathrm{C}$ were made by Biorender (https://biorender.com/). 
A

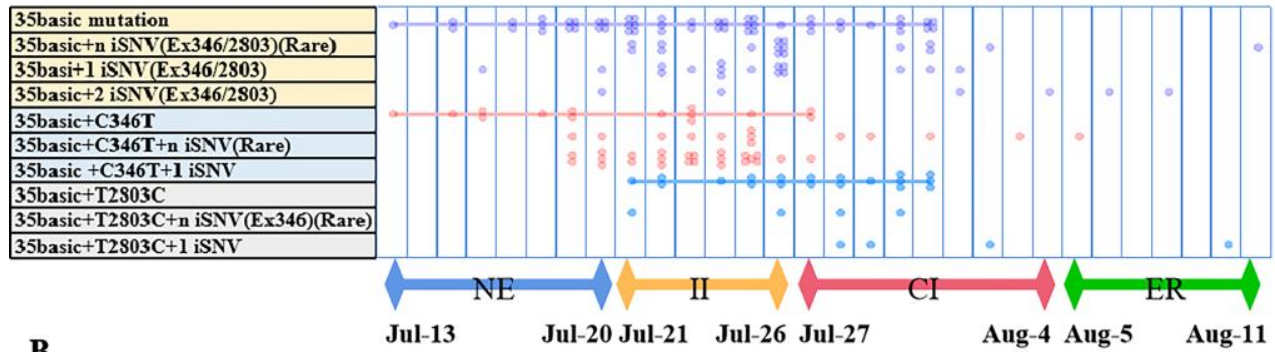

B

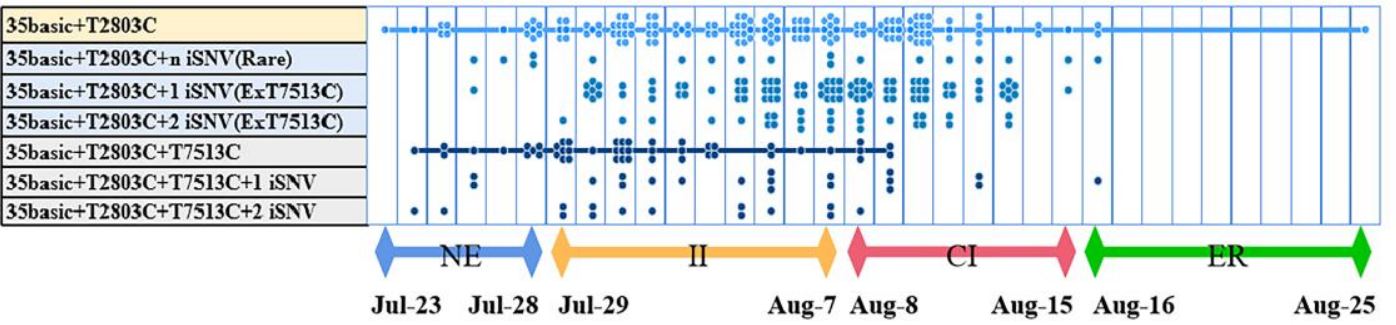

C

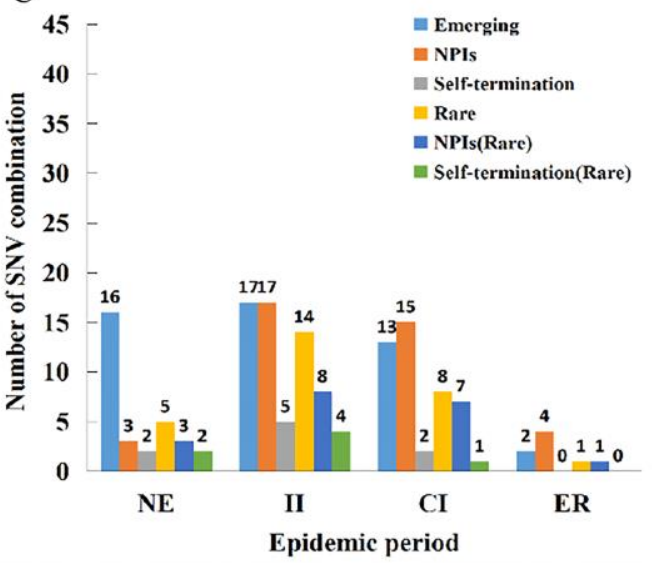

Fig. 3. SNV combinations under zero-Covid strategy. (A)

The distribution of different SNV combinations of Nanjing COVID-19 cases in different epidemic periods. The scatter indicated the different SNV combinations among the cases, and the connected line indicates that a certain SNV combination of mutations appears in related cases. (B) The distribution of different SNV combinations of Yangzhou COVID-19 cases in different epidemic periods. The scatter indicated the different SNV combinations among the cases, and the connected line indicates that a certain combination of mutations appears in related cases. (C) The number of SNV combinations formed in the 4 epidemic periods in Nanjing and that only appeared in a single case, the self-

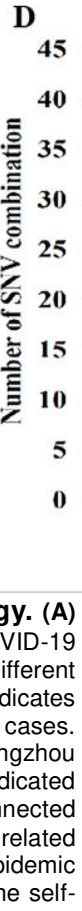


SIGuide list:

Materials and Methods

Statements of data and code availability

Acknowledgements

Details of author contributions

Competing interests

References

Extended data (Fig. 1, Tables 1 to 10)

Extended data Figure 1 | Temporal dynamics of intra-host populations in patients YZ404, YZ409 and YZ018.

Extended data Table 1 | Mutation sites of Delta variants from case Nanjing/Yu.

Extended data Table 2 | Distribution and observed effect of mutations and deletions in Spike protein.

Extended data Table 3 | Mutation Combinations of Nanjing.

Extended data Table 4 | Mutation Combinations of Yangzhou.

Extended data Table 5 | Summary table of Nanjing-Major combinations of mutations.

Extended data Table 6 | Summary table of Yangzhou-Major combinations of mutations.

Extended data Table 7 | SNV combinations can be terminated by NPI and vaccine.

Extended data Table 8 | Demographic characteristics of COVID-19 cases in Nanjing and Yangzhou.

Extended data Table 9| Key reagents.

Extended data Table 10 | Sample and sequencing information. Related to Figure 1C. 


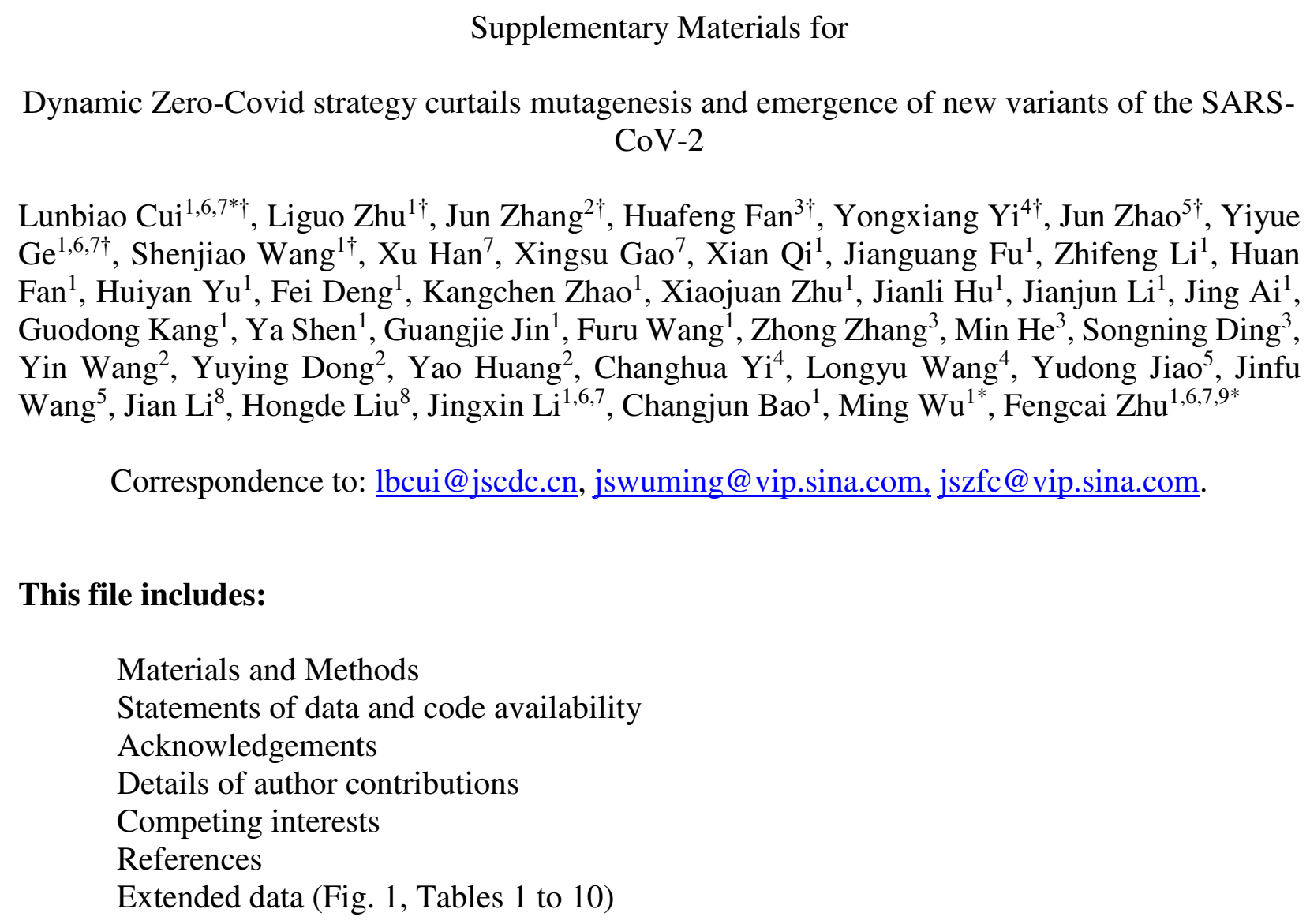

This file includes:

Materials and Methods

Statements of data and code availability

Acknowledgements

Details of author contributions

Competing interests

References 
Materials and Methods

$\underline{\text { Ethics }}$

$30 \quad$ Ethical approval for this study was approved by ethics committee of the Jiangsu Provincial Center for Disease Control and Prevention. Written informed consent was waived in light of the urgent need to collect data.

Epidemiological investigation of populations

COVID-19 cases in Nanjing and Yangzhou were included in this study, whose demographic characteristics were described in Extended data Table 8. Similar distribution of sex, CT values and clinical type were observed in Nanjing and Yangzhou, as well as age, except for the subgroup of '[40-50)' and ' $\geq 60$ '. Cases in Nanjing and Yangzhou were comparable regarding cluster distribution; 450/559 (80.50\%) cases were classified into clustered group in Yangzhou, while in Nanjing, this proportion was 32/221 (14.50\%). The differences are probably caused by differences in daily activities of the cases in Yangzhou. In addition, a significantly higher proportion of nonvaccination subgroup existed in Yangzhou when compared to Nanjing, may be for the reason that most of the cases in Yangzhou were the elderly or children who have not been immunized.

Verification of diagnosis and epidemiological investigation were conducted for each newly detected COVID-19 nucleic acid positive case. The verification of diagnosis included rechecking the first positive specimen and re-collecting the patient's nasal/pharyngeal swab specimens within 24 hours for nucleic acid testing. Patients with positive nucleic acid test at least twice were included in this study.

Epidemiological investigation includes the following four parts. (1) Investigation of clinical manifestations (whether there are symptoms, first symptoms and onset time) and the sampling time of the first positive nucleic acid test of COVID-19; (2) Activities of the infected person within 14 days before the onset time or the sampling time of first positive test, focus on investigating whether the contacts have been identified as infected, and investigating the date, duration, frequency, and protection of contact methods with the infected persons. In addition, exposure to objects or environment contaminated by infected persons, including exposure to foreign aircraft, exposure to environments in which known cases have been active or the contact with contaminated items (duration, frequency, exposure methods, protection conditions, and environmental characteristics). These activities help to determine the source of infection, assist in inferring the possible time of infection, and thus analyze the transmission relationship. (3) Close contacts, those who were in contact before symptom onset or 4 days before the first positive test, or those who were exposed to the same small confined space together with them, were defined as close contacts. (4) Sub-close contacts, a person who has been in contact with a close contact from contacting with an infected person to hotel quarantine was defined as a sub-close contact.

Through epidemiological investigation, clarify the transmission chain of the cluster epidemic and determine whether each infected person has continued transmission. If it is judged that there is no further spreader, it is determined as the last case of a transmission chain, and the infection period can be inferred based on the detection time or onset time of the last infected person. If a case has been quarantined before the infectious period, the termination of the variant transmission is considered to be due to non-drug intervention (NPI) measures. If a case has not been fully controlled during the infectious period but no transmission occurs, the termination of the variant transmission is considered to be due to self-termination of the virus.

$\underline{\text { Non-pharmaceutical interventions }}$ (NPI) 


\section{Nucleic Acid Extraction and Viral RNA Detection of SARS-CoV-2}

Upper respiratory specimens (nasopharyngeal and oropharyngeal swabs) were collected from all suspected cases. Total RNA was extracted from $200 \mu \mathrm{L}$ of the specimens using a magnetic infectious disease institutions within 2 hours for treatment, observation or quarantine, until they meet the discharge standards of the "Diagnosis and Treatment Protocol for COVID-19".

(2) Management of close contacts. Close contacts were transferred to the hotel quarantine within 12 hours after discovery, and take a single hotel room to quarantine until 14 days after contact with an infected person (people with difficulties in living on their own are allowed to be isolated at home or accompanied by someone), and respiratory samples were collected on the 1,4, 7, 14 days of the quarantine period for testing nucleic acid of SARS-CoV-2. Two nasopharyngeal swabs were collected on the 14th day, and were tested by two reagents separately. Those who tested negative during the quarantine period were released and be monitored at home for 7 days after release. They were not allowed to participate in gathering activities during the period and were suggested to take nucleic acid tests on the $2^{\text {nd }}$ day and $7^{\text {th }}$ day respectively, then the control would be lifted after all negative results of nucleic acid tests.

(3) Management of sub-close contacts. They were transferred to a centralized quarantine place within 12 hours and quarantine in a single room of a hotel for 7 days. If those close contacts they contact were tested negative within 4 days before quarantine, these sub-close contacts would be released after testing negative on the $1^{\text {st }}, 4^{\text {th }}$ or $7^{\text {th }}$ day. If those close contacts were tested positive, these sub-close contacts will be determined as close contacts for further control.

(4) Closed management of the community. Communities with community transmission or communities with more than 5 cases were closed for management. People in the communities were not allowed to leave their homes, and supplies were uniformly distributed after secondary protection by community management personnel. The nucleic acid tests were performed among persons in the communities on the $1^{\text {st }}, 4^{\text {th }}, 7^{\text {th }}$ and $14^{\text {th }}$ day of management. The control would be lifted until no new cases appear in the community over 14 days after the cases left the community.

(5) Containment management of the community. The communities where cases have occurred, then people in the community are not allowed to leave the community, while one person in each family is allowed to purchase daily necessities in or near the community. The control would be lifted until no new case appear in the community over 14 days after the cases left the community and all persons in the community are negative by nucleic acid screening within 14 days.

(6) Other social management. Districts and counties affected by the epidemic were temporarily closed all business places, such as chess and card rooms, activity rooms, cinemas, KTV, bars, amusement parks, museums and other leisure places that are not necessary for daily life, while other places that are necessary for daily life will take control measures such as limiting traffic, shortening and adjusting business hours.

(7) Nucleic acid screening: nucleic acid screening was carried out for all members of communities involved in the occurrence and activities of cases, so as to determine the spread of infection and further adjust the scope of control. During the epidemic period, all members were screened for nucleic acid every 3 to 5 days. Whether to carry out the next round of testing and the time and scope of testing was determined according to the test results and the development trend of the epidemic. If there were newly infected persons who were not under control, screening would continue to be carried out in their activity areas. If all new cases occur in the controlled population, screening would not be carried out. 
bead-based viral RNA nucleic acid extraction system (TianLong Technologies, Xian, China). Then, qRT-PCR was performed using a 2019-nCoV RNA detection kit (BioGerm Medical Technologies, Shanghai, China). Conditions for the reaction were as follows: $50^{\circ} \mathrm{C}$ for $10 \mathrm{~min}$ and $95^{\circ} \mathrm{C}$ for 5 min, followed by 45 cycles of amplification at $95^{\circ} \mathrm{C}$ for $10 \mathrm{~s}$ and $55^{\circ} \mathrm{C}$ for $40 \mathrm{~s}$. Amplification and detection were performed with the QuantStudio7 Pro Real-Time PCR Systems (Thermo Fisher Scientific, MA, USA) according to the manufacturer's instructions. Data were analyzed using the software supplied by the manufacturer. Sample was positive if the cycling threshold (CT) values of qRT-PCR for the ORF1ab and the N genes were less than 37. Sample was deemed negative if no CT value, or CT value of greater than 40, or unrepeatable CT value in the range of $37-40$ were obtained. Tests meeting both ORF1ab and NP gene positivity criterion simultaneously were considered positive.

\section{Virus amplification and sequencing}

For samples with qRT-PCR Ct value $\leq 34$, whole genome was amplified using target specific multiplex PCR amplification kit (Beijing MicroFuture, BAIYITECH, MGI, China, and Thermo Fisher, USA) according to manufacturer's instructions. PCR products were purified using AmpureXP beads (Beckman Coulter, USA) and quantified using fluorimetry with the Qubit dsDNA High Sensitivity assay on the Qubit 2.0 instrument (Life Technologies, USA) (Extended data Table 9). After DNA sequencing Library was constructed, sequencing of libraries was conducted on the Illumina (Miseq, MiniSeq, NextSeq2000, and iSeq100), BGI (MGISEQ-2000), Thermo Fisher (S5XL and Genexus ${ }^{\mathrm{TM}}$ ) sequencing platform (Extended data Table 10). The original sequencing data were firstly cut off the amplification primer sequences. Then, reads were trimmed to remove low-quality bases and assembled into a genome consensus sequences using Wuhan-Hu-1 (GenBank:MN908947.3) as reference in the CLC Genomics Workbench (Version 21.0) software. For variant detection, base quality larger than 20, the minimum coverage 100, count 10 and frequency $10 \%$ were set. Variants were called also using Wuhan-Hu-1 as reference.

\section{Phylogenetic analysis}

A total of 537 (167 cases from Nanjing outbreak, 353 cases from Yangzhou outbreak, seven imported cases related to Delta variants, four cases from Guangdong outbreak and four cases from the Yunnan outbreak of Delta variants, Wuhan-Hu-1, and the early Delta variants DL-ILBS-22053 listed in Extended data Table 10) near-complete and complete genomes were aligned using MAFFT v7.487. ${ }^{1}$ The resulting dataset were used to estimate maximum likelihood (ML) phylogeny using IQTree V. $2^{2}$. The time resolved phylogenetic tree was constructed with the Nextstrain pipeline ${ }^{3}$.

$\underline{\text { Statistical analyses }}$

In this real-world study, we included all 520 cases from which genome-wide data were obtained during an outbreak in Nanjing and Yangzhou from July 13 to August 25, 2021. Demographic and laboratory data were obtained from the stream database, and the combined mutation data for each case were summarized and compared. Descriptive analyses of demographic data are presented as mean when continuous and as frequency and ratio (\%) when categorical. Univariate and multivariate logistic regression methods were used to investigate the factors influencing whether the SNV combinations could be terminated. Six variables were selected for 164 multivariate analysis based on preliminary statistical findings and clinical limitations. Statistical 
analyses were done using IBM SPSS18.0 and R (Version 9.3). A two-sided $\alpha$ of less than 0.05 was considered statistically significant.

167 Statements of data and code availability: All sequencing reads after primer trimming have been submitted to the National Genomics Data Center (https://ngdc.cncb.ac.cn/) with submission number CRA005588. The generated consensus sequences were submitted with accession number GWHBGCG01000000-GWHBGWF01000000. All other data are available in the manuscript or the supplementary materials, and the results supporting the findings in this study are available upon request from the corresponding authors.

Acknowledgements: We thank Jingyu Lou, Yue Ding and Jinmei Qian for their support with the sequencing and quality control, Nan Zhou, Xin Zhou, Jie Ding, Qin Xu, Tao Ma, Xiang Huo, Yue Dai, Yang Zhou, Lingen Shi, Na Sun, Wei Wang, Chuanwu Sun, Yalong Wang, Yanmin Zhou, Liling Chen, Zhiquan Wang, Yanmin Mao, Zheng Zhang, Hao Xue, Cuiying Chen and Lei Wang for technical assistance with epidemiological investigation. We thank the Jiangsu Provincial Key Research and Development Program BE2021738 and National science foundation of Jiangsu province (BK2021373, BE2019761).

Details of author contributions: FC.Z., and LB.C., conceptualized and provided funding for this study, LB.C., LG.Z., YY.G., SJ. W., X.H., XS.G., X.Q., JG.F., ZF.L., H.F., HY. Y., F.D., KC.Z., XJ.Z., J.L., and HD.L., did the methodology part, J.Z., HF.F., YX.Y., J.Z., JL.H., JJ.L., J.A., GD.K., Y.S., GJ.J., FR.W., Z.Z., M.H., SN.D., Y.W., YU.D., HM.Y., CH.Y., LY.W., YD.J., JF.W., JX.L., CJ.B., and M.W., investigated the data, M.W., FC.Z., and LB.C administrated this project, LB.C., X.H., and XS.G. wrote the original draft, FC.Z., and LB.C review and edit this paper. LB.C., LG.Z., J.Z., HF.F., YX.Y., J.Z., YY.G., and SJ.W. contributed equally to this study.

Competing interests: All authors declare no competing interests.

\section{References}

1 Katoh, K. \& Standley, D. M. MAFFT multiple sequence alignment software version 7: improvements in performance and usability. Molecular biology and evolution 30, 772-780, doi:10.1093/molbev/mst010 (2013).

2 Trifinopoulos, J., Nguyen, L. T., von Haeseler, A. \& Minh, B. Q. W-IQ-TREE: a fast online phylogenetic tool for maximum likelihood analysis. Nucleic acids research $\mathbf{4 4}$, W232-235, doi:10.1093/nar/gkw256 (2016).

3 Hadfield, J. et al. Nextstrain: real-time tracking of pathogen evolution. Bioinformatics (Oxford, England) 34, 4121-4123, doi:10.1093/bioinformatics/bty407 (2018).

4 Liu, J. et al. BNT162b2-elicited neutralization of B.1.617 and other SARS-CoV-2 variants. Nature 596, 273-275, doi:10.1038/s41586-021-03693-y (2021).

5 Chen, R. E. et al. Resistance of SARS-CoV-2 variants to neutralization by monoclonal and serum-derived polyclonal antibodies. Nature medicine 27, 717-726, doi:10.1038/s41591021-01294-w (2021).

6 Zhang, L. et al. A proof of concept for neutralizing antibody-guided vaccine design against SARS-CoV-2. National Science Review 8, doi:10.1093/nsr/nwab053 (2021).

209 Chi, X. et al. A neutralizing human antibody binds to the N-terminal domain of the Spike protein of SARS-CoV-2. Science 369, 650-655, doi:10.1126/science.abc6952 (2020).

8 Suryadevara, N. et al. Neutralizing and protective human monoclonal antibodies 
recognizing the N-terminal domain of the SARS-CoV-2 spike protein. Cell 184, 23162331.e2315, doi:10.1016/j.cell.2021.03.029 (2021).
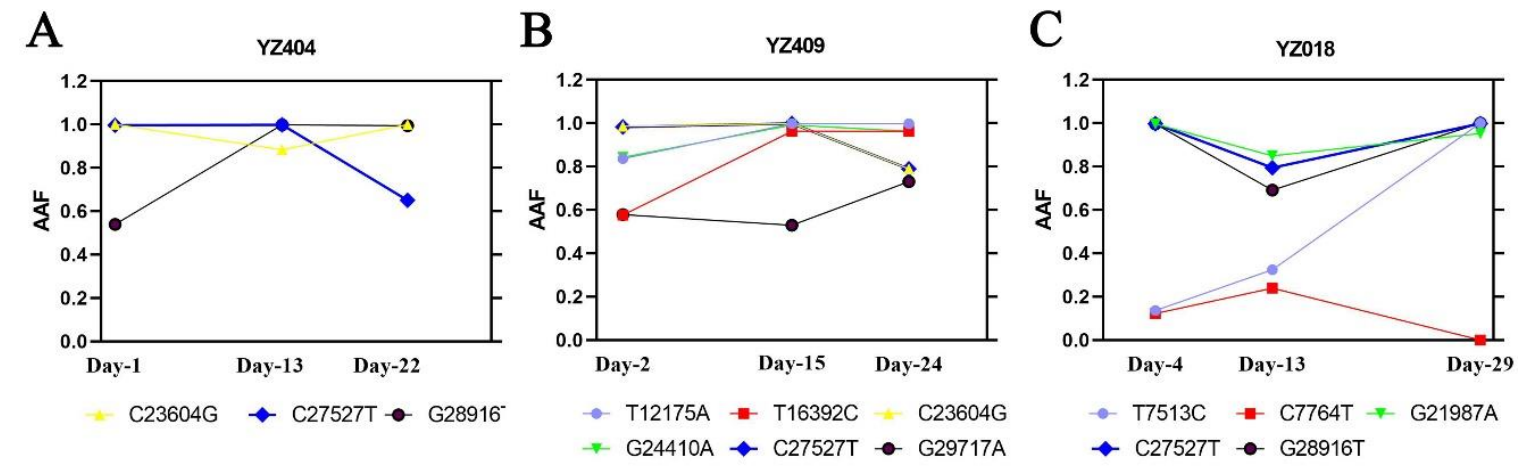

9 Greaney, A. J. et al. Complete Mapping of Mutations to the SARS-CoV-2 Spike ReceptorBinding Domain that Escape Antibody Recognition. Cell host \& microbe 29, 44-57.e49, doi:10.1016/j.chom.2020.11.007 (2021).

10 Liu, Z. et al. Identification of SARS-CoV-2 spike mutations that attenuate monoclonal and serum antibody neutralization. Cell host \& microbe 29, 477-488.e474, doi:10.1016/j.chom.2021.01.014 (2021).

11 Wang, Z. et al. mRNA vaccine-elicited antibodies to SARS-CoV-2 and circulating variants. Nature 592, 616-622, doi:10.1038/s41586-021-03324-6 (2021).

$12 \mathrm{Li}, \mathrm{Q}$. et al. The Impact of Mutations in SARS-CoV-2 Spike on Viral Infectivity and Antigenicity. Cell 182, 1284-1294.e1289, doi:10.1016/j.cell.2020.07.012 (2020).

13 Shi, A. C. \& Xie, X. Making sense of spike D614G in SARS-CoV-2 transmission. Science China. Life sciences 64, 1062-1067, doi:10.1007/s11427-020-1893-9 (2021).

14 Lopez Bernal, J. et al. Effectiveness of Covid-19 Vaccines against the B.1.617.2 (Delta) Variant. The New England journal of medicine 385, 585-594, doi:10.1056/NEJMoa2108891 (2021).

\section{Extended data Figure 1| Temporal dynamics of intra-host populations in patients YZ404, YZ409 and YZ018. Alternative allele} frequencies (AAFs) among sampling dates in patients YZ404 (A), YZ409 (B) and YZ018 (C). Days post the first symptom date are shown in the abscissa. Colors represent different iSNVs. 


\section{Extended data Table 1| Mutation sites of Delta variants from case Nanjing/Yu.}

\begin{tabular}{|c|c|c|}
\hline Mutation site & Protein (number of substitutions) & INFO \\
\hline 2019-nCoV_210 & ORF1ab (16) & VEP=upstream gene variant, DISTANCE=56, QHD43415.1,gene-orf1ab \\
\hline 2019-nCoV_241 & & VEP=upstream gene variant, DISTANCE=25, QHD43415.1,gene-orf1ab \\
\hline 2019-nCoV_3037 & & VEP=synonymous_variant,QHD43415.1:p.924F,gene-orf1ab:c.2772ttC>ttT \\
\hline 2019-nCoV_8986 & & VEP=synonymous_variant,QHD43415.1:p.2907D,gene-orf1ab:c.8721gaC>gaT \\
\hline 2019-nCoV_11332 & & VEP=synonymous_variant,QHD43415.1:p.3689V,gene-orf1ab:c.11067gtA>gtG \\
\hline 2019-nCoV_1048 & & VEP=missense_variant,QHD43415.1:p.261K>N,gene-orf1ab:c.783aaG $>$ aaT \\
\hline 2019-nCoV_4181 & & VEP=missense_variant,QHD43415.1:p.1306A>S, gene-orf1ab:c.3916Gct>Tct \\
\hline 2019-nCoV_6402 & & VEP=missense_variant, QHD43415.1:p.2046P>L,gene-orf1ab:c.6137cCa $>$ CTa \\
\hline 2019-nCoV_7124 & & VEP=missense_variant,QHD43415.1:p.2287P >S, gene-orf1ab:c.6859Cct>Tct \\
\hline 2019-nCoV_9053 & & VEP=missense_variant,QHD43415.1:p.2930V >L,gene-orf1ab:c.8788Gta>Tta \\
\hline 2019-nCoV_10029 & & VEP=missense_variant,QHD43415.1:p.3255T>I,gene-orf1ab:c.9764aCc>aTc \\
\hline 2019-nCoV_11201 & & VEP=missense_variant,QHD43415.1:p.3646T>A,gene-orf1ab:c.10936Act>Gct \\
\hline 2019-nCoV_14408 & & VEP=missense_variant,QHD43415.1:p.4715P>L,gene-orf1ab:c.14144cCt>cTt \\
\hline 2019-nCoV_15451 & & VEP=missense_variant,QHD43415.1:p.5063G>S,gene-orf1ab:c.15187Ggt>Agt \\
\hline 2019-nCoV_16466 & & VEP=missense_variant,QHD43415.1:p.5401P>L,gene-orf1ab:c.16202cCa>cTa \\
\hline 2019-nCoV_19220 & & VEP=missense_variant,QHD43415.1:p.6319A $>\mathrm{V}$, gene-orf1ab:c.18956gCt>gTt \\
\hline 2019-nCoV_21618 & Spike (8) & VEP=missense_variant,QHD43416.1:p.19T>R,gene-S:c.56aCa>aGa \\
\hline 2019-nCoV_21987 & & VEP=missense_variant,QHD43416.1:p.142G>D,gene-S:c.425gGt>gAt \\
\hline 2019-nCoV_21998 & & VEP=missense_variant,QHD43416.1:p.146H>Y,gene-S:c.436Cac>Tac \\
\hline 2019-nCoV_22917 & & VEP=missense_variant,QHD43416.1:p.452L>R,gene-S:c.1355cTg>cGg \\
\hline 2019-nCoV_22995 & & VEP=missense_variant,QHD43416.1:p.478T>K,gene-S:c.1433aCa>aAa \\
\hline 2019-nCoV_23403 & & VEP=missense_variant,QHD43416.1:p.614D>G,gene-S:c.1841gAt>gGt \\
\hline 2019-nCoV_23604 & & VEP=missense_variant,QHD43416.1:p.681P>R,gene-S:c.2042cCt>cGt \\
\hline
\end{tabular}


2019-nCoV_24410

2019-nCoV_25469

2019-nCoV 26767

2019-nCoV_27527

2019-nCoV 27638

2019-nCoV_27752

2019-nCoV_27874

2019-nCoV_28461

2019-nCoV_28881

2019-nCoV_28916

2019-nCoV 29402

2019-nCoV_29742
VEP=missense_variant,QHD43416.1:p.950D>N,gene-S:c.2848Gat>Aat

ORF3a (1)

Membrane (1)

ORF7a (3)

ORF8 (1)

Nucleocapsid

(4)

VEP=missense_variant,QHD43417.1:p.26S>L,gene-ORF3a:c.77tCa>tTa

VEP=missense_variant,QHD43419.1:p.82I>T,gene-M:c.245aTc>aCc

VEP=missense_variant,QHD43421.1:p.45P>L,gene-ORF7a:c.134cCa>cTa

VEP=missense_variant,QHD43421.1:p.82V>A,gene-ORF7a:c.245gTt>gCt

VEP=missense_variant,QHD43421.1:p.120T>I,gene-ORF7a:c.359aCa>aTa

VEP=upstream gene variant, DISTANCE=20,QHD43422.1,gene-ORF8

VEP=missense_variant,QHD43423.2:p.63D>G,gene-N:c.188gAc>gGc

VEP=missense_variant,QHD43423.2:p.203R>M,gene-N:c.608aGg>aTg

VEP=missense_variant,QHD43423.2:p.215G>C,gene-N:c.643Ggt>Tgt

VEP=missense_variant,QHD43423.2:p.377D>Y,gene-N:c.1129Gat>Tat

ORF10 (1) 
Extended data Table 2 | Distribution and observed effect of mutations and deletions in Spike protein.

\begin{tabular}{|c|c|c|}
\hline Mutation site & Effects & Ref. \\
\hline T19R & - & - \\
\hline G142D & G142D reducing neutralization by the BNT162b2 immune sera ${ }^{4}$ and the $m A b 2489^{5}$. & $\begin{array}{l}\text { [4] Nature. } 2021 ; 10.1038 / s 41586-021-03693-y . \\
\text { [5] Nat Med. } 2021 ; 27(4): 717-726 .\end{array}$ \\
\hline $\mathrm{H} 146 \mathrm{Y}$ & $\mathrm{H} 146 \mathrm{Y}$ reducing neutralization by the $\mathrm{mAb} \mathrm{FC} 05^{6}$ and $4 \mathrm{~A} 8^{7}$. & $\begin{array}{l}\text { [6] National Science Review. 2021; nwab053. [7] Science. 2020; 369(6504):650- } \\
655 .\end{array}$ \\
\hline$\triangle \mathrm{E} 156$ & - & - \\
\hline$\triangle \mathrm{F} 157$ & F157A reducing neutralization by the $\mathrm{mAb} 2489^{8}$. & [8] Cell. 2021;184(9):2316-2331. \\
\hline R158G & - & - \\
\hline L452R & $\begin{array}{l}\text { L452R reducing neutralization by the mAb FC08 }{ }^{6}, \mathrm{COV}_{2}-2096^{9}, \mathrm{SARS} 2-01, \mathrm{SARS} 2-02 \text { and SARS2- } \\
32^{10}, \mathrm{C} 643 \text { and } \mathrm{C}^{2} 28^{11} \text {, and convalescent plasma }{ }^{12} \text {. }\end{array}$ & $\begin{array}{l}\text { [8] National Science Review. 2021; nwab053. } \\
\text { [9] Cell Host Microbe. 2021;29(1):44-57. } \\
\text { [10] Cell Host Microbe. 2021;29(3):477-488. } \\
\text { [11] Nature. 2021;592(7855):616-622. } \\
\text { [12] Cell. 2020;182(5):1284-1294. }\end{array}$ \\
\hline T478K & $\begin{array}{l}\text { T478I reducing neutralization by the mAb SARS2-16, SARS2-19 and and convalescent plasma ( } 13 \text { and } \\
35 \#)^{.0}\end{array}$ & [10] Cell Host Microbe. 2021;29(3):477-488. \\
\hline $\mathrm{D} 614 \mathrm{G}$ & $\begin{array}{l}\text { D614G could enhance virus infectivity and thermal stability, D614G does not significantly affect the } \\
\text { effectiveness of existing vaccines, but may weaken the neutralizing activity of certain specific } \\
\text { antibodies. }{ }^{13}\end{array}$ & [13] Sci China Life Sci. 2021;64(7):1062-1067. \\
\hline P681R & P681R may have increased replication, which leads to higher viral loads and increased transmission. ${ }^{14}$ & [14] N Engl J Med. 2021; 385(7): 585-594. \\
\hline D950N & - & - \\
\hline
\end{tabular}


Extended data Table 3 | Mutation Combinations of Nanjing.

\begin{tabular}{|c|c|c|c|c|c|}
\hline Combination of Mutation & $\begin{array}{l}\text { Date of onset of } \\
\text { first case }\end{array}$ & $\begin{array}{l}\text { Date of onset of } \\
\text { last case }\end{array}$ & $\begin{array}{l}\text { Total number of cases } \\
\text { (controlled/un- } \\
\text { controlled) }\end{array}$ & $\begin{array}{l}\text { Number of } \\
\text { cases in } \\
\text { control period }\end{array}$ & $\begin{array}{l}\text { Number of cases in } \\
\text { uncontrolled period }\end{array}$ \\
\hline Basic Mutation (same with Nanjing/Yu) & $2021 / 7 / 13$ & $2021 / 7 / 31$ & $51(28 / 23)$ & 26 & 25 \\
\hline С8819T & $2021 / 7 / 26$ & $2021 / 7 / 26$ & $1(0 / 1)$ & 0 & 1 \\
\hline T2803C & $2021 / 7 / 29$ & $2021 / 7 / 31$ & $19(14 / 5)$ & 11 & 8 \\
\hline T2803CC15738T & $2021 / 7 / 28$ & $2021 / 7 / 28$ & $1(1 / 0)$ & 1 & 0 \\
\hline T2803CG16968T & $2021 / 7 / 28$ & $2021 / 8 / 2$ & $2(1 / 1)$ & 2 & 0 \\
\hline A27133T & $2021 / 7 / 31$ & $2021 / 7 / 31$ & $1(1 / 0)$ & 1 & 0 \\
\hline C1060TC14573TC14576GT14562C & $2021 / 7 / 26$ & $2021 / 7 / 26$ & $1(1 / 0)$ & 0 & 1 \\
\hline C1385T & $2021 / 7 / 22$ & $2021 / 7 / 22$ & $1(1 / 0)$ & 0 & 1 \\
\hline C16289T & $2021 / 7 / 31$ & $2021 / 7 / 31$ & $1(1 / 0)$ & 1 & 0 \\
\hline C16289TA2467G & $2021 / 8 / 1$ & $2021 / 8 / 4$ & $2(2 / 0)$ & 2 & 0 \\
\hline C17402T & $2021 / 8 / 2$ & $2021 / 8 / 2$ & $1(1 / 0)$ & 1 & 0 \\
\hline C18828T & $2021 / 7 / 20$ & $2021 / 7 / 26$ & $6(5 / 1)$ & 0 & 6 \\
\hline C22642T & $2021 / 7 / 22$ & $2021 / 7 / 24$ & $2(1 / 1)$ & 0 & 2 \\
\hline C22642TC9165T & $2021 / 7 / 30$ & $2021 / 7 / 30$ & $1(1 / 0)$ & 1 & 0 \\
\hline C346T & $2021 / 7 / 13$ & $2021 / 7 / 27$ & $14(8 / 6)$ & 2 & 12 \\
\hline C346TA6411G & $2021 / 7 / 20$ & $2021 / 7 / 23$ & $2(2 / 0)$ & 0 & 2 \\
\hline С346TC1060T & $2021 / 7 / 20$ & $2021 / 7 / 25$ & $9(7 / 2)$ & 0 & 9 \\
\hline С346TC1060TC14708T & $2021 / 7 / 25$ & $2021 / 7 / 25$ & $1(1 / 0)$ & 0 & 1 \\
\hline С346TC1060TT2803C & $2021 / 7 / 25$ & $2021 / 7 / 25$ & $1(1 / 0)$ & 0 & 1 \\
\hline С346TC18828TC1060T & $2021 / 7 / 25$ & $2021 / 7 / 25$ & $1(1 / 0)$ & 0 & 1 \\
\hline С346ТС2623Т & $2021 / 7 / 23$ & $2021 / 7 / 23$ & $1(0 / 1)$ & 0 & 1 \\
\hline С346TC28687T & $2021 / 7 / 20$ & $2021 / 7 / 20$ & $1(1 / 0)$ & 0 & 1 \\
\hline С346ТС8772Т & $2021 / 7 / 19$ & $2021 / 7 / 27$ & $6(3 / 3)$ & 1 & 5 \\
\hline C346TG11083T & $2021 / 7 / 20$ & $2021 / 7 / 26$ & $5(3 / 2)$ & 0 & 5 \\
\hline C346TG28209T & $2021 / 7 / 19$ & $2021 / 7 / 25$ & $2(1 / 1)$ & 0 & 2 \\
\hline C346TG28209TA25934C & $2021 / 7 / 22$ & $2021 / 7 / 29$ & $3(2 / 1)$ & 1 & 2 \\
\hline C346TG28209TA25934CC17822T & $2021 / 8 / 3$ & $2021 / 8 / 5$ & $2(2 / 0)$ & 2 & 0 \\
\hline C346TG28209TA25934CT7513C & $2021 / 7 / 31$ & $2021 / 7 / 31$ & $1(1 / 0)$ & 1 & 0 \\
\hline C346TC8772TG5629T & $2021 / 7 / 28$ & $2021 / 7 / 28$ & $1(1 / 0)$ & 1 & 0 \\
\hline
\end{tabular}


T26259C

2021/7/21

2021/7/21

T26259CC 1385T

2021/7/20

$2021 / 7 / 24$

T26259CG472A

2021/8/6

2021/8/8

T26259CG472AC16192A

2021/8/11

2021/8/11

T26259CG246T

\section{A13566G}

C22642TT2803CA15201GC14573TC14576GG26951CT14562C

$2021 / 7 / 21$

$2021 / 7 / 21$

$2021 / 7 / 19$

$2021 / 7 / 19$

\section{C346TC22642T}

2021/7/30

T2803CG23868T

$2021 / 7 / 26$

2021/7/30

$2021 / 7 / 29$

\section{$2021 / 7 / 26$}

Combination of Mutation: The emergence of Combination of Mutation in Nanjing.

Date of occurrence of first case: Date of the earliest case among the cases with the Combination of Mutation.

Date of occurrence of last case: Date of the last case among the cases with the Combination of Mutation.

Total number of cases (controlled/uncontrolled): total number of cases of this mutation combination in Nanjing.

Controlled number: onset date of cases after NPI measures (include same date). uncontrolled number: onset date of cases before NPI measures.

249 Number of cases in control period: Number of cases with onset dates in $\mathrm{Cl}$ and $\mathrm{ER}$ stage.

250 Number of cases in uncontrolled period: Number of cases with onset dates in NE and II. 
Extended data Table 4 | Mutation Combinations of Yangzhou.

\begin{tabular}{|c|c|c|c|c|c|}
\hline Combination of Mutation & $\begin{array}{l}\text { Date of onset of } \\
\text { first case }\end{array}$ & $\begin{array}{l}\text { Date of onset of } \\
\text { last case }\end{array}$ & $\begin{array}{l}\text { Total number of } \\
\text { cases (controlled } \\
\text { /uncontrolled) }\end{array}$ & $\begin{array}{l}\text { Number of } \\
\text { cases in } \\
\text { control } \\
\text { period }\end{array}$ & $\begin{array}{l}\text { Number of } \\
\text { cases in } \\
\text { uncontrolled } \\
\text { period }\end{array}$ \\
\hline Т2803СС2939Т & $2021 / 7 / 26$ & $2021 / 8 / 1$ & $6(4 / 2)$ & 6 & 0 \\
\hline C22642TT2803CG5629T & $2021 / 8 / 9$ & $2021 / 8 / 9$ & $1(0 / 1)$ & 0 & 1 \\
\hline G11083TT2803C & $2021 / 8 / 10$ & 2021/8/10 & $1(0 / 1)$ & 0 & 1 \\
\hline G11083TT2803CC23525T & $2021 / 8 / 10$ & 2021/8/11 & $2(1 / 1)$ & 0 & 2 \\
\hline G11083TT2803CT7513CC18486TG10533T & $2021 / 7 / 30$ & $2021 / 7 / 30$ & $1(0 / 1)$ & 1 & 0 \\
\hline Т2803СC5497T & $2021 / 8 / 6$ & $2021 / 8 / 6$ & $1(1 / 0)$ & 1 & 0 \\
\hline T2803CG3880A & $2021 / 8 / 9$ & $2021 / 8 / 10$ & $3(1 / 2)$ & 0 & 3 \\
\hline T2803C & $2021 / 7 / 23$ & $2021 / 8 / 25$ & $140(75 / 65)$ & 95 & 45 \\
\hline T2803CA15201GG26951C & $2021 / 8 / 5$ & $2021 / 8 / 13$ & $4(3 / 1)$ & 2 & 2 \\
\hline T2803CA15201GG26951CG26754T & $2021 / 8 / 10$ & $2021 / 8 / 10$ & $1(0 / 1)$ & 0 & 1 \\
\hline T2803CA15201GG26951CG26754TG28514T & $2021 / 8 / 16$ & $2021 / 8 / 16$ & $1(0 / 1)$ & 0 & 1 \\
\hline T2803CA3908GC19955TA156G & $2021 / 8 / 11$ & $2021 / 8 / 11$ & $1(1 / 0)$ & 0 & 1 \\
\hline T2803CA5289T & $2021 / 8 / 8$ & $2021 / 8 / 8$ & $1(1 / 0)$ & 0 & 1 \\
\hline T2803CA3908G & $2021 / 7 / 31$ & $2021 / 8 / 13$ & $11(8 / 3)$ & 5 & 6 \\
\hline T2803CA3908GG4399T & $2021 / 8 / 4$ & $2021 / 8 / 7$ & $2(1 / 1)$ & 2 & 0 \\
\hline T2803CA3908GG5953T & $2021 / 8 / 7$ & $2021 / 8 / 7$ & $1(1 / 0)$ & 1 & 0 \\
\hline T2803CA7300T & $2021 / 7 / 30$ & $2021 / 8 / 6$ & $4(3 / 1)$ & 4 & 0 \\
\hline T2803СC13329T & $2021 / 8 / 8$ & $2021 / 8 / 11$ & $3(3 / 0)$ & 0 & 3 \\
\hline Т2803СС13329TC28313Т & $2021 / 8 / 10$ & $2021 / 8 / 10$ & $1(1 / 0)$ & 0 & 1 \\
\hline T2803CC14573TC14576GC2939T & $2021 / 7 / 27$ & $2021 / 7 / 27$ & $1(0 / 1)$ & 1 & 0 \\
\hline T2803CC14573TC14576GT7513CA27156T & $2021 / 7 / 28$ & $2021 / 7 / 28$ & $1(0 / 1)$ & 1 & 0 \\
\hline T2803CC14573TC14576GT14562C & $2021 / 7 / 26$ & $2021 / 7 / 26$ & $1(0 / 1)$ & 1 & 0 \\
\hline T2803CC14573TC14576GT14562CC23188GC16114TC23300TC25339TG24413T & $2021 / 7 / 28$ & $2021 / 7 / 28$ & $1(0 / 1)$ & 1 & 0 \\
\hline T2803CC14573TC14576GT14562CT7513CC18486TG10533TC23188G & $2021 / 8 / 4$ & $2021 / 8 / 4$ & $1(0 / 1)$ & 1 & 0 \\
\hline T2803CC14573TC14576GT14562CT7513CC18687T & 2021/8/12 & 2021/8/12 & $1(1 / 0)$ & 0 & 1 \\
\hline T2803CC14573TC14576GT14562CT7513CC23188GC11663A & $2021 / 8 / 5$ & $2021 / 8 / 5$ & $1(1 / 0)$ & 1 & 0 \\
\hline T2803СC14708TC14805T & $2021 / 8 / 5$ & $2021 / 8 / 5$ & $1(1 / 0)$ & 1 & 0 \\
\hline T2803CC14805T & $2021 / 8 / 4$ & $2021 / 8 / 12$ & $2(2 / 0)$ & 1 & 1 \\
\hline T2803CC14805TG558T & $2021 / 8 / 13$ & $2021 / 8 / 13$ & $1(1 / 0)$ & 0 & 1 \\
\hline
\end{tabular}


T2803CC14922T

T2803CC14922TC17444T

T2803CC14922TC17444TC21772T

T2803CC14922TC17444TC23525T

T2803CC17336T

T2803CC17410T

T2803CC1758T

T2803CC19263T

T2803CC19488T

T2803CC19955T

T2803CC2106T

T2803CC21575T

T2803CC22712TC7169T

T2803CC23248T

T2803CC23525T

T2803CC23525TC8616T

T2803CC23525TT23248A

T2803CC24912T

T2803CC2523TC2676T

T2803CC25452TC12809TC15222T

T2803CC2571T

T2803CC27641T

T2803CC2939TG27703T

T2803CC29614T

T2803CC29738T

T2803CC4901T

T2803CC5079T

T2803CC5849T

T2803CC9170TG1729A

T2803CG12798A

T2803CG23282T

T2803CG23501T

T2803CG23501TG21372T

\begin{tabular}{|c|c|c|c|c|}
\hline $2021 / 8 / 8$ & $2021 / 8 / 8$ & $1(1 / 0)$ & 0 & 1 \\
\hline $2021 / 7 / 31$ & $2021 / 8 / 1$ & $2(0 / 2)$ & 2 & \\
\hline 2021/8/2 & $2021 / 8 / 2$ & $1(1 / 0)$ & 1 & 0 \\
\hline $2021 / 8 / 8$ & $2021 / 8 / 8$ & $1(1 / 0)$ & 0 & 1 \\
\hline $2021 / 8 / 6$ & $2021 / 8 / 10$ & $3(1 / 2)$ & 2 & 1 \\
\hline $2021 / 8 / 2$ & $2021 / 8 / 10$ & $2(1 / 1)$ & 1 & 1 \\
\hline $2021 / 8 / 13$ & $2021 / 8 / 13$ & $4(4 / 0)$ & 0 & 4 \\
\hline $2021 / 8 / 8$ & $2021 / 8 / 8$ & $1(1 / 0)$ & 0 & 1 \\
\hline $2021 / 8 / 5$ & $2021 / 8 / 6$ & $4(0 / 2)$ & 4 & 0 \\
\hline $2021 / 8 / 4$ & $2021 / 8 / 4$ & $1(0 / 1)$ & 1 & 0 \\
\hline $2021 / 8 / 4$ & $2021 / 8 / 12$ & $6(6 / 0)$ & 3 & 3 \\
\hline $2021 / 8 / 5$ & $2021 / 8 / 10$ & $4(4 / 0)$ & 2 & 2 \\
\hline $2021 / 7 / 29$ & $2021 / 7 / 29$ & $1(0 / 1)$ & 1 & 0 \\
\hline $2021 / 8 / 1$ & $2021 / 8 / 10$ & $6(5 / 1)$ & 3 & 3 \\
\hline $2021 / 8 / 2$ & $2021 / 8 / 2$ & $1(0 / 1)$ & 1 & 0 \\
\hline $2021 / 8 / 7$ & $2021 / 8 / 8$ & $2(2 / 0)$ & 1 & 1 \\
\hline $2021 / 8 / 6$ & $2021 / 8 / 6$ & $1(0 / 1)$ & 1 & 0 \\
\hline $2021 / 8 / 5$ & $2021 / 8 / 10$ & $2(1 / 1)$ & 1 & 1 \\
\hline $2021 / 8 / 8$ & $2021 / 8 / 10$ & $2(1 / 1)$ & 0 & 2 \\
\hline $2021 / 8 / 3$ & $2021 / 8 / 3$ & $1(1 / 0)$ & 1 & 0 \\
\hline $2021 / 7 / 30$ & $2021 / 7 / 30$ & $1(1 / 0)$ & 1 & 0 \\
\hline $2021 / 8 / 8$ & $2021 / 8 / 8$ & $1(1 / 0)$ & 0 & 1 \\
\hline $2021 / 8 / 3$ & $2021 / 8 / 3$ & $1(1 / 0)$ & 1 & 0 \\
\hline $2021 / 8 / 13$ & $2021 / 8 / 13$ & $1(1 / 0)$ & 0 & 1 \\
\hline $2021 / 8 / 4$ & $2021 / 8 / 4$ & $1(0 / 1)$ & 1 & 0 \\
\hline $2021 / 8 / 9$ & $2021 / 8 / 9$ & $1(0 / 1)$ & 0 & 1 \\
\hline $2021 / 8 / 7$ & 2021/8/7 & $1(1 / 0)$ & 1 & \\
\hline $2021 / 8 / 3$ & $2021 / 8 / 15$ & $5(3 / 2)$ & 4 & \\
\hline $2021 / 8 / 5$ & $2021 / 8 / 5$ & $1(0 / 1)$ & 1 & 0 \\
\hline $2021 / 8 / 7$ & $2021 / 8 / 7$ & $1(1 / 0)$ & 1 & 0 \\
\hline $2021 / 8 / 2$ & $2021 / 8 / 2$ & $1(0 / 1)$ & 1 & 0 \\
\hline $2021 / 8 / 8$ & $2021 / 8 / 8$ & $1(0 / 1)$ & 0 & 1 \\
\hline $2021 / 8 / 10$ & $2021 / 8 / 10$ & $1(0 / 1)$ & 0 & 1 \\
\hline
\end{tabular}




\begin{tabular}{|c|c|c|c|c|c|}
\hline T2803CG26389T & $2021 / 7 / 30$ & $2021 / 7 / 30$ & $1(1 / 0)$ & 1 & 0 \\
\hline T2803CG26389TG942A & $2021 / 8 / 6$ & $2021 / 8 / 8$ & $2(2 / 0)$ & 1 & 1 \\
\hline T2803CG26951C & $2021 / 8 / 2$ & $2021 / 8 / 2$ & $1(0 / 1)$ & 1 & 0 \\
\hline T2803CG26951CA27010C & $2021 / 8 / 6$ & $2021 / 8 / 6$ & $1(1 / 0)$ & 1 & 0 \\
\hline T2803CG295T & $2021 / 8 / 11$ & $2021 / 8 / 11$ & $1(0 / 1)$ & 0 & 1 \\
\hline T2803CG3109T & $2021 / 8 / 11$ & $2021 / 8 / 11$ & $1(1 / 0)$ & 0 & 1 \\
\hline T2803CG558T & $2021 / 8 / 7$ & $2021 / 8 / 7$ & $1(1 / 0)$ & 1 & 0 \\
\hline T2803CT25518G & $2021 / 8 / 10$ & $2021 / 8 / 10$ & $1(0 / 5)$ & 0 & 1 \\
\hline Т2803СТ7378C & $2021 / 8 / 7$ & $2021 / 8 / 7$ & $1(0 / 1)$ & 1 & 0 \\
\hline Т2803СТ7513C & $2021 / 7 / 24$ & $2021 / 8 / 9$ & $47(28 / 19)$ & 42 & 5 \\
\hline Т2803СТ7513СА27156T & $2021 / 8 / 12$ & $2021 / 8 / 12$ & $1(1 / 0)$ & 0 & 1 \\
\hline T2803CT7513CA5458GC2143T23641АT26972C & $2021 / 8 / 7$ & $2021 / 8 / 7$ & $1(0 / 1)$ & 1 & 0 \\
\hline T2803CT7513CA655G & $2021 / 8 / 5$ & $2021 / 8 / 5$ & $1(1 / 0)$ & 1 & 0 \\
\hline Т2803СT7513СC1758T & $2021 / 8 / 2$ & $2021 / 8 / 2$ & $1(1 / 0)$ & 1 & 0 \\
\hline T2803CT7513CC18486TG10533T & $2021 / 7 / 24$ & $2021 / 8 / 8$ & $8(5 / 3)$ & 7 & 1 \\
\hline T2803CT7513CC18486TG10533TC13176T & $2021 / 8 / 7$ & $2021 / 8 / 7$ & $1(1 / 0)$ & 1 & 0 \\
\hline Т2803СТ7513СC18687T & $2021 / 8 / 5$ & $2021 / 8 / 5$ & $1(1 / 0)$ & 1 & 0 \\
\hline Т2803СТ7513СC22735T & $2021 / 8 / 9$ & $2021 / 8 / 9$ & $1(0 / 1)$ & 0 & 1 \\
\hline Т2803СТ7513CC275A & $2021 / 7 / 30$ & $2021 / 7 / 31$ & $2(2 / 0)$ & 2 & 0 \\
\hline Т2803CT7513СC2947T & $2021 / 7 / 26$ & $2021 / 7 / 26$ & $1(0 / 1)$ & 1 & 0 \\
\hline T2803CT7513CC5147TC5385TG1018A & $2021 / 8 / 13$ & $2021 / 8 / 15$ & $2(2 / 0)$ & 0 & 2 \\
\hline Т2803СТ7513СС6807Т & $2021 / 7 / 26$ & $2021 / 8 / 4$ & $4(3 / 1)$ & 4 & 0 \\
\hline T2803CT7513CG23282TC29358T & $2021 / 8 / 4$ & $2021 / 8 / 7$ & $2(0 / 2)$ & 2 & 0 \\
\hline T2803CT7513CG24577TG25186T & $2021 / 7 / 29$ & $2021 / 8 / 1$ & $4(3 / 1)$ & 4 & 0 \\
\hline T2803CT7513CG29645T & $2021 / 8 / 16$ & $2021 / 8 / 16$ & $1(0 / 1)$ & 0 & 1 \\
\hline T2803CT7513CG29781T & $2021 / 8 / 7$ & $2021 / 8 / 12$ & $5(2 / 1)$ & 2 & 3 \\
\hline Т2803СТ7513СТ694A & $2021 / 8 / 5$ & $2021 / 8 / 5$ & $1(1 / 0)$ & 1 & 0 \\
\hline
\end{tabular}

253

Combination of Mutation: The emergence of Combination of Mutation in Yangzhou.

Time of occurrence of first case: Date of the earliest case among the cases with the Combination of Mutation.

Time of occurrence of last case: Date of the last case among the cases with the Combination of Mutation.

Total number of cases (controlled/uncontrolled): total number of cases of this mutation combination in Yangzhou.

Controlled number: onset date of cases after NPI measures (include same date). uncontrolled number: onset date of cases before NPI measures).

Number of cases in control period: Number of cases with onset dates in $\mathrm{Cl}$ and ER stage.

Number of cases in uncontrolled period: Number of cases with onset dates in NE and II. 
261 Extended data Table 5 | Summary table of Nanjing-Major combinations of mutations.

\begin{tabular}{|c|c|c|c|}
\hline Nanjing-—Major combinations of mutations & Number of cases & DATE (First case) & DATE (Last case) \\
\hline 35basic mutation (Common) & 51 & $2021 / 7 / 13$ & $2021 / 7 / 31$ \\
\hline 35basic mutation+1 iSNV(Ex346/2803)(Rare) & 12 & $2021 / 7 / 20$ & $2021 / 8 / 2$ \\
\hline 35basic mutation+2 iSNV(Ex346/2803)(Rare) & 2 & $2021 / 7 / 20$ & $2021 / 7 / 30$ \\
\hline 35basic mutation+3 iSNV(Ex346/2803)(Rare) & 1 & $2021 / 8 / 11$ & $2021 / 8 / 11$ \\
\hline 35basic mutation+4 iSNV(Ex346/2803)(Rare) & 1 & $2021 / 7 / 26$ & $2021 / 7 / 26$ \\
\hline 35basic mutation +1 iSNV(Ex346/2803)(Common) & 16 & $2021 / 7 / 16$ & $2021 / 8 / 1$ \\
\hline 35basic mutation+2 iSNV(Ex346/2803)(Common) & 6 & $2021 / 7 / 20$ & $2021 / 8 / 8$ \\
\hline 35basic mutation+C346T(Common) & 14 & $2021 / 7 / 13$ & $2021 / 7 / 27$ \\
\hline 35basic mutation+C346T +1 iSNV(Ex2803)(Rare) & 3 & $2021 / 7 / 19$ & $2021 / 7 / 23$ \\
\hline 35basic mutation+C346T+2 iSNV(Rare) & 4 & $2021 / 7 / 25$ & $2021 / 7 / 29$ \\
\hline 35basic mutation $+\mathrm{C} 346 \mathrm{~T}+3$ iSNV(Ex2803)(Rare) & 1 & $2021 / 7 / 31$ & $2021 / 7 / 31$ \\
\hline 35basic mutation+C346T+C1060T(Common) & 9 & $2021 / 7 / 20$ & $2021 / 7 / 25$ \\
\hline 35basic mutation+C346T+C8772T(Common) & 6 & $2021 / 7 / 19$ & $2021 / 7 / 27$ \\
\hline 35basic mutation $+\mathrm{C} 346 \mathrm{~T}+\mathrm{G} 28209 \mathrm{~T}(\mathrm{Common})$ & 2 & $2021 / 7 / 19$ & $2021 / 7 / 25$ \\
\hline 35basic mutation $+\mathrm{C} 346 \mathrm{~T}+\mathrm{G} 11083 \mathrm{~T}(\mathrm{Common})$ & 5 & $2021 / 7 / 20$ & $2021 / 7 / 26$ \\
\hline 35basic mutation+C346T+A25934C(Common) & 3 & $2021 / 7 / 22$ & $2021 / 7 / 29$ \\
\hline 35basic mutation+C346T+A6411G(Common) & 2 & $2021 / 7 / 20$ & $2021 / 7 / 23$ \\
\hline 35basic mutation+C346T $+\mathrm{G} 28209 \mathrm{~T}+\mathrm{A} 25934 \mathrm{C}+\mathrm{C} 17822 \mathrm{~T}(\mathrm{Common})$ & 2 & $2021 / 8 / 3$ & $2021 / 8 / 5$ \\
\hline 35basic mutation+T2803C(Common) & 19 & $2021 / 7 / 29$ & $2021 / 7 / 31$ \\
\hline 35basic mutation $+\mathrm{T} 2803 \mathrm{C}+1$ iSNV(Ex346)(Rare) & 2 & $2021 / 7 / 26$ & $2021 / 7 / 28$ \\
\hline 35basic mutation $+\mathrm{T} 2803 \mathrm{C}+3$ iSNV(Ex346)(Rare) & 1 & $2021 / 7 / 30$ & $2021 / 7 / 30$ \\
\hline 35basic mutation+T2803C+6 iSNV(Ex346)(Rare) & 1 & $2021 / 7 / 21$ & $2021 / 7 / 21$ \\
\hline 35basic mutation+T2803C+G16968T(Common) & 2 & $2021 / 7 / 28$ & $2021 / 8 / 2$ \\
\hline 35basic mutation $+\mathrm{T} 2803 \mathrm{C}+\mathrm{T} 7513 \mathrm{C}$ (Common) & 2 & $2021 / 7 / 29$ & $2021 / 8 / 10$ \\
\hline
\end{tabular}

263 Rare: Indicates that the same mutation combination occurs in only one case.

264 Common: Indicates that the same mutation combination occurs in multiple cases.

DATE (Last case) : is the onset time of the last case of this mutation combination. 
268 Extended data Table 6 | Summary table of Yangzhou-Major combinations of mutations.

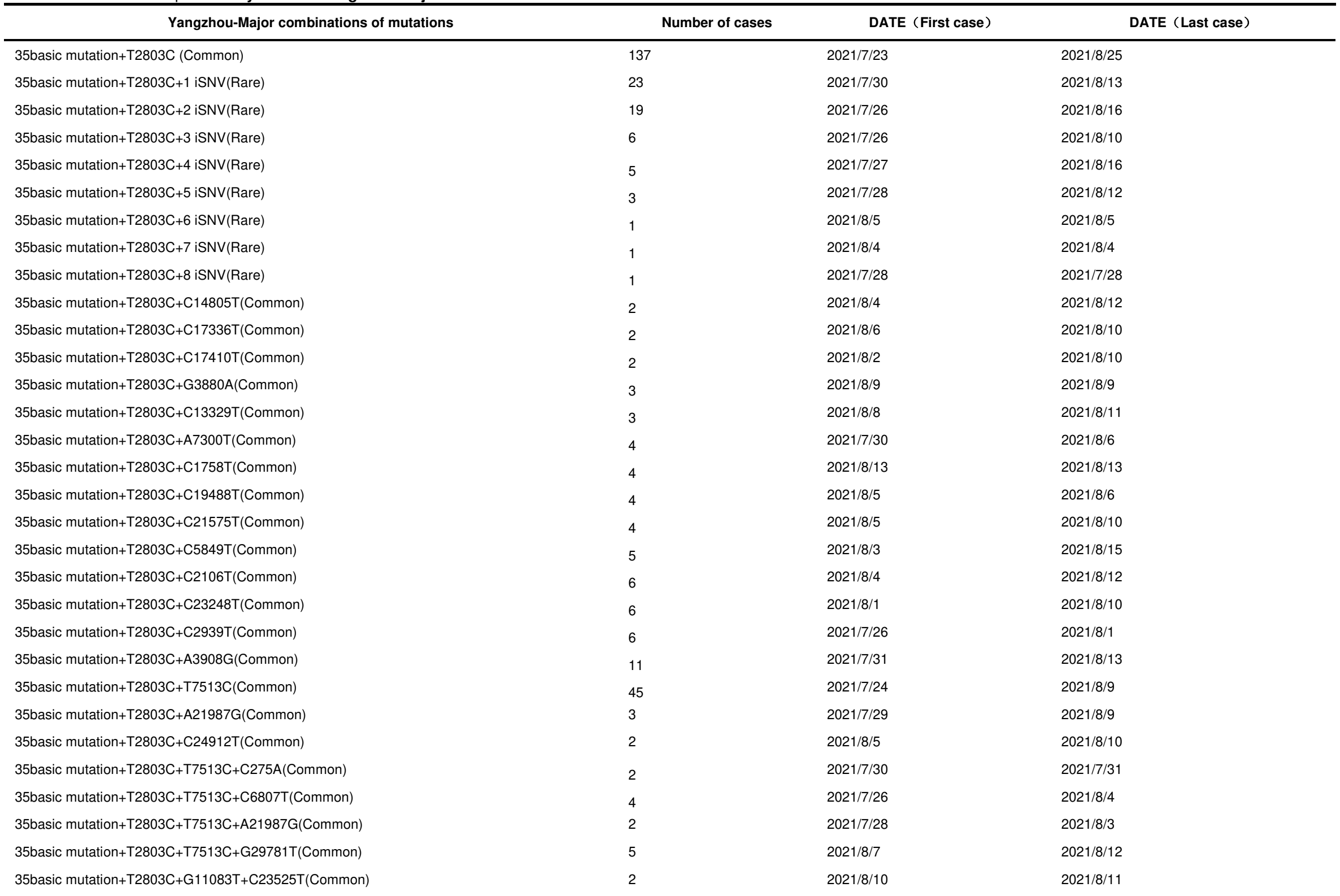


35basic mutation+T2803C+A15201G+G26951C(Common)

35basic mutation+T2803C+A3908G+G4399T(Common)

35basic mutation $+\mathrm{T} 2803 \mathrm{C}+\mathrm{C} 14922 \mathrm{~T}+\mathrm{C} 17444 \mathrm{~T}$ (Common)

35basic mutation $+\mathrm{T} 2803 \mathrm{C}+\mathrm{C} 23525 \mathrm{~T}+\mathrm{C} 8616 \mathrm{~T}$ (Common)

35basic mutation+T2803C+C2523T+C2676T(Common)

35basic mutation+T2803C+G26389T+G942A(Common)

35basic mutation+T2803C+T7513C+C18486T+G10533T(Common)

35basic mutation+T2803C+T7513C+G23282T+C29358T(Common)

35basic mutation+T2803C+T7513C+G24577T+G25186T(Common)

35basic mutation $+\mathrm{T} 2803 \mathrm{C}+\mathrm{T} 7513 \mathrm{C}+\mathrm{C} 5147 \mathrm{~T}+\mathrm{C} 5385 \mathrm{~T}+\mathrm{G} 1018 \mathrm{~A}(\mathrm{Common})$
$2021 / 8 / 5$

$2021 / 8 / 4$

$2021 / 7 / 31$

$2021 / 8 / 7$

$2021 / 8 / 8$

$2021 / 8 / 6$

$2021 / 7 / 24$

$2021 / 8 / 4$

$2021 / 7 / 29$

$2021 / 8 / 13$
$2021 / 8 / 13$

$2021 / 8 / 7$

$2021 / 8 / 1$

$2021 / 8 / 8$

$2021 / 8 / 10$

$2021 / 8 / 8$

$2021 / 8 / 8$

$2021 / 8 / 7$

$2021 / 8 / 1$

$2021 / 8 / 15$

Rare: Indicates that the same mutation combination occurs in only one case.

Common: Indicates that the same mutation combination occurs in multiple cases.

DATE (First case): is the onset time of the First case of this mutation combination.

DATE (Last case): is the onset time of the last case of this mutation combination. 
275 Extended data Table 7 | SNV combinations can be terminated by NPI and vaccine.

\begin{tabular}{|c|c|c|c|c|c|c|}
\hline \multirow[t]{2}{*}{ Variables } & \multirow{2}{*}{$\begin{array}{l}\text { Non-Last } \\
\text { Patient(case/total) } \\
\mathrm{n}=371\end{array}$} & \multirow{2}{*}{$\begin{array}{l}\text { Last } \\
\text { Patient(case/total) } \\
\mathrm{n}=149\end{array}$} & \multirow{2}{*}{$\begin{array}{l}\text { Univariable analysis } \\
\text { Odds ratio }(95 \% \mathrm{Cl})\end{array}$} & \multicolumn{3}{|c|}{ Multivariable analysis } \\
\hline & & & & $p$ value & Odds ratio $(95 \% \mathrm{Cl})$ & $p$ value \\
\hline \multicolumn{7}{|l|}{ Onset Period } \\
\hline NE & $90(24.3 \%)$ & $13(8.7 \%)$ & Ref & & Ref & .. \\
\hline ॥ & $188(50.7 \%)$ & $62(41.6 \%)$ & $2.283(1.194-4.367)$ & 0.013 & $2.467(1.277-4.764)$ & 0.007 \\
\hline $\mathrm{Cl}$ & $90(24.3 \%)$ & $67(45 \%)$ & $5.154(2.659-9.991)$ & $<0.001$ & $5.774(2.931-11.374)$ & $<0.001$ \\
\hline ER & $3(0.8 \%)$ & $7(4.7 \%)$ & $16.154(3.706-70.413)$ & $<0.001$ & $16.118(3.616-71.836)$ & $<0.001$ \\
\hline \multicolumn{7}{|l|}{ Vaccination } \\
\hline No & $235(63.3 \%)$ & $83(55.7 \%)$ & Ref & .. & Ref &. \\
\hline Vaccined $<30$ days & $98(26.4 \%)$ & $40(26.8 \%)$ & $1.156(0.741-1.803)$ & 0.524 & $1.113(0.701-1.769)$ & 0.649 \\
\hline Vaccined $\geq 30$ days & $38(10.2 \%)$ & $26(17.4 \%)$ & $1.937(1.109-3.385)$ & 0.02 & $2.33(1.281-4.236)$ & 0.006 \\
\hline \multicolumn{7}{|l|}{ Age } \\
\hline$<18$ & $48(12.9 \%)$ & $21(14.1 \%)$ & Ref & .. & .. & .. \\
\hline$[18-30)$ & $32(8.6 \%)$ & $12(8.1 \%)$ & $0.857(0.371-1.983)$ & 0.719 & $\mathrm{Nl} \dagger$ & \\
\hline$[30-40)$ & $44(11.9 \%)$ & $22(14.8 \%)$ & $1.143(0.554-2.358)$ & 0.718 & $\mathrm{Nl}+$ & \\
\hline$[40-50)$ & $55(14.8 \%)$ & $32(21.5 \%)$ & $1.33(0.679-2.607)$ & 0.406 & $\mathrm{NI} \dagger$ & \\
\hline$[50-60)$ & $66(17.8 \%)$ & $26(17.4 \%)$ & $0.9(0.454-1.786)$ & 0.764 & $\mathrm{Nl} \dagger$ & \\
\hline$\geq 60$ & $126(34 \%)$ & $36(24.2)$ & $0.653(0.347-1.229)$ & 0.187 & $\mathrm{NIt}$ & \\
\hline \multicolumn{7}{|l|}{ Sex } \\
\hline Woman & $220(59.3 \%)$ & $77(51.7 \%)$ & Ref & .. & .. & .. \\
\hline Man & $151(40.7 \%)$ & $72(48.3 \%)$ & $1.362(0.93-1.997)$ & 0.113 & $\mathrm{Nl}+$ & \\
\hline \multicolumn{7}{|l|}{ Ст } \\
\hline$<20$ & $72(19.4 \%)$ & $36(24.2 \%)$ & Ref & & .. & \\
\hline$[20-30)$ & $194(52.3 \%)$ & $74(49.7 \%)$ & $0.763(0.471-1.235)$ & 0.271 & $\mathrm{Nl}+$ & \\
\hline$\geq 30$ & $105(28.3 \%)$ & $39(26.2 \%)$ & $0.743(0.431-1.279)$ & 0.284 & $\mathrm{Nl} \dagger$ & \\
\hline
\end{tabular}




\section{Clustering}

Nonclustered

149(40.2\%)

$66(44.3 \%)$

Ref

Clustered

222(59.8\%)

$83(55.7 \%)$

$0.844(0.575-1.239)$

0.387

$\mathrm{NIt}$

\section{Clinical}

Mild

\section{Moderate}

71(19.1\%)

$31(20.8 \%)$

$269(72.5 \%)$

114(76.5\%)

Severe+Critica

$31(8.4 \%)$

$4(2.7 \%)$

Ref

Termination codon

No

$367(98.9 \%)$

145(97.3)

$4(1.1 \%)$

$4(2.7 \%)$

Ref

0.971(0.603-1.561)

0.902

0.296(0.096-0.909)

$\mathrm{NIT}$

$0.033 \quad \mathrm{NI}$

2.531(0.625-10.255)

$\mathrm{NI}+$

$\mathrm{NIt}$

276

Univariable and multivariable analysis of factors affecting the termination of iSNV mutation combinations.

Vaccination: $\mathrm{No}=$ Unvaccinated persons were defined as those who had not been vaccinated and those who had less than 14 days between the time of last vaccination and the time of illness.

Yes = Vaccinations were defined as those who were vaccinated and the interval between the last dose of vaccination and the onset of illness was 14 days or more.

$280 \mathrm{NI} \dagger=$ These factors were not included in the multivariable analysis. 
282 Extended data Table 8 | Demographic characteristics of COVID-19 cases in Nanjing and Yangzhou.

\begin{tabular}{|c|c|c|c|}
\hline \multicolumn{2}{|c|}{ Demographic data } & \multirow{2}{*}{$\begin{array}{c}\begin{array}{c}\text { Cases in Nanjing } \\
(\mathrm{n}=221)\end{array} \\
42(19.00 \%)\end{array}$} & \multirow{2}{*}{$\begin{array}{c}\begin{array}{c}\text { Cases in Yangzhou } \\
(\mathrm{n}=559)\end{array} \\
125(22.36 \%)\end{array}$} \\
\hline Age & $<30$ & & \\
\hline & {$[30-40)$} & $34(15.38 \%)$ & 65 (11.63\%) \\
\hline & {$[40-50)$} & $68(30.77 \%)$ & 57 (10.20\%) \\
\hline & {$[50-60)$} & $48(21.72 \%)$ & 87 (15.56\%) \\
\hline & $\geq 60$ & 29 (13.12\%) & 225 (40.25\%) \\
\hline \multirow[t]{2}{*}{ Sex } & Man & $85(38.50 \%)$ & $237(42.40 \%)$ \\
\hline & Woman & 136 (61.50\%) & 322 (57.60\%) \\
\hline \multirow[t]{4}{*}{ CT Value } & $<20$ & 57 (25.79\%) & 91 (16.28\%) \\
\hline & {$[20-30)$} & $100(45.25 \%)$ & 276 (49.37\%) \\
\hline & $\geq 30$ & $51(23.08 \%)$ & $190(33.99 \%)$ \\
\hline & unknown & $13(5.88 \%)$ & $2(0.36 \%)$ \\
\hline \multirow[t]{2}{*}{ Clustering } & Nonclustered & $189(85.52 \%)$ & $109(19.50 \%)$ \\
\hline & Clustered & $32(14.48 \%)$ & $450(80.50 \%)$ \\
\hline \multirow[t]{3}{*}{ Vaccination } & No & 105 (47.51\%) & $379(67.80 \%)$ \\
\hline & Vaccined $<30$ days & $16(7.24 \%)$ & $38(6.80 \%)$ \\
\hline & Vaccined $\geq 30$ days & 100 (45.25\%) & $142(25.40 \%)$ \\
\hline \multirow[t]{4}{*}{ Clinical type } & Mild & $160(72.40 \%)$ & $419(74.96 \%)$ \\
\hline & Moderate & $51(23.08 \%)$ & 85 (15.21\%) \\
\hline & Severe & $1(0.45 \%)$ & 19 (3.40\%) \\
\hline & Critical & $9(4.07 \%)$ & 36 (6.44\%) \\
\hline
\end{tabular}


Extended data Table 9 | Key reagents.

\begin{tabular}{|c|c|c|}
\hline REAGENT & SOURCE & IDENTIFIER \\
\hline SuperScript ${ }^{\mathrm{TM}}$ IV First-Strand Synthesis System & Thermo Fisher,USA & Cat\# 18091050 \\
\hline Q5® Hot Start High-Fidelity 2X Master Mix & NEB,USA & Cat\# M0494S \\
\hline Long Fragment Target Capture Kit for SARS-CoV-2 Whole Genome & BAIYITECH,China & Cat\# BK-LFWCoV024 \\
\hline Target Capture Kit for SARS-CoV-2 Whole Genome & BAIYITECH,China & Cat\# BK-WCoV024 \\
\hline TruePrep DNA Library Prep Kit V2 for Illumina & Vazyme,China & Cat\# TD502-02 \\
\hline TruePrep index Kit V2 for Illumina & Vazyme,China & Cat\# TD202 \\
\hline PhiX Control v3 Support & Illumina,USA & Cat\# FC-110-3001 \\
\hline MiSeq ${ }^{\mathrm{TM}}$ Reagent Kit v2 (300-cycles) & Illumina,USA & Cat\# MS-102-2002 \\
\hline ATOPlex RNA Multiplex PCR-based Library Preparation Set V3.0 & MGI,China & Cat\# 940-000133-00 \\
\hline MGIEasy Fast PCR-FREE FS Library Prep Set & MGI,China & Cat\# 940-000021-00 \\
\hline DNBSEQ one-step & MGI,China & Cat\# 1000026466 \\
\hline MGISEQ-2000RS High-throughput Sequencing Set (FCS SE100) & MGI,China & Cat\# 1000020570 \\
\hline CPAS Barcode Primer 4 Reagent Kit & MGI,China & Cat\# 1000014048 \\
\hline Agilent High Sensitivity DNA Kit & Agilent,USA & Cat\# 5067-4626 \\
\hline NGS Reverse Transcription Kit & ABI,USA & Cat\# A45003 \\
\hline Ion AmpliSeq SARS-CoV-2 Insight Research Assay - GS Chef-Ready & ABI,USA & Cat\# A51306 \\
\hline Ion Torrent ${ }^{\mathrm{TM}}$ Ion Library TaqMan ${ }^{\mathrm{TM}}$ Quantitation Kit & ABI,USA & Cat\# 4468802 \\
\hline Ion $530^{\text {TM }}$ Chip Kit $(2 \times 4$-pack) & ABI,USA & Cat\# A27764 \\
\hline $\begin{array}{l}\text { Ion } 510^{\mathrm{TM}} \& \text { lon } 520^{\mathrm{TM}} \& \text { lon } 530^{\mathrm{TM}} \mathrm{Kit}-\text { Chef }(200 \mathrm{bp}, 2 \text { sequencing run per } \\
\text { initialization) }\end{array}$ & ABI,USA & Cat\# A34461 \\
\hline High Pure PCR Product Purification Kit & Roche,New Zealand & Cat\# 11732668001 \\
\hline Agencourt AMPure XP & Beckman,USA & Cat\# A63881 \\
\hline Qubit $^{\mathrm{TM}}$ dsDNA HS Assay Kit & Thermo Fisher,USA & Cat\# Q32851 \\
\hline Qubit ${ }^{\mathrm{TM}}$ dsDNA BR Assay Kit & Thermo Fisher,USA & Cat\# Q32850 \\
\hline Qubit RNA HS Assay Kit & Thermo Fisher,USA & Cat\# Q32852 \\
\hline ULSEN Ultra-sensitive Novel Coronavirus Whole-genome capture Kit & MicroFuture,China & Cat\# V-090418-1 \\
\hline Nextera DNA Flex Library Prep & Illumina,USA & Cat\# 20018704 \\
\hline NXTR $\otimes$ XT DNA SMP Prep Kit & Illumina,USA & Cat\# 15032785 \\
\hline
\end{tabular}




\begin{tabular}{lll}
\hline IDT® for Illumina DNA/RNA Unique Dual Indexes Set B & Illumina,USA & Cat\# 20027214 \\
IDT® for Illumina DNA/RNA Unique Dual Indexes Set D & Illumina,USA & Cat\#20027216 \\
NextSeq2000 P2 reagent (300 cycle) & Illumina,USA & Cat\# 20046813 \\
iseq100 Reagent V2 (300 cycle) & Illumina,USA & Cat\# 20031371 \\
MiniSeq Mid Output Kit (300-cycles) & Illumina,USA & Cat\# 15073757 \\
Nextera DNA CD Indexes (24 Indexes) & Illumina,USA & Cat\# 20018707 \\
\hline
\end{tabular}

286 
288 Extended data Table 10 | Sample and sequencing information. Related to Figure 1C.

\begin{tabular}{|c|c|c|c|c|c|c|c|c|c|c|c|c|c|c|}
\hline patient_id & sample_id & $\begin{array}{l}\text { symptom_- } \\
\text { onset_date }\end{array}$ & collection_date & Periods & symptom & $\begin{array}{l}\text { sample_ty } \\
\text { pe }\end{array}$ & $\begin{array}{l}\text { Prefect } \\
\text { ure }\end{array}$ & experiment_type & $\begin{array}{l}\text { sequencing_pla } \\
\text { tform }\end{array}$ & Ct-ORF & $\mathrm{Ct}-\mathrm{N}$ & mapped_reads & coverage & depth \\
\hline NJ001 & NJ001 & $2021 / 7 / 19$ & $2021 / 7 / 21$ & $\mathrm{NE}$ & Mild & OPS & NJ & multiplex-pcr & BGI & 26.9 & 26.1 & $5,893,109$ & $100.00 \%$ & 19715.99 \\
\hline NJ002 & NJ002 & 2021/7/16 & $2021 / 7 / 21$ & $\mathrm{NE}$ & Mild & OPS & NJ & multiplex-pcr & BGI & 20.55 & 19.24 & $5,891,515$ & $100.00 \%$ & 19710.66 \\
\hline NJ003 & NJ003 & $2021 / 7 / 20$ & $2021 / 7 / 21$ & $\mathrm{NE}$ & Moderate & OPS & NJ & multiplex-pcr & Illumina & 27.3 & 26.6 & $1,414,576$ & $99.56 \%$ & 5493.016 \\
\hline NJ004 & NJ004 & $2021 / 7 / 15$ & $2021 / 7 / 21$ & NE & Mild & OPS & NJ & multiplex-pcr & Illumina & 35.4 & 35.3 & $1,021,122$ & $99.94 \%$ & 2583.658 \\
\hline NJ005 & NJ005 & $2021 / 7 / 20$ & $2021 / 7 / 21$ & NE & Moderate & OPS & NJ & multiplex-pcr & Illumina & 33 & 33 & 741,008 & $99.02 \%$ & 1782.179 \\
\hline NJ006 & NJ006 & $2021 / 7 / 17$ & $2021 / 7 / 21$ & $\mathrm{NE}$ & Moderate & OPS & $\mathrm{NJ}$ & multiplex-pcr & Illumina & 30.4 & 28.6 & 765,024 & $99.79 \%$ & 1936.053 \\
\hline NJ007 & NJ007 & $2021 / 7 / 18$ & $2021 / 7 / 21$ & $\mathrm{NE}$ & Moderate & OPS & $\mathrm{NJ}$ & multiplex-pcr & Illumina & 33.3 & 32.1 & 856,100 & $99.41 \%$ & 2360.713 \\
\hline NJ009 & NJ009 & $2021 / 7 / 20$ & $2021 / 7 / 21$ & $\mathrm{NE}$ & Moderate & OPS & $\mathrm{NJ}$ & multiplex-pcr & BGI & 31.4 & 32.1 & $5,395,751$ & $100.00 \%$ & 18052.03 \\
\hline NJ010 & NJ010 & $2021 / 7 / 15$ & $2021 / 7 / 21$ & NE & Mild & OPS & NJ & multiplex-pcr & Illumina & 21 & 20 & 620,017 & $99.94 \%$ & 1597.72 \\
\hline NJ011 & NJ011 & $2021 / 7 / 18$ & $2021 / 7 / 21$ & $\mathrm{NE}$ & Moderate & OPS & $\mathrm{NJ}$ & multiplex-pcr & Illumina & 14 & 12 & 566,054 & $99.94 \%$ & 1478.998 \\
\hline NJ012 & NJ012 & $2021 / 7 / 18$ & $2021 / 7 / 21$ & NE & Moderate & OPS & NJ & multiplex-pcr & Illumina & 18 & 19 & $1,040,647$ & $99.77 \%$ & 4054.291 \\
\hline NJ013 & NJ013 & $2021 / 7 / 20$ & $2021 / 7 / 21$ & NE & Moderate & OPS & NJ & multiplex-pcr & BGI & 31.6 & 28.3 & $1,906,595$ & $100.00 \%$ & 6359.526 \\
\hline NJ014 & NJ014 & $2021 / 7 / 19$ & $2021 / 7 / 21$ & $\mathrm{NE}$ & Mild & OPS & NJ & multiplex-pcr & BGI & 32 & 34 & $5,908,269$ & $100.00 \%$ & 19766.71 \\
\hline NJ015 & NJ015 & $2021 / 7 / 20$ & $2021 / 7 / 21$ & $\mathrm{NE}$ & Moderate & OPS & $\mathrm{NJ}$ & multiplex-pcr & Illumina & 18 & 16 & 458,247 & $99.91 \%$ & 1205.087 \\
\hline NJ020 & $\mathrm{NJ} 020$ & $2021 / 7 / 13$ & $2021 / 7 / 23$ & NE & Mild & OPS & NJ & multiplex-pcr & Illumina & 14 & 16 & $1,491,423$ & $99.94 \%$ & 4594.292 \\
\hline NJ021 & NJ021 & $2021 / 7 / 20$ & $2021 / 7 / 23$ & $\mathrm{NE}$ & Mild & OPS & NJ & multiplex-pcr & Illumina & 26 & 26 & $1,467,781$ & $99.84 \%$ & 4613.42 \\
\hline NJ022 & NJ022 & $2021 / 7 / 20$ & $2021 / 7 / 23$ & $\mathrm{NE}$ & Moderate & OPS & $\mathrm{NJ}$ & multiplex-pcr & Illumina & 20 & 22 & $1,558,870$ & $99.93 \%$ & 4787.205 \\
\hline NJ023 & $\mathrm{NJ} 023$ & $2021 / 7 / 21$ & $2021 / 7 / 23$ & $\mathrm{NE}$ & Moderate & OPS & NJ & multiplex-pcr & Illumina & 31 & 31 & $1,070,088$ & $99.43 \%$ & 3439.416 \\
\hline NJ026 & NJ026 & $2021 / 7 / 20$ & $2021 / 7 / 23$ & $\mathrm{NE}$ & Moderate & OPS & $\mathrm{NJ}$ & multiplex-pcr & BGI & 30 & 30 & $2,991,657$ & $99.90 \%$ & 9992.143 \\
\hline NJ027 & NJ027 & $2021 / 7 / 20$ & $2021 / 7 / 23$ & $\mathrm{NE}$ & Moderate & OPS & $\mathrm{NJ}$ & multiplex-pcr & Illumina & 24 & 24 & $1,903,657$ & $99.84 \%$ & 5739.518 \\
\hline NJ028 & NJ028 & $2021 / 7 / 20$ & $2021 / 7 / 23$ & $\mathrm{NE}$ & Moderate & OPS & $\mathrm{NJ}$ & multiplex-pcr & BGI & 33 & 33 & $1,199,864$ & $99.87 \%$ & 2009.471 \\
\hline NJ031 & NJ031 & $2021 / 7 / 16$ & $2021 / 7 / 21$ & $\mathrm{NE}$ & Moderate & OPS & $\mathrm{NJ}$ & multiplex-pcr & Illumina & 33 & 30 & $2,167,484$ & $99.79 \%$ & 6598.661 \\
\hline NJ032 & NJ032 & $2021 / 7 / 13$ & $2021 / 7 / 21$ & NE & Moderate & OPS & NJ & multiplex-pcr & Illumina & 29 & 26 & $2,073,507$ & $99.65 \%$ & 6357.679 \\
\hline NJ033 & NJ033 & $2021 / 7 / 19$ & $2021 / 7 / 23$ & $\mathrm{NE}$ & Mild & OPS & NJ & multiplex-pcr & Illumina & 22 & 22 & $1,348,934$ & $99.93 \%$ & 4229.287 \\
\hline NJ034 & NJ034 & $2021 / 7 / 19$ & $2021 / 7 / 23$ & $\mathrm{NE}$ & Moderate & OPS & $\mathrm{NJ}$ & multiplex-pcr & BGI & 28 & 28 & $2,622,422$ & $99.88 \%$ & 4391.188 \\
\hline NJ035 & NJ035 & $2021 / 7 / 15$ & $2021 / 7 / 23$ & NE & Moderate & OPS & NJ & multiplex-pcr & BGI & 30 & 31 & $1,929,804$ & $99.90 \%$ & 3230.667 \\
\hline NJ036 & NJ036 & $2021 / 7 / 19$ & $2021 / 7 / 23$ & NE & Moderate & OPS & $\mathrm{NJ}$ & multiplex-pcr & Illumina & 19 & 20 & $1,108,812$ & $99.89 \%$ & 3555.446 \\
\hline
\end{tabular}




\begin{tabular}{|c|c|c|c|c|c|c|c|c|c|c|c|c|c|c|}
\hline NJ037 & NJ037 & $2021 / 7 / 17$ & $2021 / 7 / 23$ & $\mathrm{NE}$ & Moderate & OPS & NJ & multiplex-pcr & Illumina & 28 & 30 & $1,279,287$ & $99.72 \%$ & 4013.909 \\
\hline NJ039 & NJ039 & $2021 / 7 / 22$ & $2021 / 7 / 23$ & $\mathrm{NE}$ & Moderate & OPS & NJ & multiplex-pcr & Illumina & 27 & 28 & $1,313,571$ & $99.83 \%$ & 4214.173 \\
\hline NJ040 & NJ040 & $2021 / 7 / 19$ & $2021 / 7 / 23$ & $\mathrm{NE}$ & Moderate & OPS & NJ & multiplex-pcr & Illumina & 18 & 18 & $1,107,193$ & $99.94 \%$ & 3582.698 \\
\hline NJ041 & NJ041 & $2021 / 7 / 21$ & $2021 / 7 / 23$ & $\mathrm{NE}$ & Moderate & OPS & NJ & multiplex-pcr & Illumina & 25 & 25 & $1,347,369$ & $99.92 \%$ & 4295.82 \\
\hline NJ042 & $\mathrm{NJ} 042$ & $2021 / 7 / 21$ & $2021 / 7 / 23$ & $\mathrm{NE}$ & Mild & OPS & NJ & multiplex-pcr & Illumina & 34 & 34 & $21,798,793$ & $99.94 \%$ & 67072.8 \\
\hline NJ043 & NJ043 & $2021 / 7 / 21$ & $2021 / 7 / 23$ & $\mathrm{NE}$ & Moderate & OPS & NJ & multiplex-pcr & Illumina & 34 & 34 & $4,516,308$ & $99.70 \%$ & 13911.44 \\
\hline NJ044 & NJ044 & $2021 / 7 / 21$ & $2021 / 7 / 23$ & $\mathrm{NE}$ & Moderate & OPS & NJ & multiplex-pcr & Illumina & 17 & 18 & $3,198,293$ & $99.87 \%$ & 9750.579 \\
\hline NJ045 & NJ045 & $2021 / 7 / 21$ & $2021 / 7 / 23$ & $\mathrm{NE}$ & Moderate & OPS & NJ & multiplex-pcr & BGI & 18 & 21 & $3,572,903$ & $99.90 \%$ & 11934.04 \\
\hline NJ046 & NJ046 & $2021 / 7 / 22$ & $2021 / 7 / 23$ & $\mathrm{NE}$ & Moderate & OPS & NJ & multiplex-pcr & Illumina & 29 & 29 & $1,491,545$ & $99.75 \%$ & 4432.82 \\
\hline NJ047 & NJ047 & $2021 / 7 / 21$ & $2021 / 7 / 23$ & $\mathrm{NE}$ & Moderate & OPS & NJ & multiplex-pcr & Illumina & 25 & 25 & $1,133,726$ & $99.70 \%$ & 3595.7 \\
\hline NJ048 & NJ048 & $2021 / 7 / 21$ & $2021 / 7 / 23$ & $\mathrm{NE}$ & Moderate & OPS & NJ & multiplex-pcr & Illumina & 27 & 28 & $2,209,793$ & $99.89 \%$ & 6822.537 \\
\hline NJ049 & NJ049 & $2021 / 7 / 16$ & $2021 / 7 / 23$ & $\mathrm{NE}$ & Moderate & OPS & NJ & multiplex-pcr & Illumina & 22 & 23 & 840,602 & $99.84 \%$ & 2390.311 \\
\hline NJ050 & NJ050 & $2021 / 7 / 19$ & $2021 / 7 / 23$ & $\mathrm{NE}$ & Moderate & OPS & NJ & multiplex-pcr & Illumina & 22 & 24 & $1,007,111$ & $99.74 \%$ & 2937.674 \\
\hline NJ051 & NJ051 & $2021 / 7 / 21$ & $2021 / 7 / 23$ & $\mathrm{NE}$ & Moderate & OPS & NJ & multiplex-pcr & Illumina & 22 & 24 & $1,077,943$ & $99.77 \%$ & 3129.545 \\
\hline NJ052 & NJ052 & $2021 / 7 / 19$ & $2021 / 7 / 24$ & $\mathrm{NE}$ & Moderate & OPS & NJ & multiplex-pcr & Illumina & 20 & 21 & 824,837 & $99.93 \%$ & 2389.559 \\
\hline NJ053 & $\mathrm{NJ} 053$ & $2021 / 7 / 21$ & $2021 / 7 / 24$ & II & Moderate & OPS & NJ & multiplex-pcr & Illumina & 22 & 22 & $1,184,305$ & $99.94 \%$ & 3440.324 \\
\hline NJ054 & NJ054 & $2021 / 7 / 23$ & $2021 / 7 / 25$ & II & Moderate & OPS & $\mathrm{NJ}$ & multiplex-pcr & Illumina & 28 & 28 & $56,997,748$ & $99.91 \%$ & 172081.2 \\
\hline NJ055 & NJ055 & $2021 / 7 / 23$ & $2021 / 7 / 25$ & II & Moderate & OPS & NJ & multiplex-pcr & Illumina & 26 & 26 & $62,093,487$ & $99.83 \%$ & 188204 \\
\hline NJ056 & NJ056 & $2021 / 7 / 22$ & $2021 / 7 / 25$ & II & Moderate & OPS & NJ & multiplex-pcr & Illumina & 25 & 25 & $72,371,458$ & $99.91 \%$ & 218513.8 \\
\hline NJ058 & NJ058 & $2021 / 7 / 23$ & $2021 / 7 / 25$ & II & Moderate & OPS & NJ & multiplex-pcr & Illumina & 26 & 25 & $50,849,240$ & $99.91 \%$ & 154657.7 \\
\hline NJ059 & NJ059 & $2021 / 7 / 18$ & $2021 / 7 / 25$ & NE & Moderate & OPS & NJ & multiplex-pcr & Illumina & 26 & 26 & $46,140,680$ & $99.94 \%$ & 140927.6 \\
\hline NJ061 & NJ061 & $2021 / 7 / 22$ & $2021 / 7 / 25$ & II & Moderate & OPS & NJ & multiplex-pcr & Illumina & 28 & 28 & $64,503,228$ & $99.93 \%$ & 194482.5 \\
\hline NJ062 & NJ062 & $2021 / 7 / 23$ & $2021 / 7 / 25$ & II & Moderate & OPS & NJ & multiplex-pcr & Illumina & 14 & 14 & $49,430,285$ & $99.94 \%$ & 151133.8 \\
\hline NJ063 & NJ063 & $2021 / 7 / 24$ & $2021 / 7 / 25$ & II & Moderate & OPS & NJ & multiplex-pcr & Illumina & 19 & 19 & $76,804,846$ & $99.94 \%$ & 232420.8 \\
\hline NJ064 & NJ064 & $2021 / 7 / 24$ & $2021 / 7 / 25$ & II & Moderate & OPS & NJ & multiplex-pcr & Illumina & 22 & 22 & $59,360,876$ & $99.94 \%$ & 180672.5 \\
\hline NJ065 & NJ065 & $2021 / 7 / 23$ & $2021 / 7 / 25$ & II & Mild & OPS & NJ & multiplex-pcr & Illumina & 26 & 26 & $64,107,364$ & $99.94 \%$ & 194675.3 \\
\hline NJ066 & NJ066 & $2021 / 7 / 23$ & $2021 / 7 / 25$ & II & Moderate & OPS & NJ & multiplex-pcr & Illumina & 16 & 16 & $43,046,948$ & $99.94 \%$ & 132379.2 \\
\hline NJ067 & NJ067 & $2021 / 7 / 22$ & $2021 / 7 / 25$ & II & Moderate & OPS & NJ & multiplex-pcr & Illumina & 20 & 21 & $60,190,823$ & $99.94 \%$ & 183448.2 \\
\hline NJ068 & NJ068 & $2021 / 7 / 22$ & $2021 / 7 / 25$ & II & Moderate & OPS & NJ & multiplex-pcr & Illumina & 20 & 22 & $46,339,293$ & $99.94 \%$ & 140256.7 \\
\hline
\end{tabular}




\begin{tabular}{|c|c|c|c|c|c|c|c|c|c|c|c|c|c|c|}
\hline NJ070 & NJ070 & $2021 / 7 / 22$ & $2021 / 7 / 25$ & II & Moderate & OPS & NJ & multiplex-pcr & Illumina & 18 & 20 & $71,347,296$ & $99.94 \%$ & 216842.6 \\
\hline NJ071 & NJ071 & $2021 / 7 / 24$ & $2021 / 7 / 25$ & II & Moderate & OPS & NJ & multiplex-pcr & Illumina & 17 & 17 & $47,620,887$ & $99.94 \%$ & 145410.3 \\
\hline NJ073 & NJ073 & $2021 / 7 / 22$ & $2021 / 7 / 25$ & II & Moderate & OPS & NJ & multiplex-pcr & Illumina & 33 & 33 & $1,716,750$ & $99.94 \%$ & 5737.751 \\
\hline NJ074 & NJ074 & $2021 / 7 / 22$ & $2021 / 8 / 8$ & $\mathrm{NE}$ & Moderate & OPS & NJ & multiplex-pcr & Illumina & 30 & 30 & 435,812 & $99.65 \%$ & 1766.879 \\
\hline NJ075 & NJ075 & $2021 / 7 / 21$ & $2021 / 7 / 31$ & II & Moderate & OPS & NJ & multiplex-pcr & Illumina & 24 & 26 & $15,848,415$ & $99.91 \%$ & 58279.28 \\
\hline NJ078 & NJ078 & $2021 / 7 / 23$ & $2021 / 7 / 31$ & II & Moderate & OPS & NJ & multiplex-pcr & Illumina & 32 & 34 & $8,925,398$ & $99.88 \%$ & 31245.42 \\
\hline NJ079 & NJ079 & $2021 / 7 / 25$ & $2021 / 7 / 31$ & II & Mild & OPS & NJ & multiplex-pcr & Illumina & 34 & 36 & $10,381,604$ & $99.92 \%$ & 32105.38 \\
\hline NJ080 & NJ080 & $2021 / 7 / 25$ & $2021 / 7 / 31$ & II & Moderate & OPS & NJ & multiplex-pcr & Illumina & 22 & 24 & $26,219,270$ & $99.94 \%$ & 100515 \\
\hline NJ082 & NJ082 & $2021 / 7 / 22$ & $2021 / 7 / 31$ & II & Mild & OPS & NJ & multiplex-pcr & Illumina & 17 & 23 & $16,428,932$ & $99.94 \%$ & 64312.04 \\
\hline NJ084 & NJ084 & $2021 / 7 / 23$ & $2021 / 7 / 31$ & II & Moderate & OPS & NJ & multiplex-pcr & Illumina & 17 & 23 & $28,543,584$ & $99.94 \%$ & 111964.9 \\
\hline NJ085 & NJ085 & $2021 / 7 / 21$ & $2021 / 7 / 31$ & II & Moderate & OPS & NJ & multiplex-pcr & Illumina & 17 & 22 & $18,836,610$ & $99.94 \%$ & 73941.13 \\
\hline NJ086 & NJ086 & $2021 / 7 / 25$ & $2021 / 7 / 31$ & II & Moderate & OPS & NJ & multiplex-pcr & Illumina & 23 & 25 & $32,857,352$ & $99.94 \%$ & 127995.6 \\
\hline NJ087 & NJ087 & $2021 / 7 / 22$ & $2021 / 7 / 31$ & II & Mild & OPS & NJ & multiplex-pcr & Illumina & 19 & 21 & $49,255,674$ & $99.94 \%$ & 190979.2 \\
\hline NJ088 & NJ088 & $2021 / 7 / 25$ & $2021 / 7 / 31$ & II & Moderate & OPS & NJ & multiplex-pcr & Illumina & 15 & 17 & $38,257,880$ & $99.94 \%$ & 150158.5 \\
\hline NJ089 & NJ089 & $2021 / 7 / 23$ & $2021 / 7 / 31$ & II & Mild & OPS & NJ & multiplex-pcr & Illumina & 32 & 23 & $5,358,593$ & $99.88 \%$ & 19284.6 \\
\hline NJ090 & NJ090 & $2021 / 7 / 24$ & $2021 / 7 / 31$ & II & Moderate & OPS & NJ & multiplex-pcr & Illumina & 21 & 25 & $29,846,203$ & $99.94 \%$ & 116494.4 \\
\hline NJ091 & NJ091 & $2021 / 7 / 25$ & $2021 / 7 / 31$ & II & Moderate & OPS & $\mathrm{NJ}$ & multiplex-pcr & Illumina & 21 & 24 & $9,941,793$ & $99.94 \%$ & 39078.62 \\
\hline NJ092 & NJ092 & $2021 / 7 / 24$ & $2021 / 7 / 31$ & II & Mild & OPS & NJ & multiplex-pcr & Illumina & 24 & 27 & $12,788,936$ & $99.94 \%$ & 49801.79 \\
\hline NJ093 & NJ093 & $2021 / 7 / 23$ & $2021 / 7 / 31$ & II & Moderate & OPS & NJ & multiplex-pcr & Illumina & 22 & 24 & $15,893,058$ & $99.94 \%$ & 57446.12 \\
\hline NJ094 & NJ094 & $2021 / 7 / 25$ & $2021 / 7 / 31$ & II & Moderate & OPS & NJ & multiplex-pcr & Illumina & 22 & 22 & $8,984,801$ & $99.94 \%$ & 34554.35 \\
\hline NJ095 & NJ095 & $2021 / 7 / 25$ & $2021 / 7 / 31$ & II & Mild & OPS & NJ & multiplex-pcr & Illumina & 16 & 18 & $15,173,624$ & $99.94 \%$ & 58000.42 \\
\hline NJ096 & NJ096 & $2021 / 7 / 25$ & $2021 / 7 / 31$ & II & Moderate & OPS & NJ & multiplex-pcr & Illumina & 14 & 17 & $9,733,624$ & $99.94 \%$ & 36982.72 \\
\hline NJ097 & NJ097 & $2021 / 7 / 24$ & $2021 / 7 / 31$ & II & Moderate & OPS & NJ & multiplex-pcr & Illumina & 23 & 24 & $11,363,700$ & $99.94 \%$ & 42677.63 \\
\hline NJ098 & NJ098 & $2021 / 7 / 25$ & $2021 / 7 / 31$ & II & Moderate & OPS & NJ & multiplex-pcr & Illumina & 32 & 34 & $12,737,216$ & $99.93 \%$ & 46090.27 \\
\hline NJ099 & NJ099 & $2021 / 7 / 25$ & $2021 / 7 / 31$ & II & Mild & OPS & NJ & multiplex-pcr & Illumina & 18 & 21 & $10,816,901$ & $99.91 \%$ & 41025.49 \\
\hline NJ100 & NJ100 & $2021 / 7 / 24$ & $2021 / 7 / 31$ & II & Moderate & OPS & NJ & multiplex-pcr & Illumina & 28 & 29 & $23,993,151$ & $99.88 \%$ & 92676.05 \\
\hline NJ101 & NJ101 & $2021 / 7 / 25$ & $2021 / 7 / 31$ & II & Moderate & OPS & NJ & multiplex-pcr & Illumina & 15 & 18 & $9,730,420$ & $99.94 \%$ & 36687.12 \\
\hline NJ102 & NJ102 & $2021 / 7 / 25$ & $2021 / 7 / 31$ & II & Mild & OPS & NJ & multiplex-pcr & Illumina & 17 & 17 & $12,225,834$ & $99.94 \%$ & 47861.34 \\
\hline NJ103 & NJ103 & 2021/7/19 & $2021 / 7 / 26$ & $\mathrm{NE}$ & Moderate & OPS & NJ & multiplex-pcr & BGI & 22 & 22 & $2,618,222$ & $99.94 \%$ & 5256.675 \\
\hline
\end{tabular}




\begin{tabular}{|c|c|c|c|c|c|c|c|c|c|c|c|c|c|c|}
\hline NJ104 & NJ104 & $2021 / 7 / 25$ & $2021 / 7 / 31$ & II & Moderate & OPS & $\mathrm{NJ}$ & multiplex-pcr & Illumina & 19 & 21 & $8,980,819$ & $99.94 \%$ & 34307.58 \\
\hline NJ105 & NJ105 & $2021 / 7 / 25$ & $2021 / 7 / 31$ & II & Moderate & OPS & $\mathrm{NJ}$ & multiplex-pcr & Illumina & 30 & 32 & $15,530,829$ & $99.85 \%$ & 53571.21 \\
\hline NJ106 & NJ106 & $2021 / 7 / 24$ & $2021 / 7 / 31$ & II & Moderate & OPS & $\mathrm{NJ}$ & multiplex-pcr & Illumina & 16 & 16 & $14,215,135$ & $99.94 \%$ & 52387.41 \\
\hline NJ107 & NJ107 & $2021 / 7 / 25$ & $2021 / 7 / 31$ & II & Moderate & OPS & $\mathrm{NJ}$ & multiplex-pcr & Illumina & 16 & 19 & $12,906,137$ & $99.94 \%$ & 47243.8 \\
\hline NJ110 & NJ110 & $2021 / 7 / 25$ & $2021 / 7 / 31$ & II & Mild & OPS & $\mathrm{NJ}$ & multiplex-pcr & Illumina & 31 & 31 & $10,273,633$ & $99.88 \%$ & 30893.7 \\
\hline NJ114 & NJ114 & $2021 / 7 / 24$ & $2021 / 7 / 27$ & II & Mild & OPS & $\mathrm{NJ}$ & multiplex-pcr & Illumina & 20 & 19 & $4,177,539$ & $99.94 \%$ & 11840.62 \\
\hline NJ115 & NJ115 & $2021 / 7 / 26$ & $2021 / 7 / 27$ & II & Mild & OPS & $\mathrm{NJ}$ & multiplex-pcr & Illumina & 34 & 32 & $1,381,603$ & $99.75 \%$ & 3748.584 \\
\hline NJ120 & NJ120 & $2021 / 7 / 24$ & $2021 / 7 / 31$ & II & Moderate & OPS & $\mathrm{NJ}$ & multiplex-pcr & Illumina & 28.8 & 25.9 & 933,030 & $99.55 \%$ & 3555.006 \\
\hline NJ121 & NJ121 & $2021 / 7 / 22$ & $2021 / 7 / 31$ & II & Moderate & OPS & $\mathrm{NJ}$ & multiplex-pcr & BGI & 38.7 & 35.9 & 94,759 & $99.65 \%$ & 316.3816 \\
\hline NJ124 & $\mathrm{NJ} 124$ & $2021 / 7 / 22$ & $2021 / 7 / 31$ & II & Moderate & OPS & $\mathrm{NJ}$ & multiplex-pcr & Illumina & 18.2 & 15.3 & 368,032 & $99.80 \%$ & 12.33765 \\
\hline NJ125 & NJ125 & $2021 / 7 / 23$ & $2021 / 7 / 31$ & II & Moderate & OPS & $\mathrm{NJ}$ & multiplex-pcr & Illumina & 20.4 & 17.5 & 640,625 & $99.61 \%$ & 1929.753 \\
\hline NJ126 & NJ126 & $2021 / 7 / 26$ & $2021 / 7 / 31$ & II & Moderate & OPS & $\mathrm{NJ}$ & multiplex-pcr & Illumina & 15.7 & 13.2 & 347,775 & $99.59 \%$ & 1013.313 \\
\hline NJ127 & NJ127 & $2021 / 7 / 24$ & $2021 / 7 / 31$ & II & Moderate & OPS & $\mathrm{NJ}$ & multiplex-pcr & Illumina & 30 & 29 & $18,652,075$ & $99.94 \%$ & 67441.5 \\
\hline NJ129 & $\mathrm{NJ} 129$ & $2021 / 7 / 26$ & $2021 / 7 / 31$ & II & Moderate & OPS & $\mathrm{NJ}$ & multiplex-pcr & Illumina & 23.4 & 20.7 & $1,265,743$ & $99.52 \%$ & 4981.298 \\
\hline NJ131 & NJ131 & $2021 / 7 / 25$ & $2021 / 7 / 31$ & II & Mild & OPS & $\mathrm{NJ}$ & multiplex-pcr & Illumina & 17.7 & 14.7 & 956,763 & $99.64 \%$ & 2587.101 \\
\hline NJ132 & $\mathrm{NJ} 132$ & $2021 / 7 / 24$ & $2021 / 7 / 31$ & II & Moderate & OPS & $\mathrm{NJ}$ & multiplex-pcr & Illumina & 25 & 25 & $16,835,744$ & $99.94 \%$ & 62428.16 \\
\hline NJ133 & NJ133 & $2021 / 7 / 26$ & $2021 / 7 / 31$ & II & Mild & OPS & $\mathrm{NJ}$ & multiplex-pcr & Illumina & 29 & 29 & $15,810,833$ & $99.94 \%$ & 57493.49 \\
\hline NJ137 & NJ137 & $2021 / 7 / 26$ & $2021 / 8 / 10$ & II & Moderate & OPS & $\mathrm{NJ}$ & multiplex-pcr & Illumina & 26 & 26 & $2,232,338$ & $99.85 \%$ & 7913.897 \\
\hline NJ138 & NJ138 & $2021 / 7 / 26$ & $2021 / 7 / 31$ & II & Moderate & OPS & $\mathrm{NJ}$ & multiplex-pcr & Illumina & 23.1 & 22 & 264,942 & $99.54 \%$ & 1040.55 \\
\hline NJ141 & NJ141 & $2021 / 7 / 26$ & $2021 / 7 / 31$ & II & Moderate & OPS & $\mathrm{NJ}$ & multiplex-pcr & Illumina & 18.8 & 15.6 & 980,602 & $99.52 \%$ & 2828.862 \\
\hline NJ142 & NJ142 & $2021 / 7 / 26$ & $2021 / 7 / 31$ & II & Moderate & OPS & $\mathrm{NJ}$ & multiplex-pcr & Illumina & 31 & 31 & $5,402,126$ & $99.92 \%$ & 8195.749 \\
\hline NJ143 & NJ143 & $2021 / 7 / 26$ & $2021 / 8 / 10$ & II & Mild & OPS & $\mathrm{NJ}$ & multiplex-pcr & $\mathrm{TF}$ & 34 & 34 & 220,922 & $99.73 \%$ & 1464.925 \\
\hline NJ145 & NJ145 & $2021 / 7 / 26$ & $2021 / 7 / 31$ & II & Mild & OPS & $\mathrm{NJ}$ & multiplex-pcr & Illumina & 22.9 & 19.5 & 738,808 & $99.59 \%$ & 2284.6 \\
\hline NJ146 & NJ146 & $2021 / 7 / 25$ & $2021 / 7 / 31$ & II & Moderate & OPS & $\mathrm{NJ}$ & multiplex-pcr & Illumina & 24 & 20.5 & 494,037 & $99.61 \%$ & 1530.478 \\
\hline NJ152 & NJ152 & $2021 / 7 / 26$ & $2021 / 8 / 10$ & II & Moderate & OPS & $\mathrm{NJ}$ & multiplex-pcr & $\mathrm{TF}$ & 25 & 25 & $2,030,437$ & $99.80 \%$ & 13709.94 \\
\hline NJ156 & NJ156 & $2021 / 7 / 26$ & $2021 / 7 / 31$ & II & Moderate & OPS & $\mathrm{NJ}$ & multiplex-pcr & Illumina & 24.8 & 23.4 & 950,238 & $99.64 \%$ & 2868 \\
\hline NJ157 & $\mathrm{NJ} 157$ & $2021 / 7 / 27$ & $2021 / 7 / 31$ & $\mathrm{CI}$ & Moderate & OPS & $\mathrm{NJ}$ & multiplex-pcr & Illumina & 33.5 & 32 & $1,036,888$ & $99.43 \%$ & 3196.759 \\
\hline NJ158 & NJ158 & $2021 / 7 / 25$ & $2021 / 7 / 31$ & II & Moderate & OPS & $\mathrm{NJ}$ & multiplex-pcr & Illumina & 33.3 & 31.5 & 758,709 & $99.46 \%$ & 2321.692 \\
\hline NJ159 & NJ159 & $2021 / 7 / 27$ & $2021 / 7 / 28$ & $\mathrm{CI}$ & Moderate & OPS & $\mathrm{NJ}$ & multiplex-pcr & Illumina & 12 & 13 & $7,972,661$ & $99.94 \%$ & 22378.86 \\
\hline
\end{tabular}




\begin{tabular}{|c|c|c|c|c|c|c|c|c|c|c|c|c|c|c|}
\hline NJ160 & NJ160 & $2021 / 7 / 26$ & $2021 / 7 / 27$ & II & Moderate & OPS & NJ & multiplex-pcr & BGI & 18 & 22 & $4,165,046$ & $99.88 \%$ & 8368.15 \\
\hline NJ162 & NJ162 & $2021 / 7 / 27$ & $2021 / 7 / 31$ & $\mathrm{CI}$ & Moderate & OPS & NJ & multiplex-pcr & Illumina & 21.2 & 19.4 & 930,888 & $99.73 \%$ & 2839.635 \\
\hline NJ164 & NJ164 & $2021 / 7 / 26$ & $2021 / 8 / 10$ & II & Moderate & OPS & NJ & multiplex-pcr & Illumina & 26 & 27 & 206,891 & $99.45 \%$ & 811.6303 \\
\hline NJ165 & NJ165 & $2021 / 7 / 28$ & $2021 / 7 / 31$ & $\mathrm{CI}$ & Moderate & OPS & NJ & multiplex-pcr & Illumina & 20.3 & 19.3 & 207,893 & $99.31 \%$ & 614.5542 \\
\hline NJ166 & NJ166 & $2021 / 7 / 27$ & $2021 / 7 / 31$ & $\mathrm{CI}$ & Moderate & OPS & NJ & multiplex-pcr & BGI & 36.1 & 36.7 & 170,059 & $99.78 \%$ & 567.0518 \\
\hline NJ167 & NJ167 & $2021 / 7 / 27$ & $2021 / 7 / 31$ & CI & Moderate & OPS & NJ & multiplex-pcr & Illumina & 28.6 & 29.5 & $1,994,734$ & $99.56 \%$ & 7735.701 \\
\hline NJ168 & NJ168 & $2021 / 7 / 27$ & $2021 / 7 / 31$ & $\mathrm{CI}$ & Moderate & OPS & NJ & multiplex-pcr & Illumina & 29.2 & 28 & $1,981,999$ & $99.57 \%$ & 7442.95 \\
\hline NJ175 & NJ175 & $2021 / 7 / 27$ & $2021 / 7 / 31$ & $\mathrm{CI}$ & Mild & OPS & NJ & multiplex-pcr & Illumina & 25.4 & 23.9 & $1,148,512$ & $99.68 \%$ & 3378.314 \\
\hline NJ176 & NJ176 & $2021 / 7 / 27$ & $2021 / 7 / 31$ & CI & Mild & OPS & NJ & multiplex-pcr & Illumina & 29.4 & 27.8 & $1,615,010$ & $99.52 \%$ & 6120.011 \\
\hline NJ177 & NJ177 & $2021 / 7 / 28$ & $2021 / 7 / 31$ & CI & Moderate & OPS & NJ & multiplex-pcr & Illumina & 29.1 & 28.1 & 915,298 & $99.54 \%$ & 3627.126 \\
\hline NJ179 & NJ179 & $2021 / 7 / 28$ & $2021 / 7 / 31$ & CI & Moderate & OPS & NJ & multiplex-pcr & Illumina & 20.4 & 18.3 & $1,348,116$ & $99.78 \%$ & 4041.57 \\
\hline NJ182 & NJ182 & $2021 / 7 / 29$ & $2021 / 7 / 31$ & CI & Moderate & OPS & NJ & multiplex-pcr & Illumina & 27.2 & 24.6 & $2,025,271$ & $99.56 \%$ & 7723.182 \\
\hline NJ184 & NJ184 & $2021 / 7 / 28$ & $2021 / 7 / 31$ & CI & Moderate & OPS & NJ & multiplex-pcr & BGI & 36.4 & 35.2 & 327,835 & $99.89 \%$ & 1093.516 \\
\hline NJ186 & NJ186 & $2021 / 7 / 29$ & $2021 / 8 / 10$ & $\mathrm{CI}$ & Moderate & OPS & NJ & multiplex-pcr & Illumina & 33 & 33 & 24,917 & $98.92 \%$ & 100.0977 \\
\hline NJ187 & NJ187 & $2021 / 7 / 29$ & $2021 / 7 / 31$ & CI & Moderate & OPS & NJ & multiplex-pcr & Illumina & 21.8 & 20.8 & $1,040,421$ & $99.67 \%$ & 3154.95 \\
\hline NJ188 & NJ188 & $2021 / 7 / 28$ & $2021 / 7 / 31$ & CI & Moderate & OPS & NJ & multiplex-pcr & Illumina & 27.9 & 26.5 & $1,326,499$ & $99.55 \%$ & 5174.659 \\
\hline NJ189 & NJ189 & $2021 / 7 / 30$ & $2021 / 7 / 31$ & CI & Mild & OPS & $\mathrm{NJ}$ & multiplex-pcr & Illumina & 27.9 & 26.4 & $1,061,889$ & $99.62 \%$ & 3186.831 \\
\hline NJ192 & $\mathrm{NJ} 192$ & $2021 / 7 / 30$ & $2021 / 7 / 31$ & $\mathrm{CI}$ & Moderate & OPS & NJ & multiplex-pcr & Illumina & 25.4 & 23.9 & $1,508,078$ & $99.60 \%$ & 5824.268 \\
\hline NJ193 & NJ193 & $2021 / 7 / 30$ & $2021 / 8 / 10$ & CI & Mild & OPS & NJ & multiplex-pcr & $\mathrm{TF}$ & 30 & 30 & $2,329,786$ & $99.75 \%$ & 15682.61 \\
\hline NJ195 & NJ195 & $2021 / 7 / 24$ & $2021 / 7 / 31$ & II & Moderate & OPS & NJ & multiplex-pcr & $\mathrm{TF}$ & 39 & 39 & $2,139,455$ & $99.86 \%$ & 14813.98 \\
\hline NJ196 & NJ196 & $2021 / 7 / 28$ & $2021 / 8 / 3$ & CI & Moderate & OPS & NJ & multiplex-pcr & $\mathrm{TF}$ & 32 & 30 & $2,686,673$ & $99.88 \%$ & 15400.34 \\
\hline NJ197 & NJ197 & $2021 / 7 / 30$ & $2021 / 8 / 3$ & $\mathrm{CI}$ & Mild & OPS & NJ & multiplex-pcr & $\mathrm{TF}$ & 25 & 27 & $3,707,905$ & $99.89 \%$ & 25547.63 \\
\hline NJ198 & NJ198 & $2021 / 7 / 30$ & $2021 / 8 / 3$ & CI & Moderate & OPS & NJ & multiplex-pcr & $\mathrm{TF}$ & 26 & 27 & 647,062 & $99.82 \%$ & 4427.391 \\
\hline NJ199 & NJ199 & $2021 / 7 / 31$ & $2021 / 8 / 10$ & CI & Moderate & OPS & NJ & multiplex-pcr & $\mathrm{TF}$ & 30 & 30 & $1,365,684$ & $99.78 \%$ & 9089.513 \\
\hline NJ200 & NJ200 & $2021 / 7 / 31$ & $2021 / 8 / 3$ & $\mathrm{CI}$ & Moderate & OPS & NJ & multiplex-pcr & $\mathrm{TF}$ & 22 & 24 & $5,636,384$ & $99.83 \%$ & 39085.66 \\
\hline NJ201 & NJ201 & $2021 / 7 / 31$ & $2021 / 8 / 3$ & CI & Moderate & OPS & NJ & multiplex-pcr & $\mathrm{TF}$ & 25 & 27 & $2,350,858$ & $99.83 \%$ & 16234.55 \\
\hline NJ203 & NJ203 & $2021 / 7 / 30$ & $2021 / 8 / 10$ & $\mathrm{CI}$ & Moderate & OPS & NJ & multiplex-pcr & $\mathrm{TF}$ & 33 & 33 & $1,372,493$ & $99.78 \%$ & 9263.599 \\
\hline NJ204 & NJ204 & $2021 / 7 / 28$ & $2021 / 8 / 3$ & $\mathrm{CI}$ & Mild & OPS & NJ & multiplex-pcr & $\mathrm{TF}$ & 23 & 25 & $4,055,842$ & $99.85 \%$ & 28033.93 \\
\hline NJ205 & NJ205 & 2021/7/31 & $2021 / 8 / 3$ & CI & Mild & OPS & NJ & multiplex-pcr & $\mathrm{TF}$ & 26 & 28 & $1,019,838$ & $99.79 \%$ & 7040.493 \\
\hline
\end{tabular}




\begin{tabular}{|c|c|c|c|c|c|c|c|c|c|c|c|c|c|c|}
\hline NJ206 & NJ206 & $2021 / 7 / 31$ & $2021 / 8 / 3$ & $\mathrm{CI}$ & Moderate & OPS & $\mathrm{NJ}$ & multiplex-pcr & TF & 18 & 20 & $5,428,442$ & $99.92 \%$ & 29739.42 \\
\hline NJ207 & NJ207 & $2021 / 7 / 31$ & $2021 / 8 / 3$ & $\mathrm{CI}$ & Moderate & OPS & NJ & multiplex-pcr & TF & 14 & 16 & $5,778,873$ & $99.83 \%$ & 37018.06 \\
\hline NJ208 & NJ208 & $2021 / 7 / 22$ & $2021 / 8 / 3$ & II & Mild & OPS & NJ & multiplex-pcr & TF & 32 & 33 & $1,654,095$ & $99.82 \%$ & 10618.64 \\
\hline NJ209 & NJ209 & $2021 / 7 / 30$ & $2021 / 8 / 3$ & $\mathrm{CI}$ & Moderate & OPS & NJ & multiplex-pcr & TF & 24 & 27 & $4,016,786$ & $99.84 \%$ & 25074.3 \\
\hline NJ210 & NJ210 & $2021 / 7 / 30$ & $2021 / 8 / 3$ & $\mathrm{CI}$ & Mild & OPS & NJ & multiplex-pcr & TF & 17 & 20 & $3,074,352$ & $99.78 \%$ & 17238.55 \\
\hline NJ211 & NJ211 & $2021 / 7 / 29$ & $2021 / 8 / 3$ & $\mathrm{CI}$ & Moderate & OPS & NJ & multiplex-pcr & TF & 23 & 24 & $3,905,947$ & $99.82 \%$ & 26785.25 \\
\hline NJ212 & NJ212 & $2021 / 7 / 31$ & $2021 / 8 / 3$ & $\mathrm{CI}$ & Mild & OPS & NJ & multiplex-pcr & TF & 17 & 15 & $3,038,581$ & $99.83 \%$ & 15513.77 \\
\hline NJ213 & NJ213 & $2021 / 7 / 31$ & $2021 / 8 / 3$ & $\mathrm{CI}$ & Mild & OPS & $\mathrm{NJ}$ & multiplex-pcr & TF & 37 & 36 & $1,195,453$ & $99.56 \%$ & 5174.975 \\
\hline NJ215 & NJ215 & $2021 / 7 / 31$ & $2021 / 8 / 3$ & $\mathrm{CI}$ & Moderate & OPS & NJ & multiplex-pcr & $\mathrm{TF}$ & 31 & 33 & $2,874,080$ & $99.87 \%$ & 18781.26 \\
\hline NJ217 & NJ217 & $2021 / 7 / 31$ & $2021 / 8 / 3$ & $\mathrm{CI}$ & Moderate & OPS & NJ & multiplex-pcr & TF & 13 & 15 & $4,053,568$ & $99.92 \%$ & 24870.71 \\
\hline NJ218 & NJ218 & $2021 / 8 / 1$ & $2021 / 8 / 10$ & $\mathrm{CI}$ & Moderate & OPS & NJ & multiplex-pcr & TF & 28 & 29 & $2,451,953$ & $99.79 \%$ & 16057.24 \\
\hline NJ220 & NJ220 & $2021 / 7 / 31$ & $2021 / 8 / 3$ & $\mathrm{CI}$ & Moderate & OPS & NJ & multiplex-pcr & TF & 15 & 19 & $4,713,137$ & $99.84 \%$ & 30434.29 \\
\hline NJ221 & NJ221 & $2021 / 8 / 2$ & $2021 / 8 / 10$ & $\mathrm{CI}$ & Moderate & OPS & NJ & multiplex-pcr & TF & 30 & 30 & $1,979,837$ & $99.78 \%$ & 13042.76 \\
\hline NJ222 & NJ222 & $2021 / 7 / 30$ & 2021/8/6 & $\mathrm{CI}$ & Moderate & OPS & NJ & multiplex-pcr & TF & 18 & 20 & $2,912,071$ & $99.67 \%$ & 16475.41 \\
\hline NJ223 & NJ223 & $2021 / 7 / 26$ & $2021 / 8 / 6$ & $\mathrm{CI}$ & Mild & NPS & $\mathrm{NJ}$ & multiplex-pcr & Illumina & 30 & 30 & 335,666 & $98.79 \%$ & 1037.277 \\
\hline NJ224 & NJ224 & $2021 / 8 / 2$ & $2021 / 8 / 6$ & $\mathrm{CI}$ & Moderate & NPS & $\mathrm{NJ}$ & multiplex-pcr & Illumina & 16 & 19 & 736,787 & $99.59 \%$ & 2887.592 \\
\hline NJ226 & NJ226 & 2021/8/1 & $2021 / 8 / 4$ & $\mathrm{CI}$ & Moderate & OPS & NJ & multiplex-pcr & Illumina & 35 & 33 & $2,021,475$ & $99.76 \%$ & 7442.81 \\
\hline NJ227 & NJ227 & $2021 / 8 / 3$ & $2021 / 8 / 4$ & $\mathrm{CI}$ & Moderate & OPS & NJ & multiplex-pcr & Illumina & 34 & 33 & $1,193,633$ & $99.70 \%$ & 4344.945 \\
\hline NJ228 & NJ228 & $2021 / 8 / 4$ & $2021 / 8 / 6$ & CI & Moderate & OPS & NJ & multiplex-pcr & Illumina & 38 & 36 & 862,254 & $99.87 \%$ & 3436.348 \\
\hline NJ229 & NJ229 & $2021 / 8 / 5$ & 2021/8/6 & ER & Moderate & NPS & NJ & multiplex-pcr & Illumina & 36 & 36 & 670,441 & $99.41 \%$ & 2329.684 \\
\hline NJ230 & NJ230 & 2021/8/6 & $2021 / 8 / 7$ & ER & Moderate & NPS & $\mathrm{NJ}$ & multiplex-pcr & Illumina & 19 & 18 & 544,726 & $99.82 \%$ & 1882.677 \\
\hline NJ232 & NJ232 & $2021 / 8 / 8$ & 2021/8/9 & ER & Moderate & OPS & NJ & multiplex-pcr & Illumina & 24 & 25 & $23,233,671$ & $99.93 \%$ & 90935.46 \\
\hline $\mathrm{NJ} 234$ & NJ234 & $2021 / 8 / 10$ & $2021 / 8 / 14$ & ER & Moderate & OPS & NJ & multiplex-pcr & Illumina & 20 & 21 & $1,643,699$ & $99.94 \%$ & 6532.425 \\
\hline NJ235 & NJ235 & $2021 / 8 / 11$ & $2021 / 8 / 13$ & ER & Moderate & OPS & NJ & multiplex-pcr & BGI & 28 & 26 & $12,826,523$ & $99.93 \%$ & 40712.12 \\
\hline YZ001 & YZ001 & $2021 / 7 / 23$ & $2021 / 7 / 28$ & $\mathrm{NE}$ & Moderate & OPS & YZ & multiplex-pcr & Illumina & 19 & 20 & $2,712,182$ & $99.92 \%$ & 7319.275 \\
\hline YZ002 & YZ002 & $2021 / 7 / 25$ & $2021 / 8 / 2$ & NE & Moderate & OPS & YZ & multiplex-pcr & Illumina & 16 & 16 & $58,468,029$ & $99.93 \%$ & 165219.4 \\
\hline YZ003 & YZ003 & $2021 / 7 / 25$ & $2021 / 8 / 2$ & $\mathrm{NE}$ & Moderate & OPS & YZ & multiplex-pcr & Illumina & 18 & 18 & $36,241,018$ & $99.92 \%$ & 100101.2 \\
\hline YZ004 & YZ004 & $2021 / 7 / 27$ & $2021 / 8 / 2$ & $\mathrm{NE}$ & Moderate & OPS & YZ & multiplex-pcr & Illumina & 18 & 18 & $8,596,632$ & $99.92 \%$ & 17094.83 \\
\hline YZ005 & YZ005 & $2021 / 7 / 28$ & $2021 / 8 / 2$ & $\mathrm{NE}$ & Moderate & OPS & YZ & multiplex-pcr & Illumina & 18 & 18 & $32,763,885$ & $99.94 \%$ & 90837.97 \\
\hline
\end{tabular}




\begin{tabular}{|c|c|c|c|c|c|c|c|c|c|c|c|c|c|c|}
\hline YZ006 & YZ006 & $2021 / 7 / 25$ & $2021 / 8 / 2$ & $\mathrm{NE}$ & Moderate & OPS & $\mathrm{YZ}$ & multiplex-pcr & BGI & 18 & 19 & $9,629,323$ & $99.87 \%$ & 23400.49 \\
\hline YZ007 & YZ007 & $2021 / 7 / 28$ & $2021 / 8 / 2$ & $\mathrm{NE}$ & Moderate & OPS & YZ & multiplex-pcr & Illumina & 30 & 30 & $52,444,838$ & $99.93 \%$ & 137320 \\
\hline YZ009 & YZ009 & $2021 / 7 / 25$ & $2021 / 8 / 2$ & $\mathrm{NE}$ & Moderate & OPS & YZ & multiplex-pcr & Illumina & 18 & 14 & $69,834,847$ & $99.92 \%$ & 177166.5 \\
\hline YZ010 & YZ010 & $2021 / 7 / 27$ & $2021 / 8 / 10$ & $\mathrm{NE}$ & Severe & OPS & YZ & multiplex-pcr & Illumina & 26 & 26 & $1,347,834$ & $99.30 \%$ & 5408.447 \\
\hline YZ012 & YZ012 & $2021 / 7 / 26$ & $2021 / 8 / 2$ & $\mathrm{NE}$ & Moderate & OPS & YZ & multiplex-pcr & Illumina & 20 & 18 & $44,594,971$ & $99.92 \%$ & 113449 \\
\hline YZ013 & YZ013 & $2021 / 7 / 29$ & $2021 / 8 / 2$ & $\mathrm{NE}$ & Moderate & OPS & YZ & multiplex-pcr & Illumina & 18 & 18 & $57,395,337$ & $99.92 \%$ & 153102.3 \\
\hline YZ014 & YZ014 & $2021 / 7 / 29$ & $2021 / 8 / 2$ & $\mathrm{NE}$ & Critical & OPS & YZ & multiplex-pcr & Illumina & 36 & 34 & $64,707,878$ & $99.92 \%$ & 176122.7 \\
\hline YZ015 & YZ015 & $2021 / 7 / 28$ & $2021 / 8 / 2$ & $\mathrm{NE}$ & Critical & OPS & YZ & multiplex-pcr & Illumina & 28 & 28 & $26,392,447$ & $99.92 \%$ & 54900.49 \\
\hline YZ016 & YZ016 & $2021 / 7 / 28$ & $2021 / 8 / 2$ & $\mathrm{NE}$ & Moderate & OPS & YZ & multiplex-pcr & Illumina & 17 & 17 & $2,917,680$ & $99.94 \%$ & 10812.31 \\
\hline YZ018 & YZ018 & $2021 / 7 / 29$ & $2021 / 8 / 2$ & $\mathrm{NE}$ & Moderate & NPS & YZ & multiplex-pcr & Illumina & 16 & 16 & $15,010,240$ & $99.87 \%$ & 55382.95 \\
\hline YZ019 & YZ019 & $2021 / 7 / 29$ & $2021 / 8 / 4$ & $\mathrm{NE}$ & Severe & OPS & YZ & multiplex-pcr & $\mathrm{TF}$ & 30 & 31 & $1,779,230$ & $99.75 \%$ & 12514.63 \\
\hline YZ020 & YZ020 & $2021 / 7 / 28$ & $2021 / 8 / 2$ & $\mathrm{NE}$ & Moderate & OPS & YZ & multiplex-pcr & Illumina & 18 & 18 & $2,227,180$ & $99.94 \%$ & 8663.303 \\
\hline YZ021 & YZ021 & $2021 / 7 / 24$ & $2021 / 8 / 2$ & $\mathrm{NE}$ & Moderate & OPS & YZ & multiplex-pcr & Illumina & 30 & 30 & $16,018,498$ & $99.94 \%$ & 62475.28 \\
\hline YZ023 & YZ023 & $2021 / 7 / 26$ & $2021 / 8 / 2$ & $\mathrm{NE}$ & Moderate & OPS & YZ & multiplex-pcr & Illumina & 14 & 12 & $13,963,501$ & $99.94 \%$ & 54454.75 \\
\hline YZ025 & YZ025 & $2021 / 7 / 29$ & $2021 / 8 / 2$ & $\mathrm{NE}$ & Mild & OPS & YZ & multiplex-pcr & Illumina & 26 & 26 & $2,812,766$ & $99.93 \%$ & 10640.39 \\
\hline YZ026 & YZ026 & $2021 / 7 / 24$ & $2021 / 8 / 2$ & $\mathrm{NE}$ & Moderate & OPS & YZ & multiplex-pcr & Illumina & 28 & 26 & $2,584,161$ & $99.94 \%$ & 9893.152 \\
\hline YZ029 & YZ029 & $2021 / 7 / 29$ & $2021 / 8 / 2$ & $\mathrm{NE}$ & Moderate & OPS & YZ & multiplex-pcr & Illumina & 16 & 16 & $21,371,244$ & $99.94 \%$ & 82914.15 \\
\hline YZ030 & YZ030 & $2021 / 7 / 30$ & $2021 / 8 / 2$ & II & Moderate & OPS & YZ & multiplex-pcr & BGI & 30 & 30 & 611,093 & $99.77 \%$ & 1479.57 \\
\hline YZ031 & YZ031 & $2021 / 7 / 30$ & $2021 / 8 / 2$ & II & Critical & OPS & YZ & multiplex-pcr & BGI & 28 & 27 & $7,674,117$ & $99.87 \%$ & 18306.22 \\
\hline YZ032 & YZ032 & $2021 / 7 / 31$ & $2021 / 8 / 2$ & II & Moderate & OPS & YZ & multiplex-pcr & BGI & 30 & 28 & $1,875,242$ & $99.82 \%$ & 4569.217 \\
\hline YZ033 & YZ033 & $2021 / 7 / 30$ & $2021 / 8 / 4$ & $\mathrm{NE}$ & Moderate & OPS & YZ & multiplex-pcr & $\mathrm{TF}$ & 30 & 29 & $3,253,177$ & $99.81 \%$ & 19820.28 \\
\hline YZ034 & YZ034 & $2021 / 7 / 30$ & $2021 / 8 / 2$ & $\mathrm{NE}$ & Moderate & OPS & YZ & multiplex-pcr & BGI & 24 & 25 & $9,561,798$ & $99.87 \%$ & 23011.31 \\
\hline YZ035 & YZ035 & $2021 / 7 / 28$ & $2021 / 8 / 2$ & $\mathrm{NE}$ & Critical & OPS & YZ & multiplex-pcr & BGI & 33 & 33 & $4,420,638$ & $99.76 \%$ & 10363.18 \\
\hline YZ036 & YZ036 & $2021 / 7 / 29$ & $2021 / 8 / 2$ & $\mathrm{NE}$ & Moderate & OPS & YZ & multiplex-pcr & BGI & 11 & 13 & $3,511,111$ & $99.87 \%$ & 8343.174 \\
\hline YZ037 & YZ037 & $2021 / 7 / 30$ & $2021 / 8 / 2$ & II & Moderate & OPS & YZ & multiplex-pcr & Illumina & 24 & 24 & $21,821,627$ & $99.94 \%$ & 85312.91 \\
\hline YZ038 & YZ038 & $2021 / 7 / 28$ & $2021 / 8 / 2$ & $\mathrm{NE}$ & Moderate & OPS & YZ & multiplex-pcr & Illumina & 20 & 20 & $2,683,209$ & $99.94 \%$ & 10163.03 \\
\hline YZ039 & YZ039 & $2021 / 7 / 31$ & $2021 / 8 / 2$ & II & Moderate & OPS & YZ & multiplex-pcr & Illumina & 36 & 32 & $17,285,078$ & $99.94 \%$ & 66818.34 \\
\hline YZ041 & YZ041 & $2021 / 7 / 30$ & $2021 / 8 / 2$ & $\mathrm{NE}$ & Moderate & OPS & YZ & multiplex-pcr & Illumina & 16 & 14 & $9,218,644$ & $99.90 \%$ & 35912.87 \\
\hline YZ043 & YZ043 & $2021 / 7 / 29$ & $2021 / 8 / 2$ & $\mathrm{NE}$ & Moderate & OPS & YZ & multiplex-pcr & Illumina & 20 & 20 & $10,396,251$ & $99.93 \%$ & 39480.57 \\
\hline
\end{tabular}




\begin{tabular}{|c|c|c|c|c|c|c|c|c|c|c|c|c|c|c|}
\hline YZ044 & YZ044 & $2021 / 7 / 28$ & $2021 / 8 / 2$ & $\mathrm{NE}$ & Moderate & OPS & YZ & multiplex-pcr & Illumina & 22 & 22 & $15,465,439$ & $99.94 \%$ & 60372.59 \\
\hline YZ045 & YZ045 & $2021 / 7 / 28$ & $2021 / 8 / 2$ & $\mathrm{NE}$ & Critical & OPS & YZ & multiplex-pcr & Illumina & 22 & 22 & $11,045,899$ & $99.93 \%$ & 42928.45 \\
\hline YZ046 & YZ046 & $2021 / 7 / 29$ & $2021 / 8 / 2$ & $\mathrm{NE}$ & Moderate & OPS & YZ & multiplex-pcr & Illumina & 26 & 26 & $8,661,265$ & $99.94 \%$ & 34165.79 \\
\hline YZ047 & YZ047 & $2021 / 7 / 30$ & $2021 / 8 / 2$ & II & Moderate & OPS & YZ & multiplex-pcr & Illumina & 26 & 26 & $8,709,930$ & $99.94 \%$ & 33456.66 \\
\hline YZ048 & YZ048 & $2021 / 7 / 29$ & $2021 / 8 / 2$ & $\mathrm{NE}$ & Mild & OPS & YZ & multiplex-pcr & BGI & 35 & 34 & 128,185 & $99.75 \%$ & 305.8903 \\
\hline YZ049 & YZ049 & $2021 / 7 / 26$ & $2021 / 8 / 2$ & $\mathrm{NE}$ & Moderate & OPS & YZ & multiplex-pcr & Illumina & 18 & 16 & $9,749,776$ & $99.94 \%$ & 37718.75 \\
\hline YZ050 & YZ050 & $2021 / 7 / 28$ & $2021 / 8 / 2$ & $\mathrm{NE}$ & Moderate & OPS & YZ & multiplex-pcr & Illumina & 30 & 28 & $10,313,725$ & $99.94 \%$ & 40392.38 \\
\hline YZ051 & YZ051 & $2021 / 7 / 25$ & $2021 / 8 / 2$ & $\mathrm{NE}$ & Moderate & OPS & YZ & multiplex-pcr & BGI & 15 & 16 & $8,365,427$ & $99.87 \%$ & 19913.8 \\
\hline YZ052 & YZ052 & $2021 / 7 / 30$ & $2021 / 8 / 2$ & $\mathrm{NE}$ & Moderate & OPS & YZ & multiplex-pcr & Illumina & 30 & 24 & $15,001,500$ & $99.94 \%$ & 57833.11 \\
\hline YZ053 & YZ053 & $2021 / 7 / 29$ & $2021 / 8 / 2$ & $\mathrm{NE}$ & Moderate & OPS & YZ & multiplex-pcr & Illumina & 26 & 24 & $12,749,496$ & $99.94 \%$ & 49413.07 \\
\hline YZ054 & YZ054 & $2021 / 7 / 30$ & $2021 / 8 / 2$ & $\mathrm{NE}$ & Moderate & OPS & YZ & multiplex-pcr & Illumina & 32 & 32 & $10,703,647$ & $99.94 \%$ & 42188.11 \\
\hline YZ056 & YZ056 & $2021 / 7 / 31$ & $2021 / 8 / 4$ & II & Moderate & OPS & YZ & multiplex-pcr & TF & 29 & 29.6 & $1,760,781$ & $99.81 \%$ & 12293.7 \\
\hline YZ057 & YZ057 & $2021 / 7 / 31$ & $2021 / 8 / 2$ & II & Critical & OPS & YZ & multiplex-pcr & BGI & 33 & 33 & $2,299,706$ & $99.82 \%$ & 5468.761 \\
\hline YZ058 & YZ058 & $2021 / 7 / 31$ & $2021 / 8 / 2$ & II & Severe & OPS & YZ & multiplex-pcr & TF & 10 & 9 & $6,754,215$ & $99.94 \%$ & 42702.32 \\
\hline YZ059 & YZ059 & $2021 / 7 / 29$ & $2021 / 8 / 2$ & II & Moderate & OPS & YZ & multiplex-pcr & $\mathrm{TF}$ & 30.8 & 29.5 & 366,294 & $99.82 \%$ & 2137.946 \\
\hline YZ060 & YZ060 & $2021 / 7 / 28$ & $2021 / 8 / 10$ & $\mathrm{NE}$ & Moderate & OPS & YZ & multiplex-pcr & TF & 26 & 26 & $1,989,538$ & $99.81 \%$ & 12870.68 \\
\hline YZ061 & YZ061 & $2021 / 7 / 31$ & $2021 / 8 / 4$ & II & Moderate & OPS & YZ & multiplex-pcr & Illumina & 24 & 25 & 382,249 & $99.61 \%$ & 1520.48 \\
\hline YZ062 & YZ062 & $2021 / 7 / 31$ & $2021 / 8 / 4$ & II & Moderate & OPS & YZ & multiplex-pcr & TF & 16 & 14 & $5,822,047$ & $99.84 \%$ & 37460.54 \\
\hline YZ063 & YZ063 & $2021 / 7 / 31$ & $2021 / 8 / 2$ & II & Moderate & OPS & YZ & multiplex-pcr & BGI & 18 & 20 & $8,266,380$ & $99.86 \%$ & 19679.56 \\
\hline YZ064 & YZ064 & $2021 / 7 / 31$ & $2021 / 8 / 2$ & II & Mild & OPS & YZ & multiplex-pcr & BGI & 12 & 15 & $1,059,825$ & $99.47 \%$ & 2518.665 \\
\hline YZ065 & YZ065 & $2021 / 8 / 1$ & $2021 / 8 / 4$ & II & Moderate & OPS & YZ & multiplex-pcr & $\mathrm{TF}$ & 37 & 30 & 100,167 & $99.56 \%$ & 701.6162 \\
\hline YZ066 & YZ066 & 2021/8/1 & $2021 / 8 / 2$ & II & Mild & OPS & YZ & multiplex-pcr & BGI & 25 & 25 & $9,546,646$ & $99.87 \%$ & 23531.25 \\
\hline YZ067 & YZ067 & $2021 / 7 / 31$ & $2021 / 8 / 2$ & II & Moderate & OPS & YZ & multiplex-pcr & BGI & 14 & 18 & $8,243,515$ & $99.94 \%$ & 19534.97 \\
\hline YZ069 & YZ069 & $2021 / 7 / 31$ & $2021 / 8 / 2$ & II & Moderate & OPS & YZ & multiplex-pcr & BGI & 22 & 23 & $8,357,344$ & $99.86 \%$ & 20053.69 \\
\hline YZ070 & YZ070 & $2021 / 7 / 28$ & $2021 / 8 / 2$ & $\mathrm{NE}$ & Moderate & OPS & YZ & multiplex-pcr & Illumina & 20 & 20 & $2,417,562$ & $99.94 \%$ & 9297.844 \\
\hline YZ074 & YZ074 & $2021 / 7 / 29$ & $2021 / 8 / 2$ & $\mathrm{NE}$ & Moderate & OPS & $\mathrm{YZ}$ & multiplex-pcr & BGI & 32 & 31 & $1,327,834$ & $99.75 \%$ & 3175.667 \\
\hline YZ076 & YZ076 & $2021 / 7 / 30$ & $2021 / 8 / 2$ & II & Moderate & OPS & YZ & multiplex-pcr & BGI & 20 & 21 & $11,193,272$ & $99.86 \%$ & 26883.59 \\
\hline YZ077 & YZ077 & $2021 / 7 / 30$ & $2021 / 8 / 4$ & II & Moderate & OPS & YZ & multiplex-pcr & $\mathrm{TF}$ & 23 & 23.5 & 941,026 & $99.79 \%$ & 5562.444 \\
\hline YZ079 & YZ079 & $2021 / 7 / 31$ & $2021 / 8 / 4$ & II & Moderate & OPS & YZ & multiplex-pcr & TF & 24.5 & 25 & 713,348 & $99.72 \%$ & 4244.41 \\
\hline
\end{tabular}




\begin{tabular}{|c|c|c|c|c|c|c|c|c|c|c|c|c|c|c|}
\hline YZ080 & YZ080 & $2021 / 7 / 24$ & $2021 / 8 / 2$ & $\mathrm{NE}$ & Moderate & OPS & $\mathrm{YZ}$ & multiplex-pcr & BGI & 17 & 19 & $18,803,063$ & $99.87 \%$ & 45081.26 \\
\hline YZ081 & YZ081 & $2021 / 7 / 26$ & $2021 / 8 / 2$ & $\mathrm{NE}$ & Critical & OPS & YZ & multiplex-pcr & BGI & 12 & 14 & $7,317,739$ & $99.94 \%$ & 17501.15 \\
\hline YZ082 & YZ082 & $2021 / 7 / 28$ & $2021 / 8 / 2$ & $\mathrm{NE}$ & Severe & OPS & YZ & multiplex-pcr & Illumina & 37 & 27 & $5,224,634$ & $99.94 \%$ & 20784.64 \\
\hline YZ083 & YZ083 & $2021 / 7 / 29$ & $2021 / 8 / 4$ & II & Moderate & OPS & YZ & multiplex-pcr & $\mathrm{TF}$ & 28 & 27 & $2,795,146$ & $99.83 \%$ & 19406.64 \\
\hline YZ084 & YZ084 & $2021 / 7 / 31$ & $2021 / 8 / 4$ & II & Moderate & OPS & YZ & multiplex-pcr & $\mathrm{TF}$ & 14 & 14 & $3,967,031$ & $99.89 \%$ & 25247.41 \\
\hline YZ085 & YZ085 & $2021 / 8 / 1$ & 2021/8/10 & II & Moderate & OPS & YZ & multiplex-pcr & Illumina & 25 & 26 & 126,134 & $99.51 \%$ & 509.782 \\
\hline YZ086 & YZ086 & $2021 / 8 / 1$ & $2021 / 8 / 4$ & II & Moderate & OPS & YZ & multiplex-pcr & $\mathrm{TF}$ & 29.8 & 29.1 & $1,669,001$ & $99.73 \%$ & 11025.46 \\
\hline YZ087 & YZ087 & $2021 / 7 / 29$ & $2021 / 8 / 2$ & $\mathrm{NE}$ & Moderate & OPS & YZ & multiplex-pcr & BGI & 11 & 14 & $3,896,582$ & $99.82 \%$ & 9265.278 \\
\hline YZ089 & YZ089 & $2021 / 7 / 30$ & $2021 / 8 / 4$ & $\mathrm{NE}$ & Moderate & OPS & YZ & multiplex-pcr & $\mathrm{TF}$ & 11.6 & 15.6 & $1,036,643$ & $99.78 \%$ & 6065.386 \\
\hline YZ091 & YZ091 & $2021 / 8 / 1$ & $2021 / 8 / 18$ & II & Critical & OPS & YZ & multiplex-pcr & Illumina & 33 & 29 & $2,361,315$ & $99.86 \%$ & 9001.676 \\
\hline YZ092 & YZ092 & $2021 / 7 / 31$ & $2021 / 8 / 4$ & II & Mild & OPS & YZ & multiplex-pcr & $\mathrm{TF}$ & 24 & 24 & $2,527,051$ & $99.77 \%$ & 17389.03 \\
\hline YZ094 & YZ094 & $2021 / 8 / 1$ & $2021 / 8 / 4$ & II & Moderate & OPS & YZ & multiplex-pcr & $\mathrm{TF}$ & 22.8 & 25 & $5,262,546$ & $99.77 \%$ & 36825.15 \\
\hline YZ095 & YZ095 & $2021 / 7 / 27$ & $2021 / 8 / 2$ & $\mathrm{NE}$ & Moderate & OPS & YZ & multiplex-pcr & BGI & 15 & 15 & $8,457,881$ & $99.86 \%$ & 20202.7 \\
\hline YZ096 & YZ096 & $2021 / 7 / 31$ & $2021 / 8 / 4$ & II & Moderate & OPS & YZ & multiplex-pcr & $\mathrm{TF}$ & 28 & 28 & $2,439,837$ & $99.68 \%$ & 14895.12 \\
\hline YZ097 & YZ097 & $2021 / 7 / 31$ & $2021 / 8 / 2$ & II & Mild & OPS & YZ & multiplex-pcr & BGI & 31 & 32 & 868,847 & $99.79 \%$ & 2075.863 \\
\hline YZ098 & YZ098 & $2021 / 7 / 31$ & $2021 / 8 / 2$ & II & Moderate & OPS & YZ & multiplex-pcr & BGI & 32 & 30 & 533,844 & $99.76 \%$ & 1278.872 \\
\hline YZ099 & YZ099 & $2021 / 8 / 1$ & $2021 / 8 / 4$ & $\mathrm{NE}$ & Moderate & OPS & YZ & multiplex-pcr & $\mathrm{TF}$ & 26 & 26 & $3,620,921$ & $99.77 \%$ & 23267.64 \\
\hline YZ100 & YZ100 & $2021 / 8 / 1$ & $2021 / 8 / 4$ & II & Severe & OPS & YZ & multiplex-pcr & $\mathrm{TF}$ & 21.5 & 21 & $4,584,156$ & $99.83 \%$ & 26154.21 \\
\hline YZ102 & YZ102 & $2021 / 7 / 25$ & $2021 / 8 / 2$ & $\mathrm{NE}$ & Severe & OPS & YZ & multiplex-pcr & Illumina & 14 & 15 & $7,330,743$ & $99.87 \%$ & 17581.64 \\
\hline YZ103 & YZ103 & $2021 / 7 / 31$ & $2021 / 8 / 2$ & II & Severe & OPS & YZ & multiplex-pcr & Illumina & 11 & 13 & $4,579,104$ & $99.85 \%$ & 10873.22 \\
\hline YZ104 & YZ104 & $2021 / 7 / 31$ & $2021 / 8 / 2$ & II & Moderate & OPS & YZ & multiplex-pcr & Illumina & 24 & 24 & $8,290,978$ & $99.87 \%$ & 19951.34 \\
\hline YZ105 & YZ105 & $2021 / 7 / 28$ & $2021 / 8 / 4$ & $\mathrm{NE}$ & Critical & OPS & YZ & multiplex-pcr & Illumina & 21.7 & 21.9 & $2,408,982$ & $99.94 \%$ & 9570.654 \\
\hline YZ106 & YZ106 & $2021 / 8 / 2$ & $2021 / 8 / 4$ & II & Moderate & OPS & YZ & multiplex-pcr & $\mathrm{TF}$ & 28.7 & 29 & 534,516 & $99.56 \%$ & 3070.922 \\
\hline YZ108 & YZ108 & $2021 / 8 / 1$ & $2021 / 8 / 2$ & II & Moderate & OPS & YZ & multiplex-pcr & BGI & 20 & 20 & $10,261,348$ & $99.89 \%$ & 24982.77 \\
\hline YZ109 & YZ109 & $2021 / 7 / 31$ & $2021 / 8 / 4$ & II & Moderate & OPS & YZ & multiplex-pcr & $\mathrm{TF}$ & 11 & 11 & $5,216,616$ & $99.92 \%$ & 32975.43 \\
\hline YZ110 & YZ110 & $2021 / 7 / 30$ & $2021 / 8 / 2$ & $\mathrm{NE}$ & Moderate & OPS & YZ & multiplex-pcr & BGI & 14 & 15 & $13,716,800$ & $99.93 \%$ & 32766.8 \\
\hline YZ112 & YZ112 & $2021 / 7 / 31$ & $2021 / 8 / 2$ & II & Moderate & OPS & YZ & multiplex-pcr & BGI & 22 & 23 & $15,651,848$ & $99.87 \%$ & 38105.87 \\
\hline YZ115 & YZ115 & $2021 / 7 / 31$ & $2021 / 8 / 10$ & II & Moderate & OPS & YZ & multiplex-pcr & Illumina & 25 & 25 & 326,364 & $99.58 \%$ & 1282.524 \\
\hline YZ118 & YZ118 & $2021 / 8 / 2$ & $2021 / 8 / 4$ & II & Mild & OPS & YZ & multiplex-pcr & $\mathrm{TF}$ & 32.5 & 29 & 883,515 & $98.34 \%$ & 4288.538 \\
\hline
\end{tabular}




\begin{tabular}{|c|c|c|c|c|c|c|c|c|c|c|c|c|c|c|}
\hline YZ121 & YZ121 & $2021 / 8 / 1$ & $2021 / 8 / 4$ & II & Mild & OPS & YZ & multiplex-pcr & Illumina & 27.8 & 27.8 & 677,644 & $99.84 \%$ & 2645.484 \\
\hline YZ124 & YZ124 & 2021/8/1 & $2021 / 8 / 4$ & II & Moderate & OPS & YZ & multiplex-pcr & TF & 32.7 & 29 & $1,820,038$ & $99.38 \%$ & 7518.475 \\
\hline YZ125 & YZ125 & $2021 / 7 / 29$ & $2021 / 8 / 2$ & $\mathrm{NE}$ & Mild & OPS & YZ & multiplex-pcr & Illumina & 24 & 24 & $2,693,751$ & $99.94 \%$ & 10449.46 \\
\hline YZ126 & YZ126 & $2021 / 7 / 30$ & $2021 / 8 / 2$ & II & Moderate & OPS & YZ & multiplex-pcr & BGI & 29 & 28 & 534,362 & $99.76 \%$ & 1323.141 \\
\hline YZ128 & YZ128 & $2021 / 8 / 2$ & $2021 / 8 / 10$ & II & Mild & OPS & YZ & multiplex-pcr & TF & 23 & 24 & $2,725,696$ & $99.77 \%$ & 18569.52 \\
\hline YZ129 & YZ129 & $2021 / 8 / 2$ & $2021 / 8 / 10$ & II & Mild & OPS & YZ & multiplex-pcr & Illumina & 20 & 20 & $4,735,926$ & $99.94 \%$ & 18633.52 \\
\hline YZ131 & YZ131 & $2021 / 8 / 2$ & $2021 / 8 / 19$ & II & Mild & OPS & YZ & multiplex-pcr & Illumina & 15 & 15 & $11,921,156$ & $99.94 \%$ & 47331.99 \\
\hline YZ134 & YZ134 & $2021 / 8 / 2$ & $2021 / 8 / 4$ & II & Moderate & OPS & YZ & multiplex-pcr & $\mathrm{TF}$ & 25.7 & 25.4 & $2,613,281$ & $99.82 \%$ & 11572.27 \\
\hline YZ135 & YZ135 & $2021 / 8 / 3$ & $2021 / 8 / 4$ & II & Moderate & OPS & YZ & multiplex-pcr & $\mathrm{TF}$ & 25.4 & 24.2 & $2,113,210$ & $99.84 \%$ & 11769.19 \\
\hline YZ137 & YZ137 & $2021 / 8 / 3$ & $2021 / 8 / 4$ & II & Moderate & OPS & YZ & multiplex-pcr & TF & 24 & 23.4 & $3,481,957$ & $99.83 \%$ & 21265.62 \\
\hline YZ140 & YZ140 & $2021 / 8 / 2$ & $2021 / 8 / 10$ & II & Critical & OPS & YZ & multiplex-pcr & Illumina & 27 & 27 & 291,450 & $99.58 \%$ & 1163.023 \\
\hline YZ143 & YZ143 & $2021 / 7 / 31$ & $2021 / 8 / 10$ & II & Critical & OPS & YZ & multiplex-pcr & Illumina & 30 & 30 & 355,622 & $98.97 \%$ & 1424.866 \\
\hline YZ146 & YZ146 & $2021 / 8 / 2$ & $2021 / 8 / 10$ & II & Moderate & OPS & YZ & multiplex-pcr & Illumina & 29 & 30 & 342,842 & $99.62 \%$ & 1364.552 \\
\hline YZ149 & YZ149 & 2021/8/1 & $2021 / 8 / 2$ & II & Moderate & OPS & YZ & multiplex-pcr & BGI & 13 & 12 & $3,476,175$ & $99.85 \%$ & 8265.455 \\
\hline YZ155 & YZ155 & $2021 / 8 / 4$ & $2021 / 8 / 18$ & II & Moderate & OPS & YZ & multiplex-pcr & Illumina & 30 & 30 & $2,870,743$ & $99.59 \%$ & 10956.42 \\
\hline YZ156 & YZ156 & $2021 / 8 / 3$ & $2021 / 8 / 4$ & II & Moderate & OPS & YZ & multiplex-pcr & $\mathrm{TF}$ & 28.5 & 27 & $4,516,139$ & $99.78 \%$ & 25690.52 \\
\hline YZ159 & YZ159 & $2021 / 8 / 3$ & $2021 / 8 / 11$ & II & Moderate & NPS & YZ & multiplex-pcr & Illumina & 37 & 37 & $2,132,592$ & $99.87 \%$ & 7965.675 \\
\hline YZ160 & YZ160 & $2021 / 8 / 3$ & $2021 / 8 / 11$ & II & Moderate & NPS & YZ & multiplex-pcr & BGI & 35 & 35 & 47,442 & $99.15 \%$ & 156.0289 \\
\hline YZ161 & YZ161 & $2021 / 8 / 2$ & 2021/8/11 & II & Moderate & NPS & YZ & multiplex-pcr & Illumina & 25 & 28 & $2,338,350$ & $99.88 \%$ & 9279.92 \\
\hline YZ162 & YZ162 & $2021 / 8 / 3$ & $2021 / 8 / 11$ & II & Moderate & OPS & YZ & multiplex-pcr & Illumina & 23 & 24 & $14,624,517$ & $99.93 \%$ & 56873.11 \\
\hline YZ163 & YZ163 & 2021/8/1 & $2021 / 8 / 2$ & II & Moderate & OPS & YZ & multiplex-pcr & BGI & 26 & 24 & $19,885,750$ & $99.87 \%$ & 49195.98 \\
\hline YZ168 & YZ168 & $2021 / 7 / 30$ & 2021/8/6 & II & Mild & OPS & YZ & multiplex-pcr & Illumina & 30 & 31 & $1,766,729$ & $99.78 \%$ & 7074.892 \\
\hline YZ169 & YZ169 & $2021 / 8 / 4$ & $2021 / 8 / 6$ & II & Moderate & OPS & YZ & multiplex-pcr & Illumina & 31 & 33 & 711,115 & $99.70 \%$ & 2651.302 \\
\hline YZ170 & YZ170 & $2021 / 7 / 30$ & $2021 / 8 / 11$ & II & Moderate & NPS & YZ & multiplex-pcr & Illumina & 27 & 29 & $1,816,118$ & $99.91 \%$ & 7103.912 \\
\hline YZ171 & YZ171 & $2021 / 8 / 4$ & 2021/8/6 & II & Moderate & OPS & YZ & multiplex-pcr & Illumina & 37 & 35 & $13,927,942$ & $99.89 \%$ & 52769.75 \\
\hline YZ172 & YZ172 & $2021 / 8 / 3$ & $2021 / 8 / 14$ & II & Moderate & OPS & YZ & multiplex-pcr & Illumina & 36 & 37 & $2,395,738$ & $98.56 \%$ & 8997.583 \\
\hline YZ175 & YZ175 & $2021 / 8 / 4$ & $2021 / 8 / 5$ & II & Moderate & OPS & YZ & multiplex-pcr & Illumina & 27 & 29 & $1,858,767$ & $99.88 \%$ & 7435.49 \\
\hline YZ178 & YZ178 & $2021 / 8 / 3$ & $2021 / 8 / 4$ & II & Moderate & NPS & YZ & multiplex-pcr & $\mathrm{TF}$ & 25.5 & 24 & $2,195,424$ & $99.85 \%$ & 9906.395 \\
\hline YZ181 & YZ181 & 2021/8/1 & $2021 / 8 / 11$ & II & Mild & OPS & YZ & multiplex-pcr & BGI & 33 & 33 & 175,226 & $99.89 \%$ & 570.4045 \\
\hline
\end{tabular}




\begin{tabular}{|c|c|c|c|c|c|c|c|c|c|c|c|c|c|c|}
\hline YZ182 & YZ182 & $2021 / 7 / 29$ & $2021 / 8 / 11$ & $\mathrm{NE}$ & Moderate & NPS & YZ & multiplex-pcr & Illumina & 26 & 28 & $21,147,871$ & $99.90 \%$ & 83632.72 \\
\hline YZ187 & YZ187 & $2021 / 8 / 3$ & $2021 / 8 / 11$ & II & Moderate & NPS & YZ & multiplex-pcr & Illumina & 34 & 34 & $11,843,814$ & $99.82 \%$ & 41502.75 \\
\hline YZ188 & YZ188 & $2021 / 7 / 31$ & 2021/8/11 & II & Severe & NPS & YZ & multiplex-pcr & Illumina & 32 & 32 & $6,387,839$ & $99.82 \%$ & 23724.33 \\
\hline YZ192 & YZ192 & $2021 / 7 / 31$ & $2021 / 8 / 11$ & II & Moderate & NPS & YZ & multiplex-pcr & Illumina & 28 & 28 & $40,771,872$ & $99.87 \%$ & 156992.8 \\
\hline YZ196 & YZ196 & $2021 / 8 / 2$ & 2021/8/6 & II & Moderate & NPS & YZ & multiplex-pcr & Illumina & 37 & 39 & 520,905 & $98.99 \%$ & 1957.041 \\
\hline YZ198 & YZ198 & $2021 / 7 / 26$ & $2021 / 8 / 2$ & $\mathrm{NE}$ & Critical & NPS & YZ & multiplex-pcr & BGI & 20 & 21 & $13,806,372$ & $99.85 \%$ & 33115.98 \\
\hline YZ200 & YZ200 & $2021 / 7 / 31$ & $2021 / 8 / 2$ & II & Severe & NPS & YZ & multiplex-pcr & BGI & 34 & 32 & 128,528 & $99.74 \%$ & 310.6324 \\
\hline YZ202 & YZ202 & $2021 / 8 / 4$ & $2021 / 8 / 6$ & II & Moderate & NPS & YZ & multiplex-pcr & Illumina & 34 & 35 & $1,748,828$ & $99.79 \%$ & 6504.53 \\
\hline YZ204 & YZ204 & $2021 / 7 / 31$ & 2021/8/6 & II & Severe & NPS & YZ & multiplex-pcr & Illumina & 38 & 37 & $28,064,559$ & $99.94 \%$ & 107814.6 \\
\hline YZ210 & YZ210 & $2021 / 8 / 4$ & $2021 / 8 / 6$ & II & Moderate & OPS & YZ & multiplex-pcr & Illumina & 26 & 26 & $1,057,775$ & $99.56 \%$ & 3941.723 \\
\hline YZ211 & YZ211 & 2021/8/1 & 2021/8/6 & II & Moderate & OPS & YZ & multiplex-pcr & Illumina & 33 & 34 & $1,008,907$ & $99.36 \%$ & 3957.272 \\
\hline YZ212 & YZ212 & $2021 / 8 / 4$ & 2021/8/6 & II & Moderate & OPS & YZ & multiplex-pcr & Illumina & 28 & 27 & $1,902,567$ & $99.71 \%$ & 7617.377 \\
\hline YZ214 & YZ214 & $2021 / 8 / 5$ & 2021/8/6 & II & Mild & OPS & YZ & multiplex-pcr & Illumina & 32 & 33 & $1,111,334$ & $99.44 \%$ & 4294.378 \\
\hline YZ215 & YZ215 & $2021 / 8 / 3$ & 2021/8/6 & II & Moderate & OPS & YZ & multiplex-pcr & Illumina & 34 & 34 & $2,035,002$ & $99.50 \%$ & 7912.416 \\
\hline YZ224 & YZ224 & $2021 / 8 / 5$ & 2021/8/11 & II & Moderate & OPS & YZ & multiplex-pcr & Illumina & 33 & 33 & $2,073,165$ & $99.52 \%$ & 8037.804 \\
\hline YZ227 & YZ227 & $2021 / 8 / 4$ & $2021 / 9 / 23$ & $\mathrm{NE}$ & Moderate & NPS & YZ & multiplex-pcr & Illumina & 32 & 33 & $2,045,507$ & $99.74 \%$ & 6873.464 \\
\hline YZ229 & YZ229 & $2021 / 7 / 30$ & $2021 / 8 / 2$ & $\mathrm{NE}$ & Critical & NPS & YZ & multiplex-pcr & BGI & 27 & 26 & $12,727,899$ & $99.87 \%$ & 31022.64 \\
\hline YZ230 & YZ230 & $2021 / 8 / 3$ & $2021 / 8 / 11$ & II & Severe & NPS & YZ & multiplex-pcr & Illumina & 30 & 30 & $8,289,250$ & $99.88 \%$ & 32209.97 \\
\hline YZ232 & YZ232 & $2021 / 8 / 5$ & $2021 / 8 / 6$ & II & Moderate & NPS & YZ & multiplex-pcr & Illumina & 34 & 34 & $1,143,673$ & $99.83 \%$ & 4428.969 \\
\hline YZ234 & YZ234 & $2021 / 8 / 4$ & 2021/8/6 & II & Moderate & NPS & YZ & multiplex-pcr & Illumina & 27 & 24 & 981,941 & $99.76 \%$ & 3879.749 \\
\hline YZ235 & YZ235 & $2021 / 8 / 5$ & 2021/8/6 & II & Critical & NPS & YZ & multiplex-pcr & Illumina & 25 & 23 & 482,974 & $99.57 \%$ & 1908.759 \\
\hline YZ236 & YZ236 & $2021 / 8 / 4$ & $2021 / 8 / 19$ & II & Moderate & NPS & YZ & multiplex-pcr & Illumina & 33 & 32 & $1,218,309$ & $99.85 \%$ & 4509.005 \\
\hline YZ240 & YZ240 & $2021 / 8 / 5$ & 2021/8/19 & II & Moderate & NPS & YZ & multiplex-pcr & Illumina & 33 & 33 & $6,920,826$ & $99.84 \%$ & 22568.44 \\
\hline YZ242 & YZ242 & $2021 / 8 / 5$ & $2021 / 8 / 11$ & II & Mild & NPS & YZ & multiplex-pcr & Illumina & 24 & 24 & $23,152,212$ & $99.94 \%$ & 92512.51 \\
\hline YZ249 & YZ249 & $2021 / 8 / 4$ & 2021/8/6 & II & Moderate & OPS & YZ & multiplex-pcr & Illumina & 22 & 21 & $1,351,732$ & $99.56 \%$ & 5197.615 \\
\hline YZ251 & YZ251 & $2021 / 8 / 5$ & $2021 / 8 / 18$ & II & Mild & OPS & $\mathrm{YZ}$ & multiplex-pcr & Illumina & 30 & 32 & $1,862,987$ & $99.90 \%$ & 6984.405 \\
\hline YZ254 & YZ254 & $2021 / 8 / 5$ & 2021/8/11 & II & Severe & NPS & YZ & multiplex-pcr & Illumina & 31 & 31 & $10,806,436$ & $99.93 \%$ & 40182.28 \\
\hline YZ256 & YZ256 & $2021 / 8 / 4$ & $2021 / 8 / 18$ & II & Moderate & OPS & YZ & multiplex-pcr & Illumina & 33 & 34 & 295,292 & $99.24 \%$ & 1116.229 \\
\hline YZ265 & YZ265 & $2021 / 8 / 5$ & $2021 / 8 / 11$ & II & Moderate & NPS & YZ & multiplex-pcr & BGI & 30 & 30 & 373,658 & $99.89 \%$ & 1246.48 \\
\hline
\end{tabular}




\begin{tabular}{|c|c|c|c|c|c|c|c|c|c|c|c|c|c|c|}
\hline YZ266 & YZ266 & $2021 / 8 / 5$ & $2021 / 8 / 11$ & II & Moderate & NPS & YZ & multiplex-pcr & BGI & 18 & 18 & 352,353 & $99.94 \%$ & 1147.764 \\
\hline YZ268 & YZ268 & $2021 / 8 / 5$ & $2021 / 8 / 11$ & II & Moderate & NPS & YZ & multiplex-pcr & BGI & 35 & 35 & 62,419 & $99.47 \%$ & 204.7944 \\
\hline YZ271 & YZ271 & $2021 / 8 / 5$ & $2021 / 8 / 19$ & II & Mild & OPS & YZ & multiplex-pcr & Illumina & 31 & 31 & $3,604,361$ & $99.87 \%$ & 13824.5 \\
\hline YZ272 & YZ272 & $2021 / 8 / 5$ & $8 / 18 / 2021$ & II & Mild & OPS & YZ & multiplex-pcr & Illumina & 32 & 34 & $18,416,391$ & $99.93 \%$ & 68807.29 \\
\hline YZ275 & YZ275 & $2021 / 8 / 6$ & 2021/8/7 & II & Moderate & NPS & YZ & multiplex-pcr & Illumina & 19 & 19 & 643,515 & $99.93 \%$ & 2149.275 \\
\hline YZ278 & YZ278 & $2021 / 8 / 1$ & 2021/8/19 & II & Moderate & NPS & YZ & multiplex-pcr & Illumina & 26 & 27 & $10,281,151$ & $99.93 \%$ & 39609.08 \\
\hline YZ279 & YZ279 & $2021 / 8 / 5$ & $2021 / 8 / 11$ & II & Moderate & NPS & YZ & multiplex-pcr & Illumina & 33 & 33 & 273,118 & $99.56 \%$ & 1024.564 \\
\hline YZ280 & YZ280 & $2021 / 8 / 5$ & $2021 / 8 / 19$ & II & Moderate & NPS & YZ & multiplex-pcr & Illumina & 23 & 25 & $17,762,709$ & $99.94 \%$ & 66302.55 \\
\hline YZ281 & YZ281 & $2021 / 8 / 5$ & $2021 / 8 / 18$ & II & Moderate & NPS & YZ & multiplex-pcr & Illumina & 29 & 29 & $11,088,918$ & $99.94 \%$ & 41678.39 \\
\hline YZ282 & YZ282 & $2021 / 8 / 6$ & 2021/8/19 & II & Moderate & NPS & YZ & multiplex-pcr & Illumina & 14 & 14 & $16,938,798$ & $99.94 \%$ & 64715.86 \\
\hline YZ283 & YZ283 & $2021 / 8 / 5$ & $2021 / 8 / 19$ & II & Moderate & NPS & YZ & multiplex-pcr & Illumina & 17 & 17 & $14,419,736$ & $99.94 \%$ & 56103.2 \\
\hline YZ284 & YZ284 & $2021 / 8 / 5$ & $2021 / 8 / 18$ & II & Moderate & NPS & YZ & multiplex-pcr & Illumina & 16 & 17 & $21,164,008$ & $99.94 \%$ & 73816.79 \\
\hline YZ285 & YZ285 & $2021 / 8 / 6$ & 2021/8/11 & II & Moderate & NPS & YZ & multiplex-pcr & Illumina & 33 & 33 & $1,530,147$ & $99.85 \%$ & 5789.582 \\
\hline YZ286 & YZ286 & 2021/8/7 & $2021 / 8 / 19$ & II & Moderate & NPS & YZ & multiplex-pcr & Illumina & 19 & 19 & $14,431,679$ & $99.93 \%$ & 55292.96 \\
\hline YZ288 & YZ288 & $2021 / 8 / 5$ & 2021/8/11 & II & Severe & NPS & YZ & multiplex-pcr & Illumina & 32 & 32 & $2,394,309$ & $99.83 \%$ & 9104.587 \\
\hline YZ289 & YZ289 & $2021 / 8 / 5$ & 2021/8/7 & II & Moderate & NPS & YZ & multiplex-pcr & Illumina & 31 & 28 & 684,518 & $99.68 \%$ & 2284.642 \\
\hline YZ290 & YZ290 & $2021 / 8 / 6$ & $2021 / 8 / 11$ & II & Moderate & NPS & YZ & multiplex-pcr & Illumina & 27 & 27 & $3,446,589$ & $99.68 \%$ & 12361.52 \\
\hline YZ291 & YZ291 & $2021 / 8 / 6$ & $2021 / 8 / 18$ & II & Moderate & NPS & YZ & multiplex-pcr & Illumina & 29 & 29 & $2,274,406$ & $99.59 \%$ & 8934.633 \\
\hline YZ298 & YZ298 & $2021 / 8 / 6$ & $2021 / 8 / 11$ & II & Severe & NPS & YZ & multiplex-pcr & Illumina & 31 & 31 & $19,327,073$ & $99.94 \%$ & 73727.15 \\
\hline YZ303 & YZ303 & $2021 / 8 / 5$ & $2021 / 8 / 11$ & II & Moderate & NPS & YZ & multiplex-pcr & Illumina & 33 & 33 & $33,984,348$ & $99.88 \%$ & 121622.1 \\
\hline YZ304 & YZ304 & $2021 / 8 / 6$ & $2021 / 8 / 11$ & II & Moderate & NPS & YZ & multiplex-pcr & Illumina & 26 & 28 & $24,080,266$ & $99.87 \%$ & 95047.11 \\
\hline YZ307 & YZ307 & $2021 / 8 / 2$ & $2021 / 8 / 19$ & II & Moderate & NPS & YZ & multiplex-pcr & Illumina & 24 & 25 & $13,970,217$ & $99.93 \%$ & 52657.07 \\
\hline YZ308 & YZ308 & $2021 / 8 / 4$ & 2021/8/11 & II & Moderate & NPS & YZ & multiplex-pcr & Illumina & 23 & 24 & $2,005,515$ & $99.86 \%$ & 7927.65 \\
\hline YZ309 & YZ309 & $2021 / 8 / 6$ & $2021 / 8 / 11$ & II & Moderate & NPS & YZ & multiplex-pcr & Illumina & 27 & 28 & $1,890,571$ & $99.84 \%$ & 7555.75 \\
\hline YZ310 & YZ310 & $2021 / 8 / 6$ & $2021 / 8 / 11$ & II & Moderate & NPS & YZ & multiplex-pcr & Illumina & 22 & 23 & $1,891,507$ & $99.93 \%$ & 6243.864 \\
\hline YZ311 & YZ311 & $2021 / 8 / 7$ & $2021 / 8 / 18$ & II & Moderate & NPS & $\mathrm{YZ}$ & multiplex-pcr & Illumina & 27 & 28 & $6,626,249$ & $99.88 \%$ & 25058.76 \\
\hline YZ315 & YZ315 & $2021 / 8 / 5$ & 2021/8/11 & II & Moderate & OPS & YZ & multiplex-pcr & Illumina & 28 & 28.2 & $5,013,865$ & $99.90 \%$ & 18425.42 \\
\hline YZ319 & YZ319 & $2021 / 8 / 7$ & $2021 / 8 / 8$ & II & Mild & NPS & YZ & multiplex-pcr & Illumina & 27 & 26 & 193,330 & $99.74 \%$ & 784.58 \\
\hline YZ323 & YZ323 & 2021/8/7 & $2021 / 8 / 8$ & II & Moderate & NPS & YZ & multiplex-pcr & Illumina & 26 & 26 & 369,023 & $99.74 \%$ & 1455.258 \\
\hline
\end{tabular}




\begin{tabular}{|c|c|c|c|c|c|c|c|c|c|c|c|c|c|c|}
\hline YZ326 & YZ326 & $2021 / 8 / 7$ & $2021 / 8 / 11$ & II & Moderate & NPS & YZ & multiplex-pcr & Illumina & 29 & 30 & $3,056,235$ & $99.93 \%$ & 11225.91 \\
\hline YZ328 & YZ328 & 2021/8/7 & $2021 / 8 / 11$ & II & Moderate & NPS & YZ & multiplex-pcr & Illumina & 21 & 22 & $1,731,558$ & $99.94 \%$ & 6924.178 \\
\hline YZ329 & YZ329 & 2021/8/7 & 2021/8/11 & II & Moderate & NPS & YZ & multiplex-pcr & Illumina & 32 & 35 & 350,607 & $99.75 \%$ & 1320.504 \\
\hline YZ330 & YZ330 & $2021 / 8 / 4$ & $2021 / 8 / 19$ & II & Moderate & NPS & YZ & multiplex-pcr & Illumina & 29 & 29 & $16,093,247$ & $99.86 \%$ & 60984.61 \\
\hline YZ331 & YZ331 & $2021 / 8 / 2$ & 2021/8/11 & II & Moderate & NPS & YZ & multiplex-pcr & Illumina & 30 & 30 & $1,533,060$ & $99.86 \%$ & 5568.201 \\
\hline YZ334 & YZ334 & $2021 / 8 / 7$ & $2021 / 8 / 8$ & II & Moderate & NPS & YZ & multiplex-pcr & Illumina & 25 & 25 & 189,594 & $99.56 \%$ & 759.6693 \\
\hline YZ338 & YZ338 & $2021 / 8 / 4$ & $2021 / 8 / 11$ & II & Moderate & NPS & YZ & multiplex-pcr & Illumina & 20 & 22 & $31,980,006$ & $99.94 \%$ & 124808.9 \\
\hline YZ339 & YZ339 & $2021 / 8 / 1$ & $2021 / 8 / 11$ & II & Mild & NPS & YZ & multiplex-pcr & Illumina & 32 & 32 & $7,882,454$ & $99.82 \%$ & 29740.9 \\
\hline YZ340 & YZ340 & 2021/8/7 & $2021 / 8 / 8$ & II & Mild & NPS & YZ & multiplex-pcr & Illumina & 22 & 20 & 576,961 & $99.77 \%$ & 2322.174 \\
\hline YZ341 & YZ341 & $2021 / 8 / 8$ & $2021 / 8 / 8$ & $\mathrm{CI}$ & Moderate & NPS & YZ & multiplex-pcr & Illumina & 22 & 21 & 607,944 & $99.58 \%$ & 2444.163 \\
\hline YZ342 & YZ342 & $2021 / 8 / 5$ & $2021 / 8 / 18$ & II & Moderate & NPS & YZ & multiplex-pcr & Illumina & 30 & 30 & $7,351,180$ & $99.88 \%$ & 27594.85 \\
\hline YZ343 & YZ343 & $2021 / 7 / 25$ & $2021 / 8 / 2$ & $\mathrm{NE}$ & Moderate & OPS & YZ & multiplex-pcr & BGI & 32 & 30 & 395,850 & $99.83 \%$ & 954.9447 \\
\hline YZ345 & YZ345 & 2021/8/7 & 2021/8/9 & II & Moderate & NPS & YZ & multiplex-pcr & Illumina & 34 & 38 & $1,336,398$ & $99.94 \%$ & 5253.582 \\
\hline YZ348 & YZ348 & 2021/8/7 & $2021 / 8 / 11$ & II & Moderate & NPS & YZ & multiplex-pcr & Illumina & 20 & 20 & $2,809,689$ & $99.94 \%$ & 11277.17 \\
\hline YZ350 & YZ350 & 2021/8/7 & 2021/8/11 & II & Moderate & NPS & YZ & multiplex-pcr & Illumina & 17 & 19 & $16,273,985$ & $99.94 \%$ & 64186.88 \\
\hline YZ351 & YZ351 & $2021 / 8 / 8$ & $2021 / 8 / 18$ & $\mathrm{CI}$ & Moderate & NPS & YZ & multiplex-pcr & Illumina & 33 & 34 & $2,479,611$ & $99.07 \%$ & 9487.248 \\
\hline YZ352 & YZ352 & $2021 / 8 / 8$ & $2021 / 8 / 11$ & $\mathrm{CI}$ & Mild & NPS & YZ & multiplex-pcr & Illumina & 30 & 30 & $30,167,034$ & $99.85 \%$ & 115703.4 \\
\hline YZ353 & YZ353 & $2021 / 8 / 6$ & 2021/8/9 & II & Moderate & NPS & YZ & multiplex-pcr & Illumina & 28 & 28 & $2,774,725$ & $99.94 \%$ & 11161.65 \\
\hline YZ354 & YZ354 & $2021 / 8 / 7$ & 2021/8/9 & II & Moderate & NPS & YZ & multiplex-pcr & Illumina & 34 & 34 & $2,138,256$ & $98.99 \%$ & 7975.334 \\
\hline YZ355 & YZ355 & 2021/8/6 & $2021 / 8 / 11$ & II & Moderate & NPS & YZ & multiplex-pcr & Illumina & 17 & 20 & $23,935,317$ & $99.94 \%$ & 91840.22 \\
\hline YZ356 & YZ356 & $2021 / 8 / 7$ & 2021/8/9 & II & Mild & NPS & YZ & multiplex-pcr & Illumina & 32 & 32 & $5,717,220$ & $99.90 \%$ & 19674.22 \\
\hline YZ357 & YZ357 & $2021 / 8 / 4$ & $2021 / 8 / 11$ & II & Mild & OPS & YZ & multiplex-pcr & Illumina & 32 & 33 & $1,362,037$ & $99.81 \%$ & 5324.623 \\
\hline YZ358 & YZ358 & $2021 / 8 / 6$ & 2021/8/9 & II & Moderate & OPS & YZ & multiplex-pcr & Illumina & 27 & 27 & $3,313,288$ & $99.92 \%$ & 13348.19 \\
\hline YZ360 & YZ360 & $2021 / 8 / 7$ & 2021/8/9 & II & Moderate & OPS & YZ & multiplex-pcr & Illumina & 30 & 32 & $1,670,497$ & $99.82 \%$ & 5663.923 \\
\hline YZ362 & YZ362 & $2021 / 8 / 5$ & $2021 / 8 / 11$ & II & Moderate & NPS & YZ & multiplex-pcr & Illumina & 24 & 24 & $30,911,678$ & $99.99 \%$ & 123339.4 \\
\hline YZ363 & YZ363 & $2021 / 8 / 4$ & 2021/8/11 & II & Critical & NPS & $\mathrm{YZ}$ & multiplex-pcr & Illumina & 20 & 20 & $21,990,916$ & $99.94 \%$ & 84499.94 \\
\hline YZ364 & YZ364 & 2021/8/7 & 2021/8/11 & II & Moderate & NPS & YZ & multiplex-pcr & Illumina & 30 & 31 & $2,473,670$ & $99.93 \%$ & 9687.334 \\
\hline YZ365 & YZ365 & 2021/8/7 & $2021 / 8 / 11$ & II & Moderate & NPS & YZ & multiplex-pcr & Illumina & 21 & 23 & $1,407,252$ & $99.90 \%$ & 5587.949 \\
\hline YZ366 & YZ366 & 2021/8/7 & 2021/8/9 & II & Moderate & NPS & YZ & multiplex-pcr & Illumina & 30 & 30 & $2,858,406$ & $99.74 \%$ & 11499.21 \\
\hline
\end{tabular}




\begin{tabular}{|c|c|c|c|c|c|c|c|c|c|c|c|c|c|c|}
\hline YZ367 & YZ367 & $2021 / 8 / 7$ & $2021 / 8 / 9$ & II & Mild & OPS & $\mathrm{YZ}$ & multiplex-pcr & Illumina & 30 & 30 & $3,016,439$ & $99.93 \%$ & 12120.94 \\
\hline YZ368 & YZ368 & $2021 / 8 / 8$ & 2021/8/9 & CI & Moderate & NPS & YZ & multiplex-pcr & Illumina & 33 & 33 & $3,379,807$ & $99.83 \%$ & 13612.13 \\
\hline YZ374 & YZ374 & $2021 / 8 / 7$ & 2021/8/11 & II & Mild & OPS & YZ & multiplex-pcr & Illumina & 32 & 33 & $3,008,852$ & $99.86 \%$ & 11601.56 \\
\hline YZ375 & YZ375 & $2021 / 8 / 6$ & $2021 / 8 / 11$ & II & Moderate & NPS & YZ & multiplex-pcr & Illumina & 24 & 24 & $37,152,195$ & $99.94 \%$ & 140957.5 \\
\hline YZ376 & YZ376 & $2021 / 8 / 7$ & $2021 / 8 / 12$ & II & Mild & OPS & YZ & multiplex-pcr & Illumina & 26 & 26 & $1,456,716$ & $99.29 \%$ & 5837.734 \\
\hline YZ378 & YZ378 & $2021 / 8 / 8$ & 2021/8/11 & CI & Moderate & OPS & YZ & multiplex-pcr & Illumina & 36 & 35 & $1,734,739$ & $99.72 \%$ & 6971.058 \\
\hline YZ380 & YZ380 & $2021 / 8 / 4$ & 2021/8/11 & II & Moderate & NPS & YZ & multiplex-pcr & Illumina & 20 & 20 & $19,612,173$ & $99.94 \%$ & 75911.87 \\
\hline YZ381 & YZ381 & $2021 / 8 / 8$ & $2021 / 8 / 11$ & $\mathrm{CI}$ & Mild & OPS & YZ & multiplex-pcr & Illumina & 27 & 28 & $1,963,325$ & $99.90 \%$ & 7867.678 \\
\hline YZ383 & YZ383 & $2021 / 8 / 4$ & $2021 / 8 / 11$ & II & Moderate & NPS & YZ & multiplex-pcr & Illumina & 24 & 24 & $23,052,751$ & $99.94 \%$ & 91010.4 \\
\hline YZ385 & YZ385 & $2021 / 8 / 8$ & $2021 / 8 / 11$ & CI & Moderate & NPS & YZ & multiplex-pcr & Illumina & 19 & 20 & $14,918,001$ & $99.94 \%$ & 58514.6 \\
\hline YZ386 & YZ386 & $2021 / 8 / 8$ & 2021/8/11 & $\mathrm{CI}$ & Severe & NPS & YZ & multiplex-pcr & Illumina & 26 & 28 & $24,948,522$ & $99.94 \%$ & 97426.32 \\
\hline YZ387 & YZ387 & $2021 / 8 / 7$ & $2021 / 8 / 12$ & II & Moderate & OPS & YZ & multiplex-pcr & Illumina & 29 & 28 & $1,225,594$ & $99.13 \%$ & 4899.398 \\
\hline YZ388 & YZ388 & $2021 / 8 / 8$ & $2021 / 8 / 10$ & CI & Mild & OPS & YZ & multiplex-pcr & $\mathrm{TF}$ & 29 & 28 & 763,636 & $99.77 \%$ & 4627.807 \\
\hline YZ391 & YZ391 & $2021 / 8 / 8$ & $2021 / 8 / 11$ & $\mathrm{CI}$ & Moderate & NPS & YZ & multiplex-pcr & Illumina & 16 & 18 & $17,871,746$ & $99.94 \%$ & 70134.84 \\
\hline YZ392 & YZ392 & $2021 / 8 / 5$ & $2021 / 8 / 11$ & II & Moderate & NPS & YZ & multiplex-pcr & Illumina & 26 & 27 & $20,039,407$ & $99.94 \%$ & 79100.37 \\
\hline YZ393 & YZ393 & $2021 / 8 / 8$ & $2021 / 8 / 11$ & CI & Moderate & NPS & YZ & multiplex-pcr & Illumina & 24 & 26 & $26,345,467$ & $99.84 \%$ & 103677.7 \\
\hline YZ394 & YZ394 & $2021 / 8 / 2$ & $2021 / 8 / 11$ & II & Moderate & NPS & YZ & multiplex-pcr & Illumina & 32 & 33 & $38,343,842$ & $99.88 \%$ & 134896.5 \\
\hline YZ395 & YZ395 & 2021/8/9 & $2021 / 8 / 11$ & $\mathrm{CI}$ & Mild & NPS & YZ & multiplex-pcr & Illumina & 22 & 25 & $22,988,304$ & $99.93 \%$ & 88592.28 \\
\hline YZ396 & YZ396 & $2021 / 8 / 8$ & $2021 / 8 / 11$ & $\mathrm{CI}$ & Severe & NPS & YZ & multiplex-pcr & Illumina & 24 & 25 & $23,320,978$ & $99.86 \%$ & 87800.49 \\
\hline YZ397 & YZ397 & $2021 / 8 / 8$ & $2021 / 8 / 10$ & CI & Moderate & OPS & YZ & multiplex-pcr & $\mathrm{TF}$ & 20 & 20 & $2,652,447$ & $99.91 \%$ & 17511.01 \\
\hline YZ398 & YZ398 & $2021 / 8 / 8$ & $2021 / 8 / 11$ & CI & Moderate & OPS & YZ & multiplex-pcr & Illumina & 30 & 31 & $1,515,258$ & $99.93 \%$ & 5865.973 \\
\hline YZ399 & YZ399 & $2021 / 8 / 7$ & $2021 / 8 / 10$ & II & Moderate & OPS & YZ & multiplex-pcr & $\mathrm{TF}$ & 21 & 20 & $2,529,031$ & $99.89 \%$ & 16709.9 \\
\hline YZ400 & YZ400 & $2021 / 8 / 8$ & $2021 / 8 / 10$ & $\mathrm{CI}$ & Moderate & OPS & YZ & multiplex-pcr & $\mathrm{TF}$ & 20 & 20 & $2,231,102$ & $99.87 \%$ & 14778.87 \\
\hline YZ401 & YZ401 & $2021 / 8 / 2$ & $2021 / 8 / 10$ & II & Moderate & OPS & YZ & multiplex-pcr & $\mathrm{TF}$ & 30 & 30 & 357,172 & $99.77 \%$ & 2278.9 \\
\hline YZ402 & YZ402 & $2021 / 8 / 9$ & $2021 / 8 / 18$ & CI & Moderate & OPS & YZ & multiplex-pcr & Illumina & 27 & 29 & $4,306,711$ & $99.93 \%$ & 15569.48 \\
\hline YZ403 & YZ403 & $2021 / 8 / 6$ & $2021 / 8 / 10$ & II & Moderate & OPS & YZ & multiplex-pcr & $\mathrm{TF}$ & 25 & 25 & $1,156,317$ & $99.77 \%$ & 7172.973 \\
\hline YZ404 & YZ404 & $2021 / 8 / 10$ & $2021 / 8 / 11$ & $\mathrm{CI}$ & Moderate & OPS & YZ & multiplex-pcr & BGI & 25 & 25 & $1,224,800$ & $99.90 \%$ & 4085.871 \\
\hline YZ405 & YZ405 & $2021 / 8 / 9$ & 2021/8/11 & $\mathrm{CI}$ & Mild & OPS & YZ & multiplex-pcr & BGI & 26 & 26 & $1,453,957$ & $99.89 \%$ & 4852.018 \\
\hline YZ406 & YZ406 & 2021/8/7 & $2021 / 8 / 11$ & II & Moderate & OPS & YZ & multiplex-pcr & BGI & 27 & 27 & $1,214,804$ & $99.89 \%$ & 4052.508 \\
\hline
\end{tabular}




\begin{tabular}{|c|c|c|c|c|c|c|c|c|c|c|c|c|c|c|}
\hline $\begin{array}{l}\mathrm{YZ} 407 \\
\end{array}$ & YZ407 & $2021 / 8 / 4$ & $2021 / 8 / 10$ & II & Mild & NPS & YZ & multiplex-pcr & TF & 32 & 30 & 412,336 & $99.77 \%$ & 2489.916 \\
\hline YZ408 & YZ408 & $2021 / 8 / 10$ & $2021 / 8 / 11$ & $\mathrm{CI}$ & Severe & NPS & YZ & multiplex-pcr & Illumina & 16 & 18 & $28,494,535$ & $99.94 \%$ & 112487.6 \\
\hline YZ409 & YZ409 & $2021 / 8 / 8$ & $2021 / 8 / 10$ & $\mathrm{CI}$ & Moderate & OPS & YZ & multiplex-pcr & TF & 27 & 25 & $2,508,870$ & $99.81 \%$ & 15970.54 \\
\hline YZ410 & YZ410 & $2021 / 8 / 7$ & $2021 / 8 / 10$ & II & Moderate & OPS & $\mathrm{YZ}$ & multiplex-pcr & TF & 25 & 24 & $1,824,349$ & $99.85 \%$ & 12249.53 \\
\hline YZ411 & YZ411 & $2021 / 8 / 5$ & $2021 / 8 / 11$ & II & Moderate & NPS & YZ & multiplex-pcr & BGI & 31 & 31 & 617,533 & $99.89 \%$ & 2060.022 \\
\hline YZ412 & YZ412 & $2021 / 8 / 8$ & $2021 / 8 / 10$ & $\mathrm{CI}$ & Moderate & NPS & YZ & multiplex-pcr & TF & 24 & 24 & $1,734,326$ & $99.84 \%$ & 11104.85 \\
\hline YZ413 & YZ413 & $2021 / 8 / 6$ & $2021 / 8 / 10$ & II & Moderate & NPS & YZ & multiplex-pcr & TF & 32 & 30 & 214,632 & $99.77 \%$ & 1359.505 \\
\hline YZ414 & YZ414 & 2021/8/9 & $2021 / 8 / 10$ & $\mathrm{CI}$ & Mild & NPS & YZ & multiplex-pcr & $\mathrm{TF}$ & 27 & 27 & $1,667,594$ & $99.78 \%$ & 10742.46 \\
\hline YZ415 & YZ415 & 2021/8/9 & $2021 / 8 / 10$ & $\mathrm{CI}$ & Mild & NPS & YZ & multiplex-pcr & TF & 28 & 28 & $1,153,930$ & $99.82 \%$ & 7174.557 \\
\hline YZ416 & YZ416 & 2021/8/9 & $2021 / 8 / 10$ & $\mathrm{CI}$ & Moderate & NPS & YZ & multiplex-pcr & TF & 20 & 22 & $2,983,963$ & $99.82 \%$ & 20091.1 \\
\hline YZ417 & YZ417 & $2021 / 8 / 8$ & $2021 / 8 / 10$ & $\mathrm{CI}$ & Mild & NPS & YZ & multiplex-pcr & TF & 18 & 20 & $1,775,859$ & $99.83 \%$ & 11657.68 \\
\hline YZ418 & YZ418 & $2021 / 8 / 8$ & $2021 / 8 / 11$ & $\mathrm{CI}$ & Mild & NPS & YZ & multiplex-pcr & Illumina & 18 & 19 & $1,899,110$ & $99.94 \%$ & 7672.136 \\
\hline YZ419 & YZ419 & 2021/8/9 & 2021/8/11 & $\mathrm{CI}$ & Mild & NPS & YZ & multiplex-pcr & Illumina & 33 & 34 & 507,801 & $98.89 \%$ & 2010.902 \\
\hline YZ420 & YZ420 & 2021/8/7 & $2021 / 8 / 11$ & II & Moderate & NPS & YZ & multiplex-pcr & Illumina & 23 & 25 & $1,942,947$ & $99.84 \%$ & 7820.37 \\
\hline YZ422 & YZ422 & $2021 / 8 / 4$ & $2021 / 8 / 8$ & II & Moderate & NPS & YZ & multiplex-pcr & Illumina & 30 & 29 & 102,863 & $99.47 \%$ & 417.3331 \\
\hline YZ424 & YZ424 & 2021/8/9 & 2021/8/11 & $\mathrm{CI}$ & Moderate & NPS & YZ & multiplex-pcr & Illumina & 20 & 21 & $2,281,297$ & $99.93 \%$ & 8413.484 \\
\hline YZ425 & YZ425 & 2021/8/9 & $2021 / 8 / 10$ & $\mathrm{CI}$ & Moderate & NPS & YZ & multiplex-pcr & TF & 31 & 32 & $2,101,102$ & $99.78 \%$ & 14234.28 \\
\hline YZ426 & YZ426 & $2021 / 8 / 8$ & $2021 / 8 / 10$ & $\mathrm{CI}$ & Moderate & OPS & YZ & multiplex-pcr & TF & 30 & 29 & $2,229,780$ & $99.81 \%$ & 14772.75 \\
\hline YZ427 & YZ427 & 2021/8/9 & $2021 / 8 / 10$ & $\mathrm{CI}$ & Moderate & NPS & YZ & multiplex-pcr & Illumina & 35 & 30 & $3,730,192$ & $99.88 \%$ & 13639.17 \\
\hline YZ428 & YZ428 & $2021 / 8 / 10$ & $2021 / 8 / 10$ & $\mathrm{CI}$ & Mild & NPS & YZ & multiplex-pcr & Illumina & 24 & 24 & $1,656,503$ & $99.81 \%$ & 6658.233 \\
\hline YZ429 & YZ429 & 2021/8/9 & $2021 / 8 / 10$ & $\mathrm{CI}$ & Moderate & NPS & YZ & multiplex-pcr & Illumina & 35 & 34 & $3,354,830$ & $99.94 \%$ & 12838.89 \\
\hline YZ430 & YZ430 & 2021/8/9 & $2021 / 8 / 10$ & $\mathrm{CI}$ & Mild & NPS & YZ & multiplex-pcr & TF & 26 & 26 & $1,974,665$ & $99.85 \%$ & 13287.63 \\
\hline YZ431 & YZ431 & 2021/8/9 & $2021 / 8 / 10$ & $\mathrm{CI}$ & Moderate & NPS & YZ & multiplex-pcr & $\mathrm{TF}$ & 31 & 30 & $2,288,925$ & $99.79 \%$ & 15475.22 \\
\hline YZ433 & YZ433 & 2021/8/7 & $2021 / 8 / 10$ & II & Moderate & NPS & YZ & multiplex-pcr & TF & 22 & 24 & $2,458,768$ & $99.82 \%$ & 15928.28 \\
\hline YZ434 & YZ434 & 2021/8/9 & $2021 / 8 / 10$ & $\mathrm{CI}$ & Moderate & NPS & YZ & multiplex-pcr & TF & 25 & 25 & $2,354,189$ & $99.75 \%$ & 15823.29 \\
\hline YZ435 & YZ435 & 2021/8/9 & $2021 / 8 / 10$ & $\mathrm{CI}$ & Severe & OPS & YZ & multiplex-pcr & $\mathrm{TF}$ & 25 & 26 & $2,017,316$ & $99.77 \%$ & 13390.97 \\
\hline YZ436 & YZ436 & 2021/8/7 & $2021 / 8 / 10$ & II & Mild & NPS & YZ & multiplex-pcr & Illumina & 30 & 30 & $2,467,858$ & $99.91 \%$ & 9915.299 \\
\hline YZ438 & YZ438 & $2021 / 8 / 4$ & $2021 / 8 / 18$ & II & Moderate & NPS & YZ & multiplex-pcr & Illumina & 24 & 25 & $1,854,648$ & $99.59 \%$ & 6975.67 \\
\hline YZ439 & YZ439 & 2021/8/9 & $2021 / 8 / 18$ & $\mathrm{CI}$ & Moderate & NPS & YZ & multiplex-pcr & Illumina & 30 & 30 & 697,645 & $98.89 \%$ & 2372.728 \\
\hline
\end{tabular}




\begin{tabular}{|c|c|c|c|c|c|c|c|c|c|c|c|c|c|c|}
\hline $\begin{array}{l}\mathrm{YZ} 440 \\
\end{array}$ & YZ440 & $2021 / 8 / 7$ & $2021 / 8 / 18$ & II & Severe & NPS & YZ & multiplex-pcr & Illumina & 23 & 24 & $9,925,468$ & $99.94 \%$ & 38033.45 \\
\hline YZ441 & YZ441 & $2021 / 8 / 5$ & $2021 / 8 / 10$ & II & Moderate & NPS & YZ & multiplex-pcr & TF & 30 & 30 & $2,011,151$ & $99.78 \%$ & 13634.75 \\
\hline YZ442 & YZ442 & 2021/8/7 & $2021 / 8 / 10$ & II & Moderate & NPS & YZ & multiplex-pcr & TF & 25 & 26 & $2,190,698$ & $99.78 \%$ & 14740.3 \\
\hline YZ443 & YZ443 & $2021 / 8 / 4$ & $2021 / 8 / 10$ & II & Moderate & NPS & YZ & multiplex-pcr & TF & 21 & 24 & $2,462,431$ & $99.79 \%$ & 16727.03 \\
\hline YZ444 & YZ444 & 2021/8/9 & $2021 / 8 / 12$ & CI & Moderate & NPS & YZ & multiplex-pcr & Illumina & 31 & 31 & $2,265,480$ & $99.58 \%$ & 9028.512 \\
\hline YZ446 & YZ446 & $2021 / 8 / 6$ & $2021 / 8 / 11$ & II & Moderate & OPS & YZ & multiplex-pcr & Illumina & 24 & 25 & $2,061,177$ & $99.86 \%$ & 8270.156 \\
\hline YZ448 & YZ448 & $2021 / 8 / 10$ & $2021 / 8 / 11$ & $\mathrm{CI}$ & Moderate & NPS & YZ & multiplex-pcr & Illumina & 25 & 26 & $25,731,971$ & $99.87 \%$ & 98132.75 \\
\hline YZ450 & YZ450 & 2021/8/9 & $2021 / 8 / 11$ & $\mathrm{CI}$ & Moderate & NPS & YZ & multiplex-pcr & Illumina & 18 & 20 & $17,833,578$ & $99.94 \%$ & 71029.37 \\
\hline YZ451 & YZ451 & 2021/8/9 & $2021 / 8 / 11$ & $\mathrm{CI}$ & Moderate & NPS & YZ & multiplex-pcr & Illumina & 18 & 20 & $21,514,559$ & $99.94 \%$ & 85342.05 \\
\hline YZ452 & YZ452 & $2021 / 8 / 8$ & $2021 / 8 / 10$ & $\mathrm{CI}$ & Mild & NPS & YZ & multiplex-pcr & TF & 27 & 27 & $1,573,036$ & $99.80 \%$ & 10191.64 \\
\hline YZ453 & YZ453 & $2021 / 8 / 5$ & $2021 / 8 / 11$ & II & Moderate & NPS & YZ & multiplex-pcr & Illumina & 24 & 26 & $19,218,774$ & $99.79 \%$ & 74832.61 \\
\hline YZ454 & YZ454 & $2021 / 8 / 10$ & $2021 / 8 / 11$ & $\mathrm{CI}$ & Moderate & NPS & YZ & multiplex-pcr & Illumina & 32 & 33 & $16,456,867$ & $99.83 \%$ & 60148.29 \\
\hline YZ455 & YZ455 & $2021 / 8 / 10$ & 2021/8/11 & $\mathrm{CI}$ & Mild & NPS & YZ & multiplex-pcr & Illumina & 20 & 20 & $20,465,691$ & $99.94 \%$ & 81403.3 \\
\hline YZ456 & YZ456 & $2021 / 8 / 10$ & $2021 / 8 / 11$ & $\mathrm{CI}$ & Moderate & NPS & YZ & multiplex-pcr & Illumina & 28 & 29 & 641,400 & $99.55 \%$ & 2597.14 \\
\hline YZ458 & YZ458 & 2021/8/9 & 2021/8/11 & $\mathrm{CI}$ & Mild & NPS & YZ & multiplex-pcr & Illumina & 18 & 20 & $1,880,124$ & $99.94 \%$ & 7588.175 \\
\hline YZ459 & YZ459 & $2021 / 8 / 10$ & 2021/8/12 & $\mathrm{CI}$ & Moderate & OPS & YZ & multiplex-pcr & Illumina & 0 & 33 & $3,311,557$ & $99.77 \%$ & 12476.32 \\
\hline YZ460 & YZ460 & $2021 / 8 / 10$ & $2021 / 8 / 11$ & $\mathrm{CI}$ & Mild & NPS & YZ & multiplex-pcr & Illumina & 29 & 28 & 802,102 & $99.57 \%$ & 3080.047 \\
\hline YZ461 & YZ461 & $2021 / 8 / 9$ & $2021 / 8 / 11$ & $\mathrm{CI}$ & Moderate & NPS & YZ & multiplex-pcr & Illumina & 31 & 27 & 388,093 & $99.48 \%$ & 1579.776 \\
\hline YZ462 & YZ462 & $2021 / 8 / 11$ & $2021 / 8 / 19$ & $\mathrm{CI}$ & Moderate & OPS & YZ & multiplex-pcr & Illumina & 28 & 29 & $8,306,466$ & $99.24 \%$ & 28682.06 \\
\hline YZ463 & YZ463 & $2021 / 8 / 11$ & $2021 / 8 / 11$ & $\mathrm{CI}$ & Moderate & NPS & YZ & multiplex-pcr & Illumina & 33 & 33 & 818,577 & $99.60 \%$ & 3196.083 \\
\hline YZ465 & YZ465 & $2021 / 8 / 5$ & $2021 / 8 / 12$ & II & Moderate & OPS & YZ & multiplex-pcr & BGI & 29 & 30 & $5,972,548$ & $99.90 \%$ & 19055.78 \\
\hline YZ466 & YZ466 & $2021 / 8 / 8$ & $2021 / 8 / 12$ & $\mathrm{CI}$ & Moderate & OPS & YZ & multiplex-pcr & BGI & 24 & 25 & $14,110,668$ & $99.94 \%$ & 44831.1 \\
\hline YZ467 & YZ467 & $2021 / 8 / 10$ & 2021/8/12 & CI & Mild & OPS & YZ & multiplex-pcr & BGI & 24 & 25 & $11,518,392$ & $99.94 \%$ & 36399.24 \\
\hline YZ468 & YZ468 & $2021 / 8 / 10$ & $2021 / 8 / 18$ & $\mathrm{CI}$ & Moderate & OPS & YZ & multiplex-pcr & Illumina & 23 & 24 & $10,467,360$ & $99.94 \%$ & 40308.7 \\
\hline YZ469 & YZ469 & $2021 / 8 / 10$ & $2021 / 8 / 12$ & $\mathrm{CI}$ & Mild & OPS & YZ & multiplex-pcr & Illumina & 28 & 27 & $1,136,282$ & $99.60 \%$ & 4600.826 \\
\hline YZ470 & YZ470 & $2021 / 8 / 10$ & 2021/8/12 & CI & Moderate & NPS & $\mathrm{YZ}$ & multiplex-pcr & Illumina & 31 & 30 & $1,097,410$ & $99.58 \%$ & 4439.936 \\
\hline YZ471 & YZ471 & $2021 / 8 / 10$ & 2021/8/12 & $\mathrm{CI}$ & Mild & NPS & YZ & multiplex-pcr & Illumina & 29 & 28 & $1,287,959$ & $99.45 \%$ & 5133.955 \\
\hline YZ472 & YZ472 & $2021 / 8 / 10$ & $2021 / 8 / 11$ & $\mathrm{CI}$ & Mild & OPS & YZ & multiplex-pcr & Illumina & 31 & 32 & $2,297,388$ & $99.78 \%$ & 9165.181 \\
\hline YZ473 & YZ473 & $2021 / 8 / 10$ & $2021 / 8 / 12$ & $\mathrm{CI}$ & Mild & OPS & YZ & multiplex-pcr & Illumina & 36 & 35 & $1,794,043$ & $99.86 \%$ & 7218.252 \\
\hline
\end{tabular}




\begin{tabular}{|c|c|c|c|c|c|c|c|c|c|c|c|c|c|c|}
\hline $\begin{array}{l}\mathrm{YZ} 475 \\
\end{array}$ & YZ475 & $2021 / 8 / 10$ & $2021 / 8 / 11$ & $\mathrm{CI}$ & Mild & OPS & YZ & multiplex-pcr & Illumina & 30 & 30 & 762,025 & $99.57 \%$ & 2980.38 \\
\hline YZ478 & YZ478 & $2021 / 8 / 7$ & $2021 / 8 / 11$ & II & Moderate & NPS & YZ & multiplex-pcr & Illumina & 22 & 22 & $24,686,913$ & $99.94 \%$ & 95555.85 \\
\hline YZ480 & YZ480 & $2021 / 8 / 10$ & $2021 / 8 / 12$ & $\mathrm{CI}$ & Moderate & OPS & YZ & multiplex-pcr & BGI & 22 & 24 & $13,775,207$ & $99.94 \%$ & 43777.45 \\
\hline YZ481 & YZ481 & $2021 / 8 / 10$ & $2021 / 8 / 12$ & $\mathrm{CI}$ & Severe & OPS & YZ & multiplex-pcr & BGI & 30 & 30 & 184,753 & $99.72 \%$ & 593.9336 \\
\hline YZ484 & YZ484 & $2021 / 8 / 11$ & $2021 / 8 / 18$ & $\mathrm{CI}$ & Moderate & OPS & YZ & multiplex-pcr & Illumina & 30 & 31 & $6,065,307$ & $99.72 \%$ & 23073.52 \\
\hline YZ485 & YZ485 & $2021 / 8 / 10$ & $2021 / 8 / 12$ & $\mathrm{CI}$ & Mild & NPS & YZ & multiplex-pcr & Illumina & 33 & 34 & $26,732,537$ & $99.93 \%$ & 105802.9 \\
\hline YZ486 & YZ486 & $2021 / 8 / 10$ & $2021 / 8 / 18$ & $\mathrm{CI}$ & Moderate & OPS & YZ & multiplex-pcr & Illumina & 25 & 25 & $6,361,492$ & $99.89 \%$ & 24114.53 \\
\hline YZ488 & YZ488 & $2021 / 8 / 10$ & $2021 / 8 / 18$ & $\mathrm{CI}$ & Moderate & OPS & YZ & multiplex-pcr & Illumina & 23 & 24 & $11,791,744$ & $99.94 \%$ & 44919.96 \\
\hline YZ489 & YZ489 & $2021 / 8 / 11$ & $2021 / 8 / 18$ & $\mathrm{CI}$ & Moderate & OPS & YZ & multiplex-pcr & Illumina & 25 & 25 & $6,090,301$ & $99.94 \%$ & 23121.42 \\
\hline YZ492 & YZ492 & $2021 / 8 / 10$ & 2021/8/12 & $\mathrm{CI}$ & Moderate & OPS & YZ & multiplex-pcr & Illumina & 30 & 30 & $2,829,372$ & $99.63 \%$ & 11461.95 \\
\hline YZ494 & YZ494 & $2021 / 8 / 9$ & $2021 / 8 / 12$ & $\mathrm{CI}$ & Moderate & OPS & YZ & multiplex-pcr & Illumina & 19 & 20 & $1,579,525$ & $99.61 \%$ & 6290.944 \\
\hline YZ497 & YZ497 & $2021 / 8 / 11$ & $2021 / 8 / 12$ & $\mathrm{CI}$ & Moderate & OPS & YZ & multiplex-pcr & Illumina & 20 & 21 & $1,100,006$ & $99.60 \%$ & 4457.015 \\
\hline YZ498 & YZ498 & $2021 / 8 / 6$ & $2021 / 8 / 18$ & II & Mild & OPS & YZ & multiplex-pcr & Illumina & 28 & 29 & $10,672,340$ & $99.93 \%$ & 41085.47 \\
\hline YZ499 & YZ499 & $2021 / 8 / 12$ & $2021 / 8 / 13$ & $\mathrm{CI}$ & Moderate & OPS & YZ & multiplex-pcr & Illumina & 26 & 27 & $1,679,771$ & $99.86 \%$ & 6713.408 \\
\hline YZ500 & YZ500 & 2021/8/11 & $2021 / 8 / 18$ & $\mathrm{CI}$ & Mild & OPS & YZ & multiplex-pcr & Illumina & 24 & 25 & $10,123,903$ & $99.91 \%$ & 38797.16 \\
\hline YZ504 & YZ504 & $2021 / 8 / 10$ & $2021 / 8 / 18$ & $\mathrm{CI}$ & Mild & OPS & YZ & multiplex-pcr & Illumina & 29 & 29 & $1,131,290$ & $99.45 \%$ & 3987.386 \\
\hline YZ505 & YZ505 & $2021 / 8 / 12$ & $2021 / 8 / 18$ & $\mathrm{CI}$ & Mild & OPS & YZ & multiplex-pcr & Illumina & 25 & 26 & 399,825 & $98.99 \%$ & 1495.327 \\
\hline YZ506 & YZ506 & $2021 / 8 / 11$ & $2021 / 8 / 13$ & $\mathrm{CI}$ & Mild & OPS & YZ & multiplex-pcr & Illumina & 25 & 32 & 358,592 & $99.02 \%$ & 1394.056 \\
\hline YZ507 & YZ507 & $2021 / 8 / 11$ & $2021 / 8 / 18$ & $\mathrm{CI}$ & Moderate & OPS & YZ & multiplex-pcr & Illumina & 32 & 33 & $4,194,722$ & $99.93 \%$ & 16049.52 \\
\hline YZ509 & YZ509 & $2021 / 8 / 10$ & $2021 / 8 / 18$ & $\mathrm{CI}$ & Mild & OPS & YZ & multiplex-pcr & Illumina & 32 & 33 & $2,897,692$ & $99.86 \%$ & 10708 \\
\hline YZ510 & YZ510 & $2021 / 8 / 10$ & $2021 / 8 / 18$ & $\mathrm{CI}$ & Moderate & OPS & YZ & multiplex-pcr & Illumina & 27 & 27 & $8,878,686$ & $99.94 \%$ & 33662.13 \\
\hline YZ512 & YZ512 & $2021 / 8 / 11$ & $2021 / 8 / 13$ & $\mathrm{CI}$ & Moderate & OPS & YZ & multiplex-pcr & Illumina & 23 & 27 & $1,423,963$ & $99.88 \%$ & 5715.885 \\
\hline YZ514 & YZ514 & $2021 / 8 / 12$ & $2021 / 8 / 18$ & $\mathrm{CI}$ & Moderate & OPS & YZ & multiplex-pcr & Illumina & 25 & 25 & $2,730,071$ & $99.64 \%$ & 10381.09 \\
\hline YZ515 & YZ515 & $2021 / 8 / 12$ & $2021 / 8 / 16$ & $\mathrm{CI}$ & Moderate & OPS & YZ & multiplex-pcr & Illumina & 31 & 34 & 701,680 & $99.75 \%$ & 2625.356 \\
\hline YZ516 & YZ516 & 2021/8/9 & $2021 / 8 / 16$ & $\mathrm{CI}$ & Moderate & OPS & YZ & multiplex-pcr & Illumina & 25 & 26 & $1,578,775$ & $99.83 \%$ & 6312.204 \\
\hline YZ517 & YZ517 & $2021 / 8 / 12$ & $2021 / 8 / 16$ & CI & Mild & OPS & $\mathrm{YZ}$ & multiplex-pcr & Illumina & 30 & 28 & $1,689,086$ & $99.84 \%$ & 6751.423 \\
\hline YZ518 & YZ518 & 2021/8/12 & $2021 / 8 / 18$ & $\mathrm{CI}$ & Moderate & NPS & YZ & multiplex-pcr & Illumina & 18 & 19 & $10,403,785$ & $99.93 \%$ & 36565.65 \\
\hline YZ519 & YZ519 & $2021 / 8 / 12$ & $2021 / 8 / 18$ & $\mathrm{CI}$ & Moderate & OPS & YZ & multiplex-pcr & Illumina & 23 & 24 & $1,383,281$ & $99.88 \%$ & 4847.055 \\
\hline YZ522 & YZ522 & $2021 / 8 / 12$ & $2021 / 8 / 13$ & $\mathrm{CI}$ & Moderate & OPS & YZ & multiplex-pcr & Illumina & 23 & 24 & 980,260 & $99.76 \%$ & 3782.601 \\
\hline
\end{tabular}




\begin{tabular}{|c|c|c|c|c|c|c|c|c|c|c|c|c|c|c|}
\hline YZ523 & YZ523 & $2021 / 8 / 12$ & $2021 / 8 / 18$ & $\mathrm{CI}$ & Moderate & OPS & YZ & multiplex-pcr & Illumina & 28 & 29 & 889,943 & $99.59 \%$ & 3267.013 \\
\hline YZ524 & YZ524 & $2021 / 8 / 13$ & $2021 / 8 / 14$ & $\mathrm{CI}$ & Moderate & OPS & YZ & multiplex-pcr & Illumina & 24 & 25 & $1,437,113$ & $99.90 \%$ & 5781.894 \\
\hline YZ525 & YZ525 & $2021 / 8 / 12$ & $2021 / 8 / 18$ & $\mathrm{CI}$ & Moderate & OPS & YZ & multiplex-pcr & Illumina & 19 & 20 & $11,534,996$ & $99.91 \%$ & 44240.5 \\
\hline YZ526 & YZ526 & $2021 / 8 / 10$ & $2021 / 8 / 18$ & $\mathrm{CI}$ & Moderate & OPS & YZ & multiplex-pcr & Illumina & 29 & 30 & $15,000,466$ & $99.94 \%$ & 55482.26 \\
\hline YZ531 & YZ531 & $2021 / 8 / 13$ & $2021 / 8 / 18$ & CI & Moderate & NPS & YZ & multiplex-pcr & Illumina & 17 & 18 & $10,735,438$ & $99.94 \%$ & 40623.74 \\
\hline YZ532 & YZ532 & $2021 / 8 / 13$ & $2021 / 8 / 15$ & CI & Moderate & NPS & YZ & multiplex-pcr & Illumina & 16 & 18 & $1,704,304$ & $99.55 \%$ & 6373.416 \\
\hline YZ534 & YZ534 & $2021 / 8 / 13$ & $2021 / 8 / 18$ & $\mathrm{CI}$ & Moderate & NPS & YZ & multiplex-pcr & Illumina & 22 & 23 & $4,891,887$ & $99.93 \%$ & 18559.2 \\
\hline YZ535 & YZ535 & $2021 / 8 / 13$ & $2021 / 8 / 15$ & $\mathrm{CI}$ & Moderate & OPS & YZ & multiplex-pcr & Illumina & 31 & 27 & 508,379 & $99.58 \%$ & 2033.586 \\
\hline YZ536 & YZ536 & $2021 / 8 / 14$ & $2021 / 8 / 18$ & $\mathrm{CI}$ & Moderate & NPS & YZ & multiplex-pcr & Illumina & 19 & 20 & $26,301,042$ & $99.94 \%$ & 99605.61 \\
\hline YZ537 & YZ537 & 2021/8/13 & $2021 / 8 / 18$ & $\mathrm{CI}$ & Moderate & OPS & YZ & multiplex-pcr & Illumina & 21 & 22 & $11,139,714$ & $99.94 \%$ & 42842.83 \\
\hline YZ539 & YZ539 & $2021 / 8 / 11$ & $2021 / 8 / 18$ & $\mathrm{CI}$ & Moderate & NPS & YZ & multiplex-pcr & Illumina & 27 & 27 & $13,756,097$ & $99.92 \%$ & 51334.64 \\
\hline YZ540 & YZ540 & $2021 / 8 / 4$ & $2021 / 8 / 14$ & $\mathrm{CI}$ & Moderate & OPS & YZ & multiplex-pcr & Illumina & 23 & 24 & 790,282 & $99.82 \%$ & 3174.38 \\
\hline YZ541 & YZ541 & 2021/8/13 & $2021 / 8 / 18$ & $\mathrm{CI}$ & Moderate & OPS & YZ & multiplex-pcr & Illumina & 30 & 30 & $8,803,186$ & $99.94 \%$ & 33754.01 \\
\hline YZ542 & YZ542 & $2021 / 8 / 13$ & $2021 / 8 / 18$ & $\mathrm{CI}$ & Moderate & OPS & YZ & multiplex-pcr & Illumina & 19 & 20 & $6,817,804$ & $99.91 \%$ & 25319.28 \\
\hline YZ545 & YZ545 & 2021/8/13 & $2021 / 8 / 18$ & CI & Moderate & NPS & YZ & multiplex-pcr & Illumina & 26 & 27 & $12,309,648$ & $99.94 \%$ & 47530.96 \\
\hline YZ546 & YZ546 & 2021/8/13 & 2021/8/15 & $\mathrm{CI}$ & Moderate & OPS & YZ & multiplex-pcr & Illumina & 30 & 30 & 165,921 & $99.70 \%$ & 668.936 \\
\hline YZ547 & YZ547 & $2021 / 8 / 14$ & $2021 / 8 / 18$ & $\mathrm{CI}$ & Mild & OPS & YZ & multiplex-pcr & Illumina & 31 & 32 & $11,897,197$ & $99.94 \%$ & 45605.08 \\
\hline YZ548 & YZ548 & $2021 / 8 / 13$ & $2021 / 8 / 18$ & $\mathrm{CI}$ & Moderate & NPS & YZ & multiplex-pcr & Illumina & 18 & 19 & $11,881,278$ & $99.94 \%$ & 45815.42 \\
\hline YZ549 & YZ549 & $2021 / 8 / 10$ & $2021 / 8 / 15$ & CI & Moderate & NPS & YZ & multiplex-pcr & Illumina & 33 & 31 & 385,667 & $99.41 \%$ & 1536.305 \\
\hline YZ551 & YZ551 & $2021 / 8 / 15$ & $2021 / 8 / 19$ & $\mathrm{CI}$ & Mild & NPS & YZ & multiplex-pcr & Illumina & 29 & 30 & $12,026,776$ & $99.91 \%$ & 45284.34 \\
\hline YZ552 & YZ552 & $2021 / 8 / 15$ & $2021 / 8 / 18$ & $\mathrm{CI}$ & Mild & NPS & YZ & multiplex-pcr & Illumina & 22 & 23 & $1,599,325$ & $99.63 \%$ & 5909.415 \\
\hline YZ553 & YZ553 & $2021 / 8 / 15$ & $2021 / 8 / 18$ & $\mathrm{CI}$ & Moderate & NPS & YZ & multiplex-pcr & Illumina & 27 & 28 & $12,219,751$ & $99.94 \%$ & 46040.41 \\
\hline YZ554 & YZ554 & $2021 / 8 / 5$ & 2021/8/17 & II & Moderate & OPS & YZ & multiplex-pcr & Illumina & 29 & 30 & $1,866,221$ & $99.92 \%$ & 7289.246 \\
\hline YZ559 & YZ559 & $2021 / 8 / 16$ & $2021 / 8 / 17$ & ER & Moderate & OPS & YZ & multiplex-pcr & Illumina & 22 & 24 & $2,026,302$ & $99.92 \%$ & 7550.223 \\
\hline YZ560 & YZ560 & $2021 / 8 / 16$ & $2021 / 8 / 16$ & ER & Mild & OPS & YZ & multiplex-pcr & Illumina & 33 & 33 & 779,946 & $99.89 \%$ & 2702.612 \\
\hline YZ561 & YZ561 & $2021 / 8 / 16$ & $2021 / 8 / 18$ & ER & Mild & OPS & $\mathrm{YZ}$ & multiplex-pcr & Illumina & 32 & 33 & $6,410,587$ & $99.85 \%$ & 25273.11 \\
\hline YZ562 & YZ562 & $2021 / 8 / 10$ & $2021 / 8 / 18$ & CI & Moderate & OPS & YZ & multiplex-pcr & Illumina & 29 & 30 & $6,127,909$ & $99.85 \%$ & 24298.09 \\
\hline YZ564 & YZ564 & $2021 / 8 / 16$ & $2021 / 8 / 18$ & ER & Moderate & OPS & YZ & multiplex-pcr & Illumina & 29 & 31 & $5,532,590$ & $99.90 \%$ & 21918.26 \\
\hline YZ570 & YZ570 & $2021 / 8 / 25$ & $2021 / 8 / 18$ & ER & Mild & OPS & YZ & multiplex-pcr & Illumina & 28 & 29 & $6,392,719$ & $99.71 \%$ & 22901.38 \\
\hline
\end{tabular}




\begin{tabular}{|c|c|c|c|c|c|c|c|}
\hline \multirow[t]{16}{*}{$\mathrm{NJ} / \mathrm{Yu}$} & $2021 / 7 / 10$ & $2021 / 7 / 10$ & NA & Asympt & OPS & multiplex-pcr & Illumina \\
\hline & $\mathrm{XB}$ & $2021 / 5 / 14$ & & & & Jiangsu import & \\
\hline & XSG & $2021 / 6 / 13$ & & & & Jiangsu import & \\
\hline & GSF & $2021 / 6 / 17$ & & & & Jiangsu import & \\
\hline & $\mathrm{ZYC}$ & $2021 / 6 / 22$ & & & & Jiangsu import & \\
\hline & CXJ & $2021 / 6 / 22$ & & & & Jiangsu import & \\
\hline & $\mathrm{BN}$ & $2021 / 6 / 22$ & & & & Jiangsu import & \\
\hline & IVDC-06-01 & $2021 / 6 / 6$ & & & & GD & \\
\hline & IVDC-0610-39 & $2021 / 6 / 25$ & & & & GD & \\
\hline & IVDC-0610-01 & $2021 / 6 / 11$ & & & & GD & \\
\hline & IVDC-05-01 & $2021 / 4 / 17$ & & & & GD & \\
\hline & Ruili-IVDC-04-01 & $2021 / 3 / 31$ & & & & YN & \\
\hline & Ruili-IVDC-04-02 & $2021 / 3 / 30$ & & & & YN & \\
\hline & $\mathrm{YN}-01$ & $2021 / 6 / 17$ & & & & $\mathrm{YN}$ & \\
\hline & YN-47 & $2021 / 7 / 8$ & & & & $\mathrm{YN}$ & \\
\hline & DL-ILBS-22053 & $2020 / 6 / 13$ & & & & New Delhi & \\
\hline
\end{tabular}

